\title{
GEOMETRIC CONSTANT TERM FUNCTOR(S)
}

\author{
V. DRINFELD AND D. GAITSGORY \\ To Joseph Bernstein with deepest gratitude
}

\begin{abstract}
We study the Eisenstein series and constant term functors in the framework of geometric theory of automorphic functions. Our main result says that for a parabolic $P \subset G$ with Levi quotient $M$, the !-constant term functor $\mathrm{CT}_{!}: \mathrm{D}-\bmod \left(\operatorname{Bun}_{G}\right) \rightarrow \mathrm{D}-\bmod \left(\operatorname{Bun}_{M}\right)$

is canonically isomorphic to the ${ }^{*}$-constant term functor $\mathrm{CT}_{*}^{-}: \mathrm{D}-\bmod \left(\operatorname{Bun}_{G}\right) \rightarrow \mathrm{D}-\bmod \left(\operatorname{Bun}_{M}\right)$, taken with respect to the opposite parabolic $P^{-}$.
\end{abstract}

\section{INTRODUCTION}

0.1. Conventions. We fix an algebraically closed field $k$ of characteristic 0. Unless stated otherwise, we say "scheme" instead of "scheme locally of finite type over $k$." When we say "stack" we mean an algebraic stack locally of finite type over $k$ such that the automorphism group of any $k$-point is affine. 1

A morphism of stacks $f: Y_{1} \rightarrow Y_{2}$ is said to be representable (resp. schematic) if for any scheme $S$ the stack $Y_{1} \underset{Y_{2}}{\times} S$ is an algebraic space (resp. scheme).

\subsection{Posing the problem.}

0.2.1. One of the main tools in studying automorphic functions on an adèle group $G$ is the pair of mutually adjoint operators, called "Eisenstein series" and "constant term" that connect this space to similar spaces for Levi subgroups.

The goal of this paper is to make several basic observations regarding the analogs of these operators in the geometric context.

0.2.2. Let $X$ be a smooth projective connected curve over $k$, and $G$ a reductive group. Let $\operatorname{Bun}_{G}$ be the moduli stack of principal $G$-bundles on $X$. Our geometric analog of the space of automorphic functions is the DG category of (not necessarily holonomic) D-modules on $\operatorname{Bun}_{G}$, denoted by D-mod $\left(\operatorname{Bun}_{G}\right)$. We refer the reader to Sect. 0.6.2 for the explanation of what exactly we understand by "DG category of D-modules." Here we just mention that the homotopy category of this DG category is the derived category of D-modules.

\footnotetext{
Date: October 6, 2016.

${ }^{1}$ The latter condition is called "locally QCA" in DrGa1. It ensures that the theory of D-modules on our stack is reasonable.
} 
Let $P$ be a parabolic in $G$ with Levi quotient $M$. We have the following fundamental diagram of stacks:

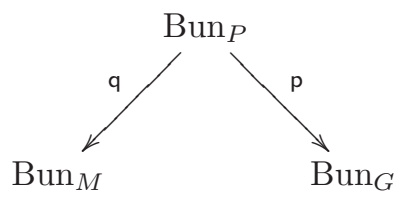

Recall that the set of connected components of the stack Bun $\operatorname{Bu}_{M}$ is in bijection with $\pi_{1}(M)$. For an element $\mu \in \pi_{1}(M)$ we let $\operatorname{Bun}_{M}^{\mu}$ denote the corresponding connected component; let $\operatorname{Bun}_{P}^{\mu}$ denote the preimage of $\operatorname{Bun}_{M}^{\mu} \subset \operatorname{Bun}_{M}$ under the above map q : $\operatorname{Bun}_{P} \rightarrow \operatorname{Bun}_{M}$; one can show that $\operatorname{Bun}_{P}^{\mu}$ is connected, i.e., is a single connected component of $\mathrm{Bun}_{P}$.

By a slight abuse of notation we will denote by the same symbols q and $\mathrm{p}$, respectively, the restrictions of the corresponding maps to $\operatorname{Bun}_{P}^{\mu} \subset \operatorname{Bun}_{P}$. Thus, we obtain a diagram

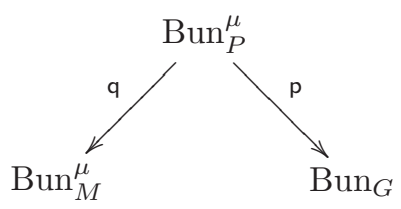

0.2.3. Applying pull-push along diagram (0.2) we obtain the functors

$$
\operatorname{Eis}_{*}^{\mu}: \mathrm{D}-\bmod \left(\operatorname{Bun}_{M}^{\mu}\right) \rightarrow \mathrm{D}-\bmod \left(\operatorname{Bun}_{G}\right), \quad \operatorname{Eis}_{*}^{\mu}:=\mathrm{p}_{*} \circ \mathrm{q}^{!}
$$

and

$$
\mathrm{CT}_{*}^{\mu}: \mathrm{D}-\bmod \left(\operatorname{Bun}_{G}\right) \rightarrow \mathrm{D}-\bmod \left(\operatorname{Bun}_{M}^{\mu}\right), \quad \mathrm{CT}_{*}^{\mu}:=\mathrm{q}_{*} \circ \mathrm{p} !
$$

(here Eis stands for "Eisenstein" and CT stands for "constant term").

We will address the following questions:

Question 0.2.4. Can one define the functor

$$
\operatorname{Eis}_{!}^{\mu}:=\mathrm{p}_{!} \circ \mathrm{q}^{*}: \mathrm{D}-\bmod \left(\operatorname{Bun}_{M}^{\mu}\right) \rightarrow \mathrm{D}-\bmod \left(\operatorname{Bun}_{G}\right),
$$

left adjoint to $\mathrm{CT}_{*}^{\mu}$ ?

Question 0.2.5. Can one define the functor

$$
\mathrm{CT}_{!}^{\mu}:=\mathrm{q} ! \circ \mathrm{p}^{*}: \mathrm{D}-\bmod \left(\operatorname{Bun}_{G}\right) \rightarrow \mathrm{D}-\bmod \left(\operatorname{Bun}_{M}^{\mu}\right),
$$

left adjoint to $\operatorname{Eis}_{*}^{\mu}$ ?

The next few remarks explain why these questions are non-obvious.

Remark 0.2.6. For a morphism $f: Y_{1} \rightarrow Y_{2}$ between schemes or stacks the functors

$$
f_{*}: \mathrm{D}-\bmod \left(Y_{1}\right) \rightarrow \mathrm{D}-\bmod \left(D_{2}\right) \text { and } f^{!}: \mathrm{D}-\bmod \left(Y_{1}\right) \rightarrow \mathrm{D}-\bmod \left(D_{2}\right)
$$

are well-defined. But their left adjoints

$$
f^{*}: \mathrm{D}-\bmod \left(Y_{2}\right) \rightarrow \mathrm{D}-\bmod \left(D_{1}\right) \text { and } f_{!}: \mathrm{D}-\bmod \left(Y_{2}\right) \rightarrow \mathrm{D}-\bmod \left(D_{1}\right)
$$

are only partially defined (because we work with non-necessarily holonomic D-modules).

Remark 0.2.7. If $f$ is quasi-compact, representable and proper then $f !$ is always defined and equals $f_{*}$. If $f$ is smooth then $f^{*}$ is always defined and equals $f^{!}$up to a cohomological shift. 
Remark 0.2.8. If a morphism of stacks $f: Y_{1} \rightarrow Y_{2}$ is safe then the functor $f_{*}$ is "well-behaved" (more precisely, $f_{*}$ is continuous). For more details (including the definitions of safety and continuity), see Sect. 0.6.3.

Remark 0.2.9. In diagram (0.2) the morphism $\mathrm{p}$ is quasi-compact and representable (and moreover, schematic), but neither smooth nor proper. The morphism q is smooth and safe 2 (but not representable).

\section{3 . Statement of the results.}

0.3.1. First, we show that the functor $\operatorname{Eis}_{!}^{\mu}:=\mathrm{p}_{!} \circ \mathrm{q}^{*}$ is well-defined. (More precisely, since $\mathrm{q}$ is smooth the functor $q^{*}$ is well-defined, and we will show that $p_{\text {! }}$ is well-defined on the essential image of $\mathrm{q}^{*}$ ).

0.3.2. We now turn to $\mathrm{CT}_{!}^{\mu}$. We do eventually show that the functor $\mathrm{CT}_{!}^{\mu}$ is well-defined, but this comes as a result of a more precise assertion (see Theorem 0.3 .4 below) that describes this functor explicitly.

Here we note that a direct attempt to define $\mathrm{CT}_{1}^{\mu}=\mathrm{q}$ ! $\circ \mathrm{p}^{*}$ brings problems different from those in the case of $\operatorname{Eis}_{1}^{\mu}$. Namely, it turns out that the second functor, i.e., q!, is always welldefined (see Sect. 4.1.6), while the first one, namely p*, is not. However, we will show that their composition is well-defined in a certain sense (see Sect. 4.3 .2 for the general paradigm when such compositions are well-defined; we will show that this paradigm is applicable to $\mathrm{Bun}_{G}$ in Sect. 4.7 and Sect. 4.1.6).

0.3.3. Now we are ready to state the main result of this paper, namely, Theorem 1.2 .3

Theorem 0.3.4. The functor $\mathrm{CT}_{!}^{\mu}$ exists and is isomorphic to the functor $\mathrm{CT}_{*}^{\mu,-}$, where the superscript "-" means that instead of $P$ we are considering the opposite parabolic $P^{-}$.

The assertion of this theorem can be viewed as some kind of non-standard functional equation. It does not have an immediate analog in the classical theory of automorphic functions (where one has only one type of pullback operator and one type of push forward).

Theorem 0.3.4 has an implication to the relation between the functors Eis! and Eis ${ }_{*}$, which is discussed in [Ga, Theorem 4.1.2]. Here we will only mention that this implication does have a manifestation in the classical theory of automorphic functions.

0.4. Relation to the geometric Langlands conjecture. The contents of this subsection play a motivational role and may be skipped by the reader.

0.4.1. In addition to the functors

$$
\operatorname{Eis}_{*}^{\mu}, \quad \mathrm{CT}_{*}^{\mu}, \quad \mathrm{Eis}_{!}^{\mu}, \quad \mathrm{CT}_{!}^{\mu}
$$

one can consider the full Eisenstein and constant term functors:

$$
\operatorname{Eis}_{*}:=\underset{\mu \in \pi_{1}(M)}{\oplus} \operatorname{Eis}_{*}^{\mu}, \quad \text { Eis! }:=\underset{\mu \in \pi_{1}(M)}{\oplus} \operatorname{Eis}_{!}^{\mu}
$$

and

$$
\begin{aligned}
& \mathrm{CT}_{*}:=\underset{\mu \in \pi_{1}(M)}{\oplus} \mathrm{CT}_{*}^{\mu} \simeq \prod_{\mu \in \pi_{1}(M)} \mathrm{CT}_{*}^{\mu}, \\
& \mathrm{CT}_{!}:=\underset{\mu \in \pi_{1}(M)}{\oplus} \mathrm{CT}_{!}^{\mu} \simeq \prod_{\mu \in \pi_{1}(M)} \mathrm{CT}_{!}^{\mu},
\end{aligned}
$$

\footnotetext{
${ }^{2}$ For any surjection of algebraic groups $\phi: H_{1} \rightarrow H_{2}$ the corresponding map $\Phi: \operatorname{Bun}_{H_{1}} \rightarrow$ Bun $_{H_{2}}$ is smooth, which can be seen through the calculation of its differential. If $\operatorname{ker}(\phi)$ is unipotent, then $\Phi$ is safe.
} 
(where the sum equals the product because each $\mathrm{CT}_{*}^{\mu}$ and $\mathrm{CT}_{!}^{\mu}$ lands in its own component of $\left.\operatorname{Bun}_{M}^{\mu}\right)$.

Tautologically, the functors (Eis! $\mathrm{CT}_{*}$ ) form an adjoint pair.

Note, however, that the functors $\mathrm{CT}_{\text {! }}$ and $\mathrm{Eis}_{*}$ do not form an adjoint pair. Indeed, the right adjoint of $\mathrm{CT}_{\text {! }}$ is given by $\underset{\mu \in \pi_{1}(M)}{\prod_{*}}$ Eis $_{*}^{\mu}$ (which is different from $\operatorname{Eis}_{*}:=\underset{\mu \in \pi_{1}(M)}{\oplus} \operatorname{Eis}_{*}^{\mu}$ ); in particular, the above right adjoint is not continuous.

Define also

$$
\mathrm{CT}_{*}^{-}:=\underset{\mu \in \pi_{1}(M)}{\oplus} \mathrm{CT}_{*}^{\mu,-} .
$$

Of course, Theorem 0.3 .4 implies that we have a canonical isomorphism

$$
\mathrm{CT}_{!} \simeq \mathrm{CT}_{*}^{-} .
$$

0.4.2. It turns out that the functor Eis!, although less straightforward to define than Eis $_{*}$, plays a more fundamental role from the point of the geometric Langlands conjecture.

Namely, according to $\mathrm{AG}$, this conjecture predicts an equivalence of categories

$$
\mathbb{L}_{G}: \mathrm{D}-\bmod \left(\operatorname{Bun}_{G}\right) \rightarrow \operatorname{IndCoh}_{\mathrm{Nil}_{\text {glob }}}\left(\operatorname{LocSys}_{\breve{G}}\right),
$$

where $\operatorname{IndCoh}_{\mathrm{Nil}_{\text {glob }}}\left(\operatorname{LocSys}_{\breve{G}}\right)$ is a certain modification of the DG category of quasi-coherent sheaves on the stack LocSys $\breve{G}$ of local systems on $X$ with respect to the Langands dual group $\check{G}$.

Now, the equivalence $\mathbb{L}_{G}$ is expected to be compatible with the corresponding equivalence $\mathbb{L}_{M}$ for its Levi subgroup $M$ via the diagram

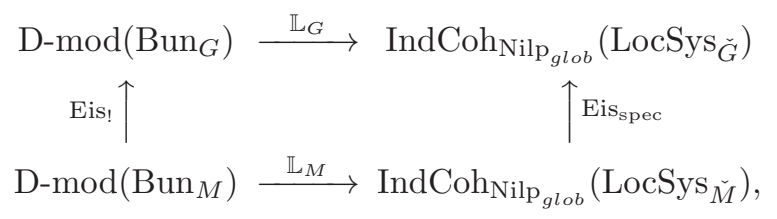

which commutes up to an auto-equivalence of $\operatorname{IndCoh}_{\mathrm{Nil}_{\text {glob }}}\left(\operatorname{LocSys}_{\check{M}}\right)$, given by tensoring with a certain canonically defined graded line bundle on $\operatorname{LocSys}_{\check{M}}$.

In the above diagram, Eis $_{\text {spec }}$ is the spectral Eisenstein series functor, defined as pull-push along the diagram

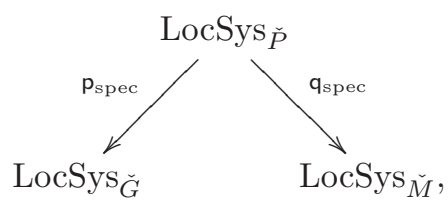

see [AG, Sect. 13.2] for more details.

The point that we would like to emphasize is that the commutation in diagram (0.3) takes place for the functor Eis! and not for Eis $_{*}$ (moreover, this cannot be remedied by any autoequivalence of the category D-mod( $\left(\operatorname{Bun}_{G}\right)$ 3.

It is possible to explicitly describe the functor

$$
\operatorname{IndCoh}_{\mathrm{Nilp}_{\text {glob }}}\left(\operatorname{LocSys}_{\check{M}}\right) \rightarrow \operatorname{IndCoh}_{\mathrm{Nilp}_{\text {glob }}}\left(\operatorname{LocSys}_{\check{G}}\right)
$$

\footnotetext{
${ }^{3}$ See, however, Sect. 0.4 .4 below.
} 
that corresponds via $\mathbb{L}_{G}$ and $\mathbb{L}_{M}$ to

$$
\operatorname{Eis}_{*}: \mathrm{D}-\bmod \left(\operatorname{Bun}_{M}\right) \rightarrow \mathrm{D}-\bmod \left(\operatorname{Bun}_{G}\right),
$$

but this description is more involved.

0.4.3. The fact that the functor Eis! is more fundamental than Eis $*$ can also be explained as follows.

Recall (see DrGa2, Sect. 4.3.3]) that in addition to the category D-mod(Bun $\left.{ }_{G}\right)$, there exists another DG category, denoted $\mathrm{D}-\bmod \left(\mathrm{Bun}_{G}\right)_{\mathrm{co}}$, that can be naturally assigned to the stack $\operatorname{Bun}_{G}$.

Furthermore, according to [DrGa2, Sect. 4.4.3], there is a canonically defined functor

$$
\text { Ps- } \operatorname{Id}_{\text {Bun }_{G}, \text { naive }}: \mathrm{D}-\bmod \left(\operatorname{Bun}_{G}\right)_{\text {co }} \rightarrow \mathrm{D}-\bmod \left(\operatorname{Bun}_{G}\right),
$$

which is not an equivalence, unless $G$ is a torus.

Now, it is not difficult to see that there is a naturally defined functor

$$
\operatorname{Eis}_{*, \text { co }}: \mathrm{D}-\bmod \left(\operatorname{Bun}_{M}\right)_{\text {co }} \rightarrow \mathrm{D}-\bmod \left(\operatorname{Bun}_{G}\right)_{\text {co }}
$$

that makes the following diagram commute:

$$
\begin{aligned}
& \mathrm{D}-\bmod \left(\operatorname{Bun}_{G}\right)_{\text {co }} \stackrel{\text { Ps-Id }_{\text {Bun }_{G}, \text { naive }}}{\longrightarrow} \mathrm{D}-\bmod \left(\operatorname{Bun}_{G}\right) \\
& \mathrm{Eis}_{*, \mathrm{co}} \uparrow \quad \mathrm{Eis}_{*} \uparrow
\end{aligned}
$$

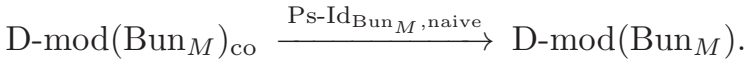

Thus, the functor $\mathrm{Eis}_{*}$ is a coarsening of $\mathrm{Eis}_{*, \text { co }}$, since, informally, the functor Ps- $\operatorname{Id}_{\mathrm{Bun}_{G}, \text { naive }}$ "loses more information" than Ps-Id $\mathrm{Bun}_{M}$, naive.

0.4.4. Finally, we note that according to [DrGa2, Sect. 4.4.8], in addition to the functor $\mathrm{PS}_{\mathrm{S}-\mathrm{Id}_{\mathrm{Bun}}, \text { naive }}$, there is another canonically defined functor

$$
\text { Ps- } \operatorname{Id}_{\mathrm{Bun}_{G}, !}: \mathrm{D}-\bmod \left(\operatorname{Bun}_{G}\right)_{\mathrm{co}} \rightarrow \mathrm{D}-\bmod \left(\operatorname{Bun}_{G}\right) \text {. }
$$

It is shown in [Ga, Theorem 3.1.5] that the functor Ps- $\mathrm{Id}_{\mathrm{Bun}_{G}, !}$ is actually an equivalence of categories.

In $\mathrm{Ga}$, Theorem 4.1.2] it is also shown that the following diagram commutes:

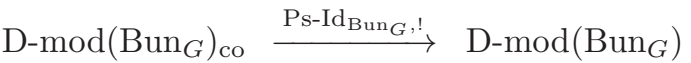

$$
\begin{aligned}
& \mathrm{Eis}_{*, \mathrm{co}} \uparrow \quad \mathrm{Eis}_{!}^{-} \uparrow
\end{aligned}
$$

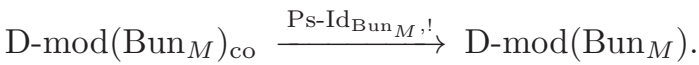

So, to summarize, although the naive functor Eis $*$ plays an inferior role to that of Eis! its counterpart

$$
\operatorname{Eis}_{*, \text { co }}: \mathrm{D}-\bmod \left(\operatorname{Bun}_{M}\right)_{\text {co }} \rightarrow \mathrm{D}-\bmod \left(\operatorname{Bun}_{G}\right)_{\text {co }}
$$

is on par with Eis! by virtue of being intertwined by the equivalences Ps- $\operatorname{Id}_{\mathrm{Bun}_{G}, !}$ and Ps-Id $\operatorname{Bun}_{M}, ! \cdot$

In [Ga, Sect. 0.2], it is also explained how diagram (0.5) expresses the compatibility of the Langlands correspondence functors $\mathbb{L}_{G}\left(\right.$ resp., $\left.\mathbb{L}_{M}\right)$ with Verdier duality on Bun $\operatorname{Bu}_{G}\left(\right.$ resp., $\left.\operatorname{Bun}_{M}\right)$ and Serre duality on $\operatorname{LocSys}_{\breve{G}}\left(\operatorname{resp}, \operatorname{LocSys}_{\check{M}}\right)$. 
0.5. Method of proof. The proof of Theorem 0.3 .4 is a variation on the theme of a theorem of T. Braden on hyperbolic restrictions (see $\mathrm{Br}$ ), recently revisited in DrGa3]. In fact, we give two proofs, in Sects. $2+3$ and Sect. 4 respectively.

0.5.1. The first proof mimics the new proof of Braden's theorem given in DrGa3, and it directly establishes the $\left(\mathrm{CT}_{*}^{\mu,-}\right.$, $\left.\mathrm{Eis}_{*}^{\mu}\right)$-adjunction by specifying the unit and co-unit morphisms.

As in the case of the new proof of Braden's theorem given in DrGa3, the co-unit morphism is straightforward, and essentially corresponds to the embedding of the big Bruhat cell into $P \backslash G / P^{-}$.

0.5.2. The unit of the adjunction uses a certain geometric construction, namely, an $\mathbb{A}^{1}$-family of subgroups $\widetilde{G}$ of $G \times G$, whose fiber at $1 \in \mathbb{A}^{1}$ is the diagonal copy of $G$, and whose fiber at $0 \in \mathbb{A}^{1}$ is the subgroup

$$
P \underset{M}{\times} P^{-} \subset P \times P^{-} \subset G \times G .
$$

For the definition of the group-subscheme $\widetilde{G} \subset \mathbb{A}^{1} \times G \times G$, see Sects. 2.3.2, 2.3.3, and 2.4, Here let us just mention that $\widetilde{G}$ depends on the choice of a co-character

$$
\gamma: \mathbb{G}_{m} \rightarrow M
$$

which lands in the center of $M$, and which is dominant and regular with respect to $P$.

0.5.3. The group-scheme $\widetilde{G}$ is not new in Lie theory. Namely, it can be recovered from the Vinberg semi-group correspondingg to $G$ (a.k.a. the enveloping semi-group of $G$ ); see Appendix D. where this is explained.

The Lie algebra $\operatorname{Lie}(\widetilde{G})$ can be directly recovered from the "wonderful compactification" of $G$ defined in $\mathrm{DCP}$ :

Let $W$ denote the variety of Lie subalgebras of $\mathfrak{g} \times \mathfrak{g}$, where $\mathfrak{g}:=\operatorname{Lie}(G)$. Let $\gamma: \mathbb{G}_{m} \rightarrow M$ denote the co-character (0.6). For $t \in \mathbb{G}_{m}$ let $\Gamma_{t} \subset \mathfrak{g} \times \mathfrak{g}$ denoted the graph of $\operatorname{Ad}_{\mu(t)}: \mathfrak{g} \stackrel{\sim}{\longrightarrow} \mathfrak{g}$. Since $W$ is projective the map $\mathbb{G}_{m} \rightarrow W$ defined by $t \mapsto \Gamma_{t}$ extends to a morphism $\mathbb{A}^{1} \rightarrow W$, whose image is contained in the "wonderful compactification." Thus one gets an $\mathbb{A}^{1}$-family of Lie subalgebras of $\mathfrak{g} \times \mathfrak{g}$. This $\mathbb{A}^{1}$-family is $\operatorname{Lie}(\widetilde{G})$ (because $\widetilde{G}$ is smooth over $\mathbb{A}^{1}$, see Proposition 2.3.8).

0.5.4. The statement that the functors $\left(\mathrm{CT}_{*}^{\mu,-}, \mathrm{Eis}_{*}^{\mu}\right)$ form an adjoint pair bears a strong resemblance to the Second Adjointness Theorem in the theory of $\mathfrak{p}$-adic groups.

By a slight abuse of notation, let us temporarily denote by $G$ the set of points of a reductive group over a local non-archimedian field, and consider the corresponding groups

$$
M \nleftarrow P \hookrightarrow G .
$$

We have the usual pair of adjoint functors

$$
\left(r_{M}^{G}, i_{M}^{G}\right)
$$

where $i_{M}^{G}$ is the functor of parabolic induction, and $r_{M}^{G}$ is the Jacquet functor.

Now, a theorem of J. Bernstein (unpublished) says that, in addition to being the right adjoint of $r_{M}^{G}$, the functor $i_{M}^{G}$ is also the left adjoint of $r_{M}^{G,-}$, where the latter is the Jacquet functor with respect to the opposite parabolic $P^{-}$.

The proof of this result, given recently in $\mathrm{BKa}$ (which is different from the original proof of Bernstein), is very close in spirit to our proof of the $\left(\mathrm{CT}_{*}^{\mu,-}, \mathrm{Eis}_{*}^{\mu}\right)$-adjunction: 
One constructs the unit of the adjunction using the big Bruhat cell in $N(P) \backslash G / N\left(P^{-}\right)$.

The co-unit of the adjunction uses (the set of points over our field of) the family of schemes over $\mathbb{A}^{1}$

$$
\left(\mathbb{A}^{1} \times G \times G\right) / \widetilde{G}
$$

acted on by $G \times G$. Note that this family interpolates between $G$ (the fiber at $1 \in \mathbb{A}^{1}$ ) and $\left(G / N(P) \times G / N\left(P^{-}\right)\right) / M$ (the fiber at $\left.0 \in \mathbb{A}^{1}\right)$.

0.5.5. Remark. The proof of Braden's theorem given in DrGa3 is paraphrased in Dr, Sect. C.14] using the categorical formalism of "lax actions by correspondences". This formalism can also be used to prove Theorem 0.3.4. However, we will not use it in this article.

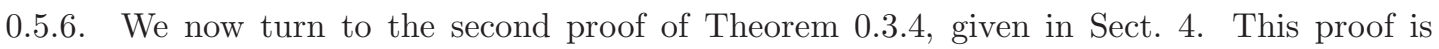
obtained by deducing the isomorphism

$$
\mathrm{CT}_{!}^{\mu} \simeq \mathrm{CT}_{*}^{\mu,-}
$$

from Braden's theorem, which involves schemes acted on by $\mathbb{G}_{m}$.

The schemes in question are obtained by replacing $\operatorname{Bun}_{G}, \operatorname{Bun}_{P}, \operatorname{Bun}_{P-}$ and $\operatorname{Bun}_{M}$ by their versions when one considers a sufficiently deep level structure at one point of the curve.

0.5.7. Finally, we remark that Theorem 0.3 .4 is analogous to the corresponding theorem of Lusztig on restriction of character sheaves, see [Gi, Theorem 4.1 (iv)].

In fact, the two statements admit a common generalization when instead of $\mathrm{Bun}_{G}$ we consider the moduli stack of $G$-bundles with level structure at a finite collection of points of $X$; the case of character sheaves on $G$ (resp., the Lie algebra $\mathfrak{g}$ ) corresponds to the case of $X=\mathbb{P}^{1}$ with structure of level 1 at $(0, \infty) \in \mathbb{P}^{1}$ (resp., structure of level 2 ar $\infty \in \mathbb{P}^{1}$ ).

Just as Theorem 0.3.4 Lusztig's theorem can be deduced from Braden's theorem. We learned this very simple proof of Lusztig's theorem from folklore and wrote it up in DrGa3, Sect. 0.2].

\subsection{Recollections on D-modules on stacks.}

0.6.1. DG categories. Our conventions and notation pertaining to DG categories follow those of [DrGa2, Sect. 1].

0.6.2. The category $\mathrm{D}-\bmod (\mathrm{y})$, where $y$ is a stack. For any stack $y$ let $\mathrm{D}$-mod(y) denote the DG category of D-modules on $y$ as defined in DrGa1 (the case of quasi-compact schemes is considered in [DrGa1, Sect. 5.1] and the general case in [DrGa1, Sect. 6.2]).

If $y=S$ is a quasi-compact scheme then the homotopy category of the DG category $\mathrm{D}-\bmod (S)$ is the usual derived category of D-modules on $S$, but note that we impose no boundedness or coherence conditions 4 .

If $y$ is a stack then $\mathrm{D}-\bmod (y)$ is defined to be the (projective) limit of the categories D-mod $(S)$ over the indexing category of quasi-compact schemes $S$ mapping smoothly to $y$. Informally, an object of $\mathrm{D}-\bmod (\mathrm{y})$ is a compatible collection of objects of $\mathrm{D}-\bmod (S)$ for all quasi-compact schemes $S$ mapping smoothly to $y$.

As is explained in DrGa1, the DG category D-mod(y) is cocomplete, i.e., it has arbitrary colimits.

\footnotetext{
${ }^{4}$ This is because we want $\mathrm{D}-\bmod (S)$ to be cocomplete, see below.
} 
0.6.3. Direct images and safety. For any morphism $f: y_{1} \rightarrow y_{2}$ between stacks one has the direct image functor $f_{*}: D-\bmod \left(y_{1}\right) \rightarrow \mathrm{D}-\bmod \left(y_{2}\right)$ (see [DrGa1, Sect. 7.4] for the definition of $f_{*}$ in the case that $f$ is not necessarily quasi-compact schematic).

The functor $f_{*}$ is not necessarily continuous. (Recall that a functor between cocomplete DG categories is said to be continuous if it commutes with (infinite) colimits, or equivalently, with (infinite) direct sums.)

However, according to DrGa1, Sect. 10.2], $f_{*}$ is continuous if $f$ is safe. By definition, safety means that $f$ is quasi-compact and has the following property: for any $y \in y_{2}(k)$ the neutral connected component of the automorphism group of any $k$-point of the fiber $\left(y_{1}\right)_{y}$ is unipotent. In particular, any quasi-compact representable morphism is safe.

0.6.4. Terminological remark. Following the conventions of higher category theory, we call a morphism between two objects in a DG category an isomorphism if and only if it is such in the homotopy category.

0.7. Organization of the article. In Sect. 1 we reformulate Theorem 0.3 .4 as an existence of an adjunction (see Theorem 1.2.5), and we describe the natural transformation that will turn out to be the co-unit of the adjunction. We also discuss the notion of cuspidal object of $\mathcal{F} \in \mathrm{D}-\bmod \left(\operatorname{Bun}_{G}\right)$; the main point is that two a priori different notions of cuspidality coincide.

As already said in 0.5 , we give two proofs of Theorem 1.2 .5

The first one is given in Sections 2] (In Sect. 2] we construct the natural transformation that will turn out to be the unit, and in Sect. 3 we verify that the natural transformations constructed in Sections 1 and 2 satisfy the properties of unit and count of an adjunction.)

The second proof of Theorem 1.2.5 is given in Sect. 固

In Appendix $\mathrm{A}$ we prove a technical Theorem 4.3.4, which is used in the second proof of Theorem 1.2.5.

In Appendix B we prove Proposition 1.4.6 that describes support of cusipdal objects of $\mathrm{D}-\bmod \left(\operatorname{Bun}_{G}\right)$.

In Appendix $\mathbb{C}$ we prove Proposition 2.4.4, which says that the quotient of $\mathbb{A}^{1} \times G \times G$ by the "interpolating" group-subscheme $\widetilde{G} \subset \mathbb{A}^{1} \times G \times G$ is a quasi-affine scheme.

In Appendix $\mathbb{D}$ we describe $\widetilde{G}$ and $\left(\mathbb{A}^{1} \times G \times G\right) / \widetilde{G}$ in terms of the Vinberg semigroup of $G$ (a.k.a. enveloping semigroup of $G$ ).

0.8. Acknowledgements. The research of V. D. is partially supported by NSF grants DMS1001660 and DMS-1303100. The research of D. G. is partially supported by NSF grant DMS1063470.

\section{The Statement}

\subsection{The functor $\mathrm{Eis}_{!}^{\mu}$.}


1.1.1. Consider the diagram

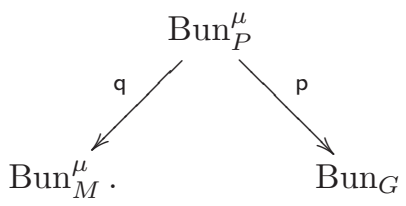

By Remark 0.2.9 the map $\mathrm{q}$ is smooth, so the functor $\mathrm{q}^{*}$, left adjoint to $\mathrm{q}_{*}$, is well-defined. We are going to prove:

Proposition 1.1.2. The partially defined left adjoint $\mathrm{p}_{\text {! }}$ to $\mathrm{p}$ ! is defined on the essential image of the functor $\mathrm{q}^{*}$.

Corollary 1.1.3. The functor $\operatorname{Eis}_{!}^{\mu}:=\mathrm{p}_{!} \circ \mathrm{q}^{*}: \mathrm{D}-\bmod \left(\operatorname{Bun}_{M}^{\mu}\right) \rightarrow \mathrm{D}-\bmod \left(\operatorname{Bun}_{G}\right)$, left adjoint to $\mathrm{CT}_{*}^{\mu}$, is well-defined.

The proof of Proposition 1.1.2 given below, is based on some results of [BG]. Let us recall them.

1.1.4. First, the diagram (1.1) was extended in BG, Sect. 1.2] to a diagram

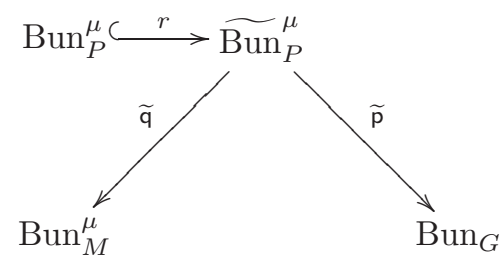

so that $\widetilde{p}$ is proper, $r$ is an open embedding, and

$$
\mathrm{p}=\widetilde{\mathrm{p}} \circ r, \quad \mathrm{q}=\widetilde{\mathrm{q}} \circ r .
$$

Moreover, the following basic fact was established (see [BG, Theorem 5.1.5]):

The object $r_{!}\left(k_{\mathrm{Bun}_{P}^{\mu}}\right) \in \mathrm{D}-\bmod \left(\widetilde{\operatorname{Bun}_{P}}\right)$ is universally locally acyclic (ULA) with respect to the map $\widetilde{\mathrm{q}}$. Here for a stack $y$, we denote by $k_{y} \in \mathrm{D}$ - $\bmod (y)$ the "constant sheaf" D-module on $y$ (i.e., the Verdier dual of $\omega y$ ).

1.1.5. Let us recall the definition of the ULA property. First, recall that on D-mod(y) there are two tensor products, namely

$$
\mathcal{F}_{1} \stackrel{!}{\otimes} \mathcal{F}_{2}:=\Delta ! \dot{y}\left(\mathcal{F}_{1} \otimes \mathcal{F}_{2}\right) \quad \text { and } \quad \mathcal{F}_{1} \stackrel{*}{\otimes} \mathcal{F}_{2}:=\Delta_{y}^{*}\left(\mathcal{F}_{1} \otimes \mathcal{F}_{2}\right), \quad \mathcal{F}_{1}, \mathcal{F}_{2} \in \operatorname{D}-\bmod (y),
$$

where $\Delta y: y \rightarrow y \times y$ is the diagonal morphism. (Note that $\Delta^{*}$ and $\stackrel{*}{\otimes}$ are only partially defined.)

Suppose now that we have a morphism $\phi: y \rightarrow z$ with $z$ smooth. According to BG, Sect. 5.1.1], $\mathcal{F} \in \mathrm{D}-\bmod (y)$ is said to be $U L A$ with respect to $\phi$ if for every $\mathcal{F}^{\prime} \in \mathrm{D}-\bmod (z)$ the following holds: $\mathcal{F}^{*} \otimes \phi^{*}\left(\mathcal{F}^{\prime}\right)$ is a well-defined object of $\mathrm{D}-\bmod (y)$ and a certain canonical morphism

$$
\mathcal{F}^{*} \otimes \phi^{*}\left(\mathcal{F}^{\prime}\right) \rightarrow \mathcal{F} \stackrel{!}{\otimes} \phi^{!}\left(\mathcal{F}^{\prime}\right)[2 \operatorname{dim}(Z)],
$$

defined in [BG, Sect. 5.1.1], is an isomorphism. 
1.1.6. Proof of Proposition 1.1.2. Since $\mathrm{p}=\widetilde{\mathrm{p}} \circ r$ and the functor $\widetilde{\mathrm{p}}_{!}=\widetilde{\mathrm{p}}_{*}$ is well-defined, it suffices to show that $r_{!} \circ \mathrm{q}^{*}(\mathcal{F})$ is well-defined for every $\mathcal{F} \in \mathrm{D}-\bmod \left(\operatorname{Bun}_{M}^{\mu}\right)$.

This follows from the ULA property of $r_{!}\left(k_{\mathrm{Bun}_{P}^{\mu}}\right)$ because $r ! \circ \mathrm{q}^{*}(\mathcal{F})=r_{!}\left(k_{\mathrm{Bun}_{P}^{\mu}}\right) \stackrel{\otimes}{\otimes} \widetilde{\mathrm{q}}^{*}(\mathcal{F})$.

1.2. The functor $\mathrm{CT}_{!}^{\mu}$. Our next task is to analyze the existence of the left adjoint of the functor $\operatorname{Eis}_{*}$.

1.2.1. Let $P^{-}$be a parabolic opposite to $P$. We will identify the Levi factors of $P$ and $P^{-}$ via the embedding of $M \simeq P \cap P^{-}$into $P$ and $P^{-}$, respectively.

In particular, we have the diagram

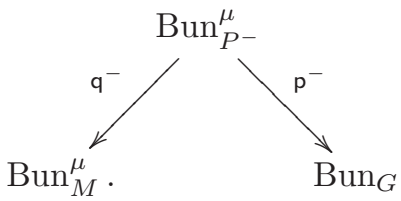

Let $\mathrm{Eis}_{*}^{\mu,-}$, $\mathrm{Eis}_{!}^{\mu,-}$ and $\mathrm{CT}_{*}^{\mu,-}$ be the counterparts of $\mathrm{Eis}_{*}^{\mu}, \mathrm{Eis}_{!}^{\mu}$ and $\mathrm{CT}_{*}^{\mu}$, obtained via the diagram (1.3).

1.2.2. We are now ready to formulate our main result:

Theorem 1.2.3. The functor

$$
\mathrm{CT}_{!}^{\mu}: \mathrm{D}-\bmod \left(\operatorname{Bun}_{G}\right) \rightarrow \mathrm{D}-\bmod \left(\operatorname{Bun}_{M}^{\mu}\right),
$$

left adjoint to $\mathrm{Eis}_{*}^{\mu}$, exists and is canonically isomorphic to $\mathrm{CT}_{*}^{\mu,-}$.

Thus we have a sequence of three functors

$$
\mathrm{Eis}_{!}^{\mu,-}, \quad \mathrm{CT}_{*}^{\mu,-}=\mathrm{CT}_{!}^{\mu}, \quad \operatorname{Eis}_{*}^{\mu}
$$

in which each neighboring pair forms an adjoint pair of functors.

1.2.4. Theorem 1.2 .3 can be tautologically restated as follows:

Theorem 1.2.5. The functors $\left(\mathrm{CT}_{*}^{\mu,-}, \mathrm{Eis}_{*}^{\mu}\right)$ form an adjoint pair.

In Sect. 2. we will prove Theorem 1.2.5 by essentially repeating the argument from the paper DrGa3] that gives a new proof of a theorem of T. Braden (see $[\mathrm{Br}$ ) on hyperbolic restrictions.

In Sect. 4 we will give a proof of Theorem 1.2 .5 by directly deducing it from the above theorem of Braden.

\subsection{Description of the co-unit of the adjunction.}


1.3.1. Let us specify the co-unit of the adjunction for the functors $\left(\mathrm{CT}_{*}^{\mu,-}, \mathrm{Eis}_{*}^{\mu}\right)$ in Theorem 1.2.5, i.e., the natural transformation

$$
\mathrm{CT}_{*}^{\mu,-} \circ \operatorname{Eis}_{*}^{\mu} \rightarrow \mathrm{Id}_{\mathrm{D}-\bmod \left(\operatorname{Bun}_{M}^{\mu}\right)} \cdot
$$

Consider the diagram

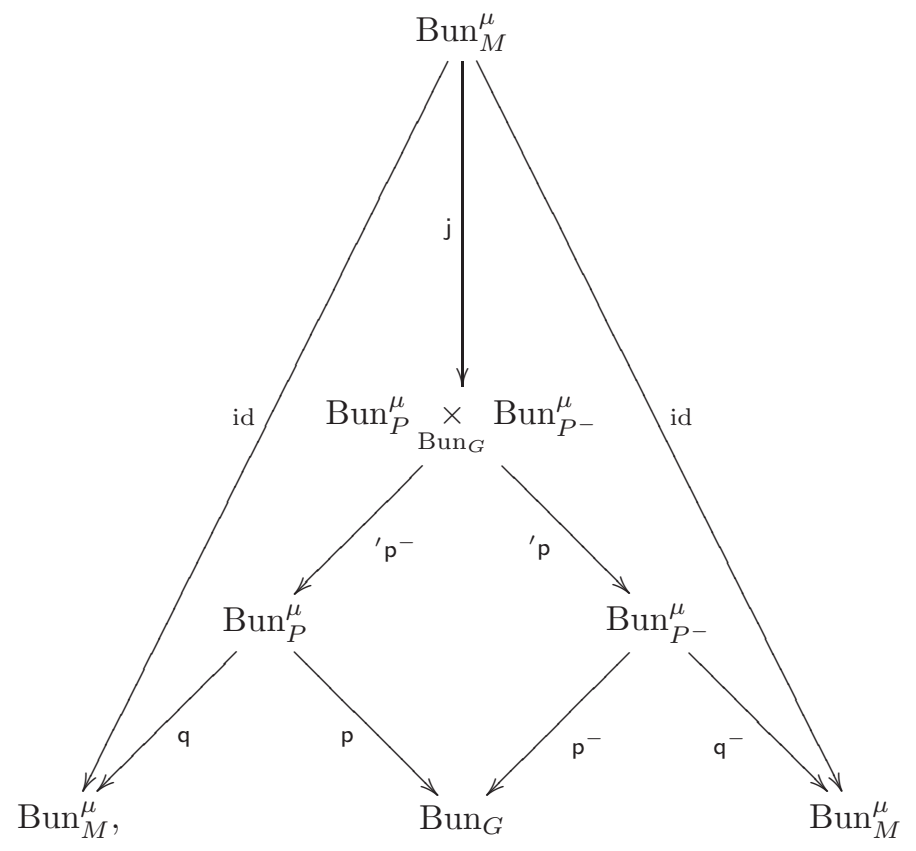

where $\mathrm{j}$ is the (open) embedding of the big Bruhat cell, i.e., the locus where the reductions of a given $G$-bundle to $P$ and $P^{-}$are mutually transversal 5

By base change,

$$
\mathrm{CT}_{*}^{\mu,-} \circ \operatorname{Eis}_{*}^{\mu}=\left(\mathrm{q}^{-}\right)_{*} \circ\left(\mathrm{p}^{-}\right)^{!} \circ \mathrm{p}_{*} \circ \mathrm{q}^{!} \simeq\left(\mathrm{q}^{-}\right)_{*} \circ\left({ }^{\prime} \mathrm{p}\right)_{*} \circ\left({ }^{\prime} \mathrm{p}^{-}\right)^{!} \circ \mathrm{q}^{!} .
$$

Now, the open embedding $\mathrm{j}$ gives rise to a natural transformation

$$
\operatorname{Id}_{\mathrm{D}-\bmod \left(\operatorname{Bun}_{P}^{\mu} \underset{\text { Bun }_{G}}{\times} \operatorname{Bun}_{P^{-}}^{\mu}\right)} \rightarrow \mathrm{j}_{*} \circ \mathrm{j}^{*} \simeq \mathrm{j}_{*} \circ \mathrm{j}^{!},
$$

and hence to

$$
\begin{aligned}
& \mathrm{CT}_{*}^{\mu,-} \circ \operatorname{Eis}_{*}^{\mu} \simeq\left(\mathrm{q}^{-}\right)_{*} \circ\left({ }^{\prime} \mathrm{p}\right)_{*} \circ\left({ }^{\prime} \mathrm{p}^{-}\right)^{!} \circ \mathrm{q}^{!} \rightarrow \\
& \rightarrow\left(\mathrm{q}^{-}\right)_{*} \circ\left({ }^{\prime} \mathrm{p}\right)_{*} \circ \mathrm{j}_{*} \circ \mathrm{j}^{!} \circ\left({ }^{\prime} \mathrm{p}^{-}\right)^{!} \circ \mathrm{q}^{!} \simeq \\
& \simeq\left(\mathrm{q}^{-} \circ \circ^{\prime} \mathrm{p} \circ \mathrm{j}\right)_{*} \circ\left(\mathrm{q} \circ \mathrm{p}^{-} \circ \mathrm{j}\right)^{!} \simeq \mathrm{id}_{*} \circ \mathrm{id} !^{!}=\operatorname{Id}_{\mathrm{D}-\bmod \left(\operatorname{Bun}_{M}^{\mu}\right) .}
\end{aligned}
$$

This is the sought-for co-unit of the adjuction.

\footnotetext{
${ }^{5}$ The morphism $\mathrm{j}$ is of the type considered in Lemma 2.5.8 (ii) below.
} 
1.3.2. Let us observe that Theorem 1.2 .3 can be reformulated also as saying that the functors $\left(\mathrm{Eis}_{!}^{\mu,-}, \mathrm{CT}_{!}^{\mu}\right)$ form an adjoint pair. The above description of the co-unit for the $\left(\mathrm{CT}_{*}^{\mu,-}, \mathrm{Eis}_{*}^{\mu}\right)-$ adjunction gives rise to a description of the unit of the $\left(\mathrm{Eis}_{!}^{\mu,-}, \mathrm{CT}_{!}^{\mu}\right)$-adjunction:

By definition, the functors $\mathrm{Eis}_{!}^{\mu,-}$ and $\mathrm{CT}_{!}^{\mu}$ are the left adjoints of the functors $\mathrm{CT}_{*}^{\mu,-}$ and $\operatorname{Eis}_{*}^{\mu}$, respectively. Now, the unit map

$$
\mathrm{Id}_{\mathrm{D}-\bmod \left(\operatorname{Bun}_{M}^{\mu}\right)} \rightarrow \mathrm{CT}_{!}^{\mu} \circ \mathrm{Eis}_{!}^{\mu,-}
$$

is obtained by passing to left adjoints in the map (1.4).

\subsection{Cuspidality.}

1.4.1. We will say that an object $\mathcal{F} \in \mathrm{D}$ - $\bmod \left(\mathrm{Bun}_{G}\right)$ is ${ }^{*}$-cuspidal if $\mathrm{CT}_{*}^{\mu}(\mathcal{F})=0$ for all proper parabolics of $G$.

We will say that an object $\mathcal{F} \in \mathrm{D}-\bmod \left(\mathrm{Bun}_{G}\right)$ is !-cuspidal $\mathrm{CT}_{!}^{\mu}(\mathcal{F})=0$ for all proper parabolics of $G$.

1.4.2. From Theorem 1.2 .3 we obtain:

Corollary 1.4.3. The notions of !- and ${ }^{*}$-cuspidality coincide.

Hence, from now on, we will rename the above property as just cuspidality. Let $\mathrm{D}-\bmod \left(\operatorname{Bun}_{G}\right)_{\text {cusp }}$ be the full subcategory of $\mathrm{D}-\bmod \left(\operatorname{Bun}_{G}\right)$ formed by cuspidal objects. By construction, D- $\bmod \left(\operatorname{Bun}_{G}\right)_{\text {cusp }}$ is cocomplete (i.e., closed under colimits).

Let us denote by $\mathrm{D}-\bmod \left(\operatorname{Bun}_{G}\right)_{\mathrm{Eis}, !}\left(\right.$ resp., $\left.\mathrm{D}-\bmod \left(\operatorname{Bun}_{G}\right)_{\mathrm{Eis}, *}\right)$ the full subcategory of $\mathrm{D}-\bmod \left(\operatorname{Bun}_{G}\right)$ generated 6 by the essential images of the functors $\operatorname{Eis}_{!}^{\mu}$ (resp., Eis $_{*}^{\mu}$ ) for all proper parabolics.

Viewing the notion of cuspidality from the ${ }^{*}$-perspective, we have:

$$
\mathrm{D}-\bmod \left(\operatorname{Bun}_{G}\right)_{\text {cusp }}=\left(\mathrm{D}-\bmod \left(\operatorname{Bun}_{G}\right)_{\text {Eis, } !}\right)^{\perp} .
$$

1.4.4. In addition, we have the inclusion

$$
{ }^{\perp}\left(\mathrm{D}-\bmod \left(\operatorname{Bun}_{G}\right)_{\text {Eis }, *}\right) \subset \mathrm{D}-\bmod \left(\operatorname{Bun}_{G}\right)_{\text {cusp }}
$$

that comes from

$$
{ }^{\perp}\left(\mathrm{D}-\bmod \left(\operatorname{Bun}_{G}\right)_{\text {Eis }, *}\right) \subset \underset{P, \mu}{\cap}\left(\operatorname{Im}\left(\operatorname{Eis}_{*}^{\mu}\right)\right)=\underset{P, \mu}{\cap} \operatorname{ker}\left(\mathrm{CT}_{!}^{\mu}\right)=\mathrm{D}-\bmod \left(\operatorname{Bun}_{G}\right)_{\text {cusp }} .
$$

It is not clear whether the inclusion (1.5) is an equality; we conjecture that it is not if $X$ has genus $\geq 2$.

1.4.5. Let us note another important property of cuspidal objects:

Proposition 1.4.6. There exists an open substack $\jmath: \mathcal{U} \hookrightarrow \mathrm{Bun}_{G}$ such that

(i) The intersection of $\mathfrak{U}$ with each connected component of $\mathrm{Bun}_{G}$ is quasi-compact;

(ii) For any $\mathcal{F} \in \mathrm{D}-\bmod \left(\operatorname{Bun}_{G}\right)_{\text {cusp }}$, the canonical maps

$$
\jmath ! \circ \jmath^{*}(\mathcal{F}) \rightarrow \mathcal{F} \rightarrow \jmath_{*} \circ \jmath^{*}(\mathcal{F})
$$

are isomorphisms 7

\footnotetext{
6 "generated by $A$ " means "the smallest cocomplete DG subcategory, containing $A$ "

${ }^{7}$ The statement about $\jmath ! \circ \jmath^{*}(\mathcal{F})$ should be understood as follows: the partially defined functor $\jmath !$ is defined on $\jmath^{*}(\mathcal{F})$ and the map $\jmath ! \circ \jmath^{*}(\mathcal{F}) \rightarrow \mathcal{F}$ is an isomorphism.
} 
The proof is given in Appendix B. It is parallel to the proof of a similar statement in the classical theory of automorphic forms. In fact, in Appendix B we describe an explicit open substack $\mathcal{U}$ with the properties required in Proposition 1.4.6.

\section{Proof of Theorem 1.2.5. Constructing the unit of the adjunction}

We will prove Theorem 1.2.5 (and thereby Theorem 1.2.3) by mimicking the new proof of Braden's theorem given in DrGa3.

The idea of the proof is to define a natural transformation

$$
\mathrm{Id}_{\mathrm{D}-\bmod \left(\operatorname{Bun}_{G}\right)} \rightarrow \operatorname{Eis}_{*}^{\mu} \circ \mathrm{CT}_{*}^{\mu,-}
$$

and to show that (1.4) and (2.1) define an adjunction datum.

In this section we will construct the natural transformation (2.1). The fact that (1.4) and (2.1) indeed define an adjunction datum will be proved in Sect. 3

2.1. Digression: functors given by kernels. As was mentioned in Sect. 0.1 all algebraic stacks in this paper are assumed locally QCA.

2.1.1. Let $f: y_{1} \rightarrow y_{2}$ be a quasi-compact map. We note that in addition to the usual de Rham direct image functor

$$
f_{*}: \mathrm{D}-\bmod \left(y_{1}\right) \rightarrow \mathrm{D}-\bmod \left(y_{2}\right)
$$

(defined as in DrGa1, Sect. 7.4]), there exists another canonically defined functor

$$
f_{\mathbf{\Delta}}: \mathrm{D}-\bmod \left(y_{1}\right) \rightarrow \mathrm{D}-\bmod \left(y_{2}\right) \text {. }
$$

The main feature of the functor $f_{\mathbf{\Delta}}$ is that, unlike $f_{*}$, it is continuous. In addition, it has the following properties (all of which fail for $f_{*}$ ):

(i) The formation $f \rightsquigarrow f_{\mathbf{\Delta}}$ is compatible with compositions of morphisms;

(ii) It satisfies base change (with respect to !-pullbacks);

(iii) It satisfies the projection formula.

We have a natural transformation

$$
f_{\mathbf{\Delta}} \rightarrow f_{*} .
$$

If the morphism $f$ is representable, or, more generally, safe, then (2.2) is an isomorphism.

All of the above facts are established in DrGa1, Sect. 9.3].

Remark 2.1.2. Technically, in DrGa1, Sect. 9.3], the functor $f_{\boldsymbol{\Delta}}$ was defined when $y_{2}$ (and, hence, $\left.y_{1}\right)$ is quasi-compact. However, the base-change property ensures that the definition canonically extends to the case of general quasi-compact morphisms.

2.1.3. Let $y_{1}$ and $y_{2}$ be two algebraic stacks. We will say that an object

$$
Q \in \mathrm{D}-\bmod \left(y_{1} \times y_{2}\right)
$$

has a quasi-compact !-support relative to $y_{2}$ if the following condition holds:

For every quasi-compact open substack $U_{2} \stackrel{j_{2}}{\hookrightarrow} y_{2}$ there exists a quasi-compact open substack $U_{1} \stackrel{j_{1}}{\longrightarrow} y_{1}$ such that the map

$$
\left(\mathrm{id} \times j_{2}\right)^{*}(Q) \rightarrow\left(j_{1} \times \mathrm{id}\right)_{*} \circ\left(j_{1} \times \mathrm{id}\right)^{*} \circ\left(\mathrm{id} \times j_{2}\right)^{*}(\mathrm{Q})=\left(j_{1} \times \mathrm{id}\right)_{*} \circ\left(j_{1} \times j_{2}\right)^{*}(\mathrm{Q})
$$

is an isomorphism in $\mathrm{D}-\bmod \left(y_{1} \times U_{2}\right)$. 
Let us repeat the same in words: the restriction of $\mathcal{Q}$ to the open substack $y_{1} \times U_{2}$ is a *-extension from $U_{1} \times U_{2}$ for some quasi-compact open substack $U_{1} \subset y_{1}$.

Note that $\left(j_{1} \times \mathrm{id}\right)_{*} \simeq\left(j_{1} \times \mathrm{id}\right)_{\boldsymbol{\Delta}}$ (indeed, the morphism $j_{1} \times$ id is representable because it is an open embedding).

2.1.4. Let $\mathcal{Q} \in \mathrm{D}$ - $\bmod \left(y_{1} \times y_{2}\right)$ have a quasi-compact !-support relative to $y_{2}$. We claim that such $\mathcal{Q}$ canonically gives rise to a continuous functor

$$
\mathrm{F}_{\mathcal{Q}}: \mathrm{D}-\bmod \left(y_{1}\right) \rightarrow \mathrm{D}-\bmod \left(y_{2}\right), \quad \mathrm{F}_{\mathcal{Q}}(\mathcal{F}):=\left(\operatorname{pr}_{2}\right)_{\Delta}\left(\operatorname{pr}_{1}^{*}(\mathcal{F}) \stackrel{!}{\otimes} \mathcal{Q}\right) .
$$

Since the morphism $\mathrm{pr}_{2}$ is not assumed quasi-compact, we have to explain how to understand formula (2.4).

By the definition of D-mod( $\left(y_{2}\right)$ (see DrGa2, Sect. 2.3] for a detailed review), the datum of a functor $D-\bmod \left(y_{1}\right) \rightarrow D-\bmod \left(y_{2}\right)$ amounts to a compatible 8 family of functors

$$
\mathrm{D}-\bmod \left(y_{1}\right) \rightarrow \mathrm{D}-\bmod \left(U_{2}\right)
$$

for quasi-compact open substacks $U_{2} \stackrel{j_{2}}{\longrightarrow} y_{2}$.

For a given $U_{2}$, let $U_{1} \stackrel{j_{1}}{\longrightarrow} y_{1}$ be as in Sect. 2.1.3, and let

$$
\operatorname{pr}_{2}^{U_{1}, U_{2}}: U_{1} \times U_{2} \rightarrow U_{2}
$$

denote the projection. Then we set

$$
j_{2}^{*}\left(\mathrm{~F}_{\mathcal{Q}}(\mathcal{F})\right):=\left(\operatorname{pr}_{2}^{U_{1}, U_{2}}\right)_{\mathbf{\Delta}} \circ\left(j_{1} \times j_{2}\right)^{*}\left(\operatorname{pr}_{1}^{*}(\mathcal{F}) \stackrel{!}{\otimes} \mathcal{Q}\right)
$$

(here the $\boldsymbol{\Lambda}$-puhsforward is defined because the morphism $\operatorname{pr}_{2}^{U_{1}, U_{2}}$ is quasi-compact).

It is easy to see that the isomorphism (2.3) implies that the right-hand side in (2.5) is independent of the choice of $U_{1}$.

Furthermore, it is easy to see that the base change property for the $\mathbf{\Delta}$-puhsforward for quasicompact morphisms implies that the functors (2.5) are indeed compatible under the inclusions $U_{2}^{\prime} \subset U_{2}^{\prime \prime}$.

2.1.5. As an example, consider a diagram

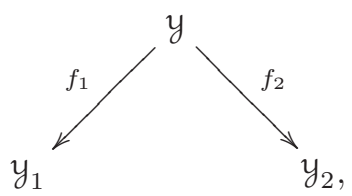

where the morphism $f_{2}$ is quasi-compact. In this case the morphism

$$
\left(f_{1} \times f_{2}\right): y \rightarrow y_{1} \times y_{2}
$$

is quasi-compact as well.

Set

$$
\mathcal{Q}:=\left(f_{1} \times f_{2}\right) \boldsymbol{\Delta}(\omega y) \in \mathrm{D}-\bmod \left(y_{1} \times y_{2}\right),
$$

where $\omega_{y} \in \mathrm{D}-\bmod (y)$ is the dualizing complex.

It is easy to see that $\mathcal{Q}$ has a quasi-compact !-support relative to $y_{2}$. Moreover, in this case the functor $\mathrm{F}_{Q}$ identifies canonically with $\left(f_{2}\right)_{\mathbf{\Delta}} \circ f_{1}^{*}$.

\footnotetext{
${ }^{8}$ The compatibility is with respect to ${ }^{*}$-restrictions for the inclusions $U_{2}^{\prime} \subset U_{2}^{\prime \prime}$.
} 
In particular, for $y_{1}=y=y_{2}$ and $f_{1}=f_{2}=i d$, we obtain that for $\mathcal{Q}:=(\Delta y) \Delta(\omega y)$, the corresponding functor $\mathrm{F}_{\mathcal{Q}}$ identifies canonically with $\operatorname{Id}_{\mathrm{D}-\bmod (y)}$.

\subsection{Plan of the construction.}

2.2.1. Note that since the morphism $\mathrm{p}$ is quasi-compact and representable (in fact, schematic), we have:

$$
\operatorname{Eis}_{*}^{\mu}=\mathrm{p}_{*} \circ \mathrm{q}^{!} \simeq \mathrm{p}_{\mathbf{\Delta}} \circ \mathrm{q}^{!} .
$$

Since q is safe, we have also

$$
\mathrm{CT}_{*}^{\mu}=\mathrm{q}_{*} \circ \mathrm{p}^{!} \simeq \mathrm{q}_{\mathbf{\Delta}} \circ \mathrm{p}^{!}
$$

Hence, the functor $\mathrm{Eis}_{*}^{\mu} \circ \mathrm{CT}_{*}^{\mu,-}$ is given as pull-push along the following diagram:

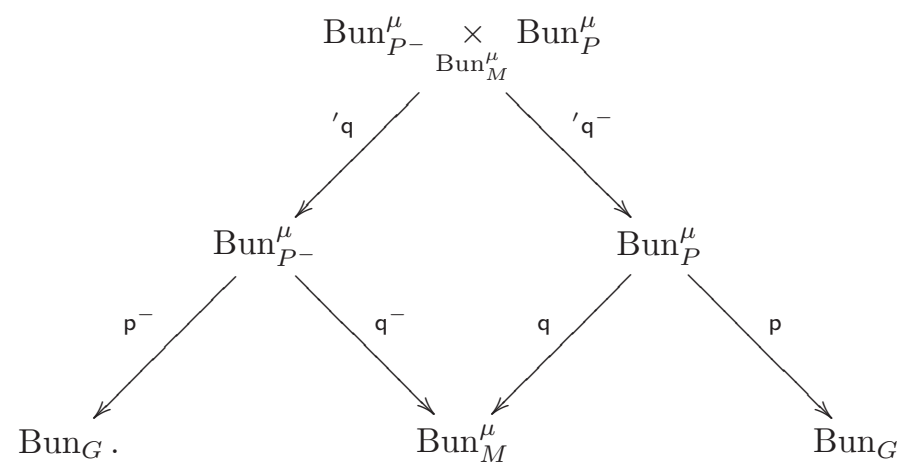

Here and in the sequel, by "push" we understand the $\boldsymbol{\Delta}$-pushforward, so the base change formula applies to any quasi-compact morphism.

2.2.2. Denote

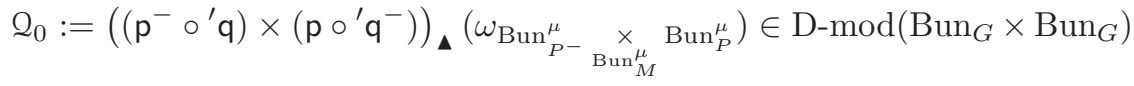

and

$$
Q_{1}:=\left(\Delta_{\operatorname{Bun}_{G}}\right) \mathbf{\Delta}\left(\omega_{\mathrm{Bun}_{G}}\right) \in \mathrm{D}-\bmod \left(\operatorname{Bun}_{G} \times \operatorname{Bun}_{G}\right) .
$$

By Sect. 2.1.5 we have:

Lemma 2.2.3. The functors $\mathrm{Id}_{\mathrm{D}-\bmod \left(\mathrm{Bun}_{G}\right)}$ and $\mathrm{Eis}_{*}^{\mu} \circ \mathrm{CT}_{*}^{\mu,-}$ are given by $\mathrm{F}_{Q_{1}}$ and $\mathrm{F}_{Q_{0}}$, respectively.

2.2.4. We are going to define the natural transformation (2.1) by constructing a map

$$
Q_{1} \rightarrow Q_{0}
$$

in D-mod $\left(\operatorname{Bun}_{G} \times \operatorname{Bun}_{G}\right)$. To do this, we will construct an object

$$
\mathcal{Q} \in \mathrm{D}-\bmod \left(\mathbb{A}^{1} \times \operatorname{Bun}_{G} \times \operatorname{Bun}_{G}\right)
$$

such that:

(i) $\iota_{0}(\mathcal{Q})=\mathcal{Q}_{0}$ and $\iota_{1}(\mathcal{Q})=\mathcal{Q}_{1}$, where $\iota_{t}: \operatorname{Bun}_{G} \times \operatorname{Bun}_{G} \rightarrow \mathbb{A}^{1} \times \operatorname{Bun}_{G} \times \operatorname{Bun}_{G}$ is the closed embedding corresponding to $t \in \mathbb{A}^{1}$; 
(ii) $\mathcal{Q}$ is $\mathbb{G}_{m}$-monodromic with respect to the usual action of $\mathbb{G}_{m}$ on $\mathbb{A}^{1}$; by definition, this means that $\mathcal{Q}$ belongs to the full subcategory of $\mathrm{D}-\bmod \left(\mathbb{A}^{1} \times \operatorname{Bun}_{G} \times \operatorname{Bun}_{G}\right)$ generated by the essential image of the pullback functor

$$
\operatorname{D}-\bmod \left(\left(\mathbb{A}^{1} / \mathbb{G}_{m}\right) \times \operatorname{Bun}_{G} \times \operatorname{Bun}_{G}\right) \rightarrow \mathrm{D}-\bmod \left(\mathbb{A}^{1} \times \operatorname{Bun}_{G} \times \operatorname{Bun}_{G}\right) .
$$

Assuming we have such $Q$, we take the map $Q_{1} \rightarrow Q_{0}$ of (2.8) to be the specialization map

$$
\mathrm{Sp}_{Q}: \iota_{1}^{!}(2) \rightarrow \iota_{0}^{!}(2)
$$

which is defined by virtue of the above property (ii). The definition of the specialization map is recalled in Sect. 2.2.6 below.

The remaining part of Sect.2 is devoted to constructing an object $Q$ with the above properties (i)-(ii). Informally, $Q$ should "interpolate" between $Q_{1}$ and $Q_{0}$.

Remark 2.2.5. The construction of $Q$ is geometric, and except for Sect.2.6.2 at the very end, we do not use the characteristic 0 assumption on $k$. The idea is to first construct a group-scheme $\widetilde{G}$ over $\mathbb{A}^{1}$, which "interpolates" between the groups $G$ and $\underset{M}{P} P^{-}$, and then to construct a stack over $\mathbb{A}^{1}$ which "interpolates" between $\operatorname{Bun}_{G}$ and the stack

$$
\operatorname{Bun}_{P-}^{\mu} \underset{\operatorname{Bun}_{M}^{\mu}}{\times} \operatorname{Bun}_{P}^{\mu}
$$

from diagram (2.7).

2.2.6. The specialization map. Let $y$ be a stack. Let $\iota_{0}, \iota_{1}: y \rightarrow \mathbb{A}^{1} \times y$ denote the closed embeddings corresponding to $0 \in \mathbb{A}^{1}$ and $1 \in \mathbb{A}^{1}$, respectively. Suppose that $\mathcal{K} \in \operatorname{D}-\bmod \left(\mathbb{A}^{1} \times y\right)$ is $\mathbb{G}_{m}$-monodromic with respect to the usual action of $\mathbb{G}_{m}$ on $\mathbb{A}^{1}$. In this situation we can define the specialization morphism

$$
\operatorname{Sp}_{\mathcal{K}}: \iota_{1}(\mathcal{K}) \rightarrow \iota_{0}(\mathcal{K})
$$

We need the following lemma (which is actually a particular case of Proposition 4.1.5(2)):

Lemma 2.2.7. Let $\pi: \mathbb{A}^{1} \times y \rightarrow y$ denote the projection. Then the functor $\pi_{!}$, left adjoint to $\pi^{!}$, is defined and is canonically isomorphic to $\iota_{0}^{!}$on $\mathbb{G}_{m}$-monodromic objects of $\mathrm{D}-\bmod \left(\mathbb{A}^{1} \times \mathrm{y}\right)$.

Proof. The morphism in one direction

$$
\iota_{0} \rightarrow \pi !
$$

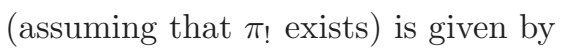

$$
\iota_{0}^{!} \simeq \pi_{!} \circ\left(\iota_{0}\right) ! \circ \iota_{0}^{!} \rightarrow \pi !
$$

To verify the existence of $\pi_{\text {! }}$ and the fact that the above map is an isomorphism, it suffices to do so for the corresponding functors $\mathrm{D}-\bmod \left(\left(\mathbb{A}^{1} / \mathbb{G}_{m}\right) \times y\right) \rightarrow \mathrm{D}-\bmod \left(\left(\mathrm{pt} / \mathbb{G}_{m}\right) \times y\right)$. Since

$$
\operatorname{D}-\bmod \left(\left(\mathbb{A}^{1} / \mathbb{G}_{m}\right) \times y\right) \simeq \operatorname{D}-\bmod \left(\mathbb{A}^{1} / \mathbb{G}_{m}\right) \otimes \operatorname{D}-\bmod (y)
$$

and

$$
\mathrm{D}-\bmod \left(\left(\mathrm{pt} / \mathbb{G}_{m}\right) \times y\right) \simeq \mathrm{D}-\bmod \left(\mathrm{pt} / \mathbb{G}_{m}\right) \otimes \mathrm{D}-\bmod (y),
$$

the assertion reduces to the case when $y=p t$, which is straightforward.

Now define the specialization morphism (2.9) to be the composition

$$
\iota_{1}^{\vdots}(\mathcal{K}) \simeq \pi_{!} \circ\left(\iota_{1}\right) ! \circ \iota_{1}(\mathcal{K}) \rightarrow \pi_{!}(\mathcal{K}) \simeq \iota_{0}^{!}(\mathcal{K})
$$

(note that since $\iota_{1}$ is a closed embedding the functor $\left(\iota_{1}\right)$ ! is well-defined). 
Remark 2.2.8. One can show (using only general nonsense) that the above composition is equal to the composition

$$
\iota_{1}^{!}(\mathcal{K}) \rightarrow \iota_{1}^{\circ} \circ \pi^{!} \circ \pi_{!}(\mathcal{K}) \simeq \pi_{!}(\mathcal{K}) \simeq \iota_{0}^{!}(\mathcal{K}) .
$$

2.3. Recollections from DrGa3. In this subsection we will recall some constructions from DrGa3, Sects. 1-2], to be subsequently applied to the group $G$.

2.3.1. Attractors and repellers. Let $Z$ be a quasi-compact scheme acted on by $\mathbb{G}_{m}$. According to [DrGa3, Proposition 1.3.4 and Corollary 1.5.3(ii)], there exist quasi-compact schemes $Z^{0}$, $Z^{+}$, and $Z^{-}$representing the following functors:

$$
\begin{gathered}
\operatorname{Maps}\left(S, Z^{0}\right)=\operatorname{Maps}^{\mathbb{G}_{m}}(S, Z), \\
\operatorname{Maps}\left(S, Z^{+}\right)=\operatorname{Maps}^{\mathbb{G}_{m}}\left(\mathbb{A}^{1} \times S, Z\right), \\
\operatorname{Maps}\left(S, Z^{-}\right)=\operatorname{Maps}^{\mathbb{G}_{m}}\left(\mathbb{A}_{-}^{1} \times S, Z\right),
\end{gathered}
$$

where $S$ is a test affine scheme and $\mathbb{A}^{1}$ (resp. $\mathbb{A}_{-}^{1}$ ) is the affine line equipped with the usual $\mathbb{G}_{m}$-action (resp. the $\mathbb{G}_{m}$-action opposite to the usual one).

The scheme $Z^{0}$ (resp. $Z^{+}$and $Z^{-}$) is called the scheme of $\mathbb{G}_{m}$-fixed points (resp. the attractor and repeller).

Let $p^{+}: Z^{+} \rightarrow Z$ and $q^{+}: Z^{+} \rightarrow Z^{0}$ denote the maps corresponding to evaluating a $\mathbb{G}_{m^{-}}$ equivariant morphism $\mathbb{A}^{1} \times S \rightarrow Z$ at $1 \in \mathbb{A}^{1}$ and $0 \in \mathbb{A}^{1}$, respectively. One defines $p^{-}: Z^{-} \rightarrow Z$ and $q^{-}: Z^{-} \rightarrow Z^{0}$ similarly.

Let $i^{+}: Z^{0} \rightarrow Z^{+}$(resp. $i^{-}: Z^{0} \rightarrow Z^{-}$) denote the morphism induced by the projection $\mathbb{A}^{1} \times S \rightarrow S$ (resp. $\mathbb{A}_{-}^{1} \times S \rightarrow S$ ).

If $Z$ is affine (the case of interest for us) then the existence of $Z^{0}$ and $Z^{ \pm}$(i.e., the representability of the corresponding functors) is very easy to prove. Moreover, in this case $Z^{0}$ and $Z^{ \pm}$are affine, and the morphisms $p^{ \pm}: Z^{ \pm} \rightarrow Z$ are closed embeddings.

2.3.2. The family of hyperbolas. We now consider the following family of curves over $\mathbb{A}^{1}$, denoted by $\mathbb{X}$ : as a scheme, $\mathbb{X}=\mathbb{A}^{2}$, and the map $\mathbb{X} \rightarrow \mathbb{A}^{1}$ is $\left(t_{1}, t_{2}\right) \mapsto t_{1} \cdot t_{2}$. The fibers of this map are hyperbolas; the zero fiber is the coordinate cross, i.e., a degenerate hyperbola.

We let $\mathbb{G}_{m}$ act on $\mathbb{X}$ hyperbolically:

$$
\lambda \cdot\left(t_{1}, t_{2}\right)=\left(\lambda \cdot t_{1}, \lambda^{-1} \cdot t_{2}\right) .
$$

2.3.3. The scheme $\widetilde{Z}$. According to DrGa3, Theorem 2.4.2], there exists a quasi-compact scheme $\widetilde{Z}$ over $\mathbb{A}^{1}$ representing the following functor on the category of schemes over $\mathbb{A}^{1}$ :

$$
\operatorname{Maps}_{\mathbb{A}^{1}}(S, \widetilde{Z}):=\operatorname{Maps}^{\mathbb{G}_{m}}\left(\underset{\mathbb{A}^{1}}{\mathbb{X}} S, Z\right) .
$$

Again, if $Z$ is affine the existence of $\widetilde{Z}$ is very easy to prove; moreover, in this case $\widetilde{Z}$ is affine. Let us also mention that if $Z$ is affine and smooth (the case of interest for us) then Proposition 2.3.8(ii) below gives a description of $\widetilde{Z}$ which some readers may prefer to consider as a definition. 
2.3.4. One has a canonical map $\widetilde{p}: \widetilde{Z} \rightarrow \mathbb{A}^{1} \times Z \times Z$. To define it, first note that any section $\sigma: \mathbb{A}^{1} \rightarrow \mathbb{X}$ of the morphism $\mathbb{X} \rightarrow \mathbb{A}^{1}$ defines a map

$$
\sigma^{*}: \operatorname{Maps}^{\mathbb{G}_{m}}\left(\mathbb{X} \times \mathbb{A}^{1} S, Z\right) \rightarrow \operatorname{Maps}(S, Z)
$$

and therefore a morphism $\widetilde{Z} \rightarrow Z$. Let $\pi_{1}: \widetilde{Z} \rightarrow Z$ and $\pi_{2}: \widetilde{Z} \rightarrow Z$ denote the morphisms corresponding to the sections

$$
t \mapsto(1, t) \in \mathbb{X} \quad \text { and } \quad t \mapsto(t, 1) \in \mathbb{X},
$$

respectively.

Finally, define

$$
\widetilde{p}: \widetilde{Z} \rightarrow \mathbb{A}^{1} \times Z \times Z
$$

to be the morphism whose first component is the tautological projection $\widetilde{Z} \rightarrow \mathbb{A}^{1}$, and the second and the third components are $\pi_{1}$ and $\pi_{2}$, respectively.

It is easy to check that if $Z$ is affine then $\widetilde{p}$ is a closed embedding (see DrGa3, Proposition 2.3.6]). So $\widetilde{p}$ identifies $\widetilde{Z}$ with a closed subscheme of $\mathbb{A}^{1} \times Z \times Z$.

2.3.5. Let $\widetilde{Z}_{t}$ denote the preimage of $t \in \mathbb{A}^{1}$ under the projection $\widetilde{Z} \rightarrow \mathbb{A}^{1}$. Let $\widetilde{p}_{t}$ denote the corresponding map $\widetilde{Z}_{t} \rightarrow Z \times Z$.

By definition, $\left(\widetilde{Z}_{1}, \widetilde{p}_{1}\right)$ identifies with $\left(Z, \Delta_{Z}\right)$. For any $t \in \mathbb{A}^{1}-\{0\}$, the pair $\left(\widetilde{Z}_{t}, \widetilde{p}_{t}\right)$ is the graph of the action of $t \in \mathbb{G}_{m}$ on $Z$. Moreover, the morphism $\tilde{p}$ induces an isomorphism

$$
\mathbb{G}_{m} \underset{\mathbb{A}^{1}}{\times} \widetilde{Z} \stackrel{\sim}{\longrightarrow} \Gamma, \quad \Gamma:=\left\{\left(t, z_{1}, z_{2}\right) \mid t \cdot z_{1}=z_{2}\right\} .
$$

The scheme $\widetilde{Z}_{0}$ identifies with $Z^{+} \underset{Z^{0}}{\times} Z^{-}$so that the morphism $\widetilde{p}_{0}: \widetilde{Z}_{0} \rightarrow Z \times Z$ identifies with the composition

$$
Z^{+} \underset{Z^{0}}{\times} Z^{-} \hookrightarrow Z^{+} \times Z^{-} \stackrel{p^{+} \times p^{-}}{\longrightarrow} Z \times Z
$$

The above-mentioned identification comes from the fact that the coordinate cross $\mathbb{X}_{0}$ is a union of $\mathbb{A}^{1}$ and $\mathbb{A}_{-}^{1}$ glued together along 0, see [DrGa3, Proposition 2.2.9].

2.3.6. The action of $\mathbb{G}_{m} \times \mathbb{G}_{m}$. In what follows we will need the action of $\mathbb{G}_{m} \times \mathbb{G}_{m}$ on $\widetilde{Z}$ that corresponds to the following action of $\mathbb{G}_{m} \times \mathbb{G}_{m}$ on $\mathbb{X}$ :

$$
\left(\lambda_{1}, \lambda_{2}\right) \cdot\left(t_{1}, t_{2}\right)=\left(\lambda_{1} \cdot t_{1}, \lambda_{2} \cdot t_{2}\right) ; \quad \lambda_{1}, \lambda_{2} \in \mathbb{G}_{m}, \quad\left(t_{1}, t_{2}\right) \in \mathbb{X}
$$

Note that the map $\widetilde{p}$ is equivariant with respect to the following action of $\mathbb{G}_{m} \times \mathbb{G}_{m}$ on $\mathbb{A}^{1} \times Z \times Z$ :

$$
\left(\lambda_{1}, \lambda_{2}\right) \cdot\left(t, z_{1}, z_{2}\right)=\left(\lambda_{1}^{-1} \cdot \lambda_{2}^{-1} \cdot t, \lambda_{1} \cdot z_{1}, \lambda_{2}^{-1} \cdot z_{2}\right) .
$$

2.3.7. Smoothness. The following facts are proved in DrGa3, Propositions 2.5.2 and 2.5.5].

Proposition 2.3.8.

(i) If $Z$ is smooth then so is the morphism $\widetilde{Z} \rightarrow \mathbb{A}^{1}$.

(ii) If $Z$ is smooth and affine then the morphism $\widetilde{p}: \widetilde{Z} \rightarrow \mathbb{A}^{1} \times Z \times Z$ induces an isomorphism

$$
\widetilde{Z} \stackrel{\sim}{\longrightarrow} \bar{\Gamma}
$$

where $\Gamma$ is as in formula (2.11) and $\bar{\Gamma}$ is the scheme-theoretic closure of $\Gamma$ in $\mathbb{A}^{1} \times Z \times Z$.

We will need the proposition only in the case that $Z$ is affine. Let us give a proof in this case, which is different from the one in DrGa3. 
Proof. We can assume that $Z$ has pure dimension $n$. This easily implies that $\widetilde{Z}_{0}=Z^{+} \underset{Z^{0}}{\times} Z^{-}$ is smooth and of pure dimension $n$.

Since $Z$ is affine, $\widetilde{p}$ is a closed embedding; so we can consider $\widetilde{Z}$ as a closed subscheme of $\mathbb{A}^{1} \times Z \times Z$. We have $\widetilde{Z} \cap\left(\mathbb{G}_{m} \times Z \times Z\right)=\Gamma$, so $\bar{\Gamma} \subset \widetilde{Z}$.

Let us show that

$$
(\bar{\Gamma})_{t}=\widetilde{Z}_{t} \text { for all } t \in \mathbb{A}^{1}
$$

(here $(\bar{\Gamma})_{t}$ is the fiber over $t$ ). The only nontrivial case is $t=0$. Both $(\bar{\Gamma})_{0}$ and $\widetilde{Z}_{0}$ have pure dimension $n$, and $\widetilde{Z}_{0}$ is smooth. Since $(\bar{\Gamma})_{0} \subset \widetilde{Z}_{0}$, it remains to check that $(\bar{\Gamma})_{0}$ meets each connected component of $\widetilde{Z}_{0}$. This follows from the obvious inclusion $\widetilde{Z} \supset \mathbb{A}^{1} \times \Delta_{Z}\left(Z^{0}\right)$.

By definition, $\bar{\Gamma}$ is flat over $\mathbb{A}^{1}$. By (2.12),$(\bar{\Gamma})_{t}$ is smooth for each $t \in \mathbb{A}^{1}$. So $\bar{\Gamma}$ is smooth over $\mathbb{A}^{1}$. It remains to show that the emebdding $\bar{\Gamma} \hookrightarrow \widetilde{Z}$ is an isomorphism. By (2.12), it suffices to check that it is an open embedding. This follows from the next lemma.

Lemma 2.3.9. Let $Y$ and $Y^{\prime}$ be schemes of finite type over a Noetherian scheme $S$, and let $f: Y^{\prime} \rightarrow Y$ be an $S$-morphism. Assume that:

(i) $Y^{\prime}$ is flat over $S$,

(ii) for any $s \in S$ the morphism $Y_{s}^{\prime} \rightarrow Y_{s}$ induced by $f$ is an open embedding.

Then $f$ is an open embedding.

This follows from Grothendieck's "Critère de platitude par fibres" (Corollary 11.3.11 from EGA IV-3). Here is a direct proof (which is much shorter than the proof of "Critère de platitude par fibres"):

Proof. It suffices to show that $f$ is étale at any $y^{\prime} \in Y^{\prime}$. Let $y$ and $s$ be the images of $y^{\prime}$ in $Y$ and $S$. Let $A$ be the completed local ring of $S$ at $s$. Let $B$ be the completed local ring of $Y$ at $y$. Let $B^{\prime}$ be the completed local ring of $Y^{\prime}$ at $y^{\prime}$. The problem is to show that the homomorphism $\varphi: B \rightarrow B^{\prime}$ induced by $f$ is an isomorphism.

By assumption, $\varphi$ induces an isomorphism $B / m B \stackrel{\sim}{\longrightarrow} B^{\prime} / m B^{\prime}$, where $m$ is the maximal ideal of $A$. Since $B^{\prime}$ is $m$-adically complete this implies that $\varphi$ is surjective. It remains to show that the ideal $I:=\operatorname{Ker}\left(B \rightarrow B^{\prime}\right)$ equals 0 . Since $B^{\prime}$ is flat over $A$ we have an exact sequence

$$
0 \rightarrow I / m I \rightarrow B / m B \rightarrow B^{\prime} / m B^{\prime} \rightarrow 0 .
$$

So $I / m I=0$. By Nakayama's lemma, this implies that $I=0$.

\subsection{The interpolating family of groups.}

2.4.1. Fix a co-character

$$
\gamma: \mathbb{G}_{m} \rightarrow M
$$

mapping to the center of $M$, which is dominant and regular with respect to $P$.

We will apply the set-up of Sect. 2.3 when $Z=G$ and the $\mathbb{G}_{m}$-action on $G$ is the adjoint action corresponding to the co-character (2.13). 
2.4.2. In this situation $Z^{+}=P, Z^{-}=P^{-}$, and $Z^{0}=M$.

Recall that the scheme

$$
\widetilde{G}:=\widetilde{Z}
$$

is a closed subscheme of $\mathbb{A}^{1} \times G \times G$.

Since the constructions of Sect. 2.3 are functorial in $Z$, this subscheme is a group-scheme over $\mathbb{A}^{1}$, which is a closed group-subscheme of the constant group-scheme $\mathbb{A}^{1} \times G \times G$. By Proposition 2.3.8, $\widetilde{G}$ is smooth over $\mathbb{A}^{1}$.

The fiber $\widetilde{G}_{1}$ of $\widetilde{G}$ over $1 \in \mathbb{A}^{1}$ is the diagonal copy of $G$, and the fiber $\widetilde{G}_{0}$ over $0 \in \mathbb{A}^{1}$ is $P \underset{M}{\times} P^{-}$.

2.4.3. Since $\widetilde{G}$ is flat over $\mathbb{A}^{1}$ the quotient $\left(\mathbb{A}^{1} \times G \times G\right) / \widetilde{G}$ exists as an algebraic space of finite type over $\mathbb{A}^{1}$. In Appendix $\mathbb{C}$ we will prove the following statement.

Proposition 2.4.4. The quotient $\left(\mathbb{A}^{1} \times G \times G\right) / \widetilde{G}$ is a quasi-affine scheme.

2.4.5. In Appendix $\mathrm{D}$ we will show how the group scheme $\widetilde{G}$ can be described using the Vinberg semi-group corresponding to $G$, see Proposition D.6.4 This description immediately implies Proposition 2.4.4 (see Corollary D.6.5). However, the proof of Proposition 2.4.4 given in Appendix $\mathrm{C}$ has the advantage of being short and self-contained.

\subsection{The interpolation of moduli of bundles.}

2.5.1. The functor that assigns to an affine scheme $S$ over $\mathbb{A}^{1}$ the groupoid of torsors on $S \times X$ with respect to the group-scheme $\left(\widetilde{G} \underset{\mathbb{A}^{1}}{ } \times S\right) \times X$ is an (a priori, non-algebraic) stack over $\mathbb{A}^{1}$, denoted by $\operatorname{Bun}_{\widetilde{G}}$. The morphism

$$
\widetilde{p}: \widetilde{G} \rightarrow \mathbb{A}^{1} \times G \times G
$$

gives rise to a morphism 9

$$
\widetilde{\mathrm{p}}: \operatorname{Bun}_{\widetilde{G}} \rightarrow \mathbb{A}^{1} \times \operatorname{Bun}_{G} \times \operatorname{Bun}_{G}
$$

\section{Proposition 2.5.2.}

(i) Bun $_{\widetilde{G}}$ is an algebraic stack smooth over $\mathbb{A}^{1}$, with an affine diagonal.

(ii) The map $\widetilde{\mathrm{p}}$ is of finite type and representable. Moreover, it is schematic.

Proof. Point (ii) follows from Proposition 2.4.4.

Let us prove (i). Statement (ii) implies that the stack Bun $\widetilde{G}$ is algebraic and locally of finite type. The fact that it has an affine diagonal is immediate from the fact that $\widetilde{G}$ itself is affine over $\mathbb{A}^{1}$. It remains to check that the morphism $\operatorname{Bun}_{\widetilde{G}} \rightarrow \mathbb{A}^{1}$ is formally smooth. As usual, this follows from the fact that any coherent sheaf on $X$ has a trivial $H^{2}$.

\footnotetext{
${ }^{9}$ We warn the reader of the clash of notations: the map $\widetilde{p}: \operatorname{Bun}_{\widetilde{G}} \rightarrow \mathbb{A}^{1} \times \operatorname{Bun}_{G} \times \operatorname{Bun}_{G}$ introduced above has nothing to do with the map $\widetilde{\mathrm{p}}: \widetilde{\operatorname{Bun}}_{P} \rightarrow \operatorname{Bun}_{G}$ of Sect. 1.1.4 The symbol $\widetilde{\mathrm{p}}$ has been chosen in both cases in order to be consistent with both $[\mathrm{DrGa3}]$ and $\left[\mathrm{BG}\right.$. The two are unlikely to be confused, as the stack $\widetilde{\operatorname{Bun}}_{P}$ will not appear again in this paper.
} 
2.5.3. Consider the action of $\mathbb{G}_{m} \times \mathbb{G}_{m}$ on $\widetilde{G}$ defined as in Sect. 2.3.6. The morphism $\widetilde{G} \rightarrow \mathbb{A}^{1}$ is $\mathbb{G}_{m} \times \mathbb{G}_{m}$-equivariant if $\mathbb{A}^{1}$ is equipped with the following $\mathbb{G}_{m} \times \mathbb{G}_{m}$-action:

$$
\left(\lambda_{1}, \lambda_{2}\right) \cdot t=\lambda_{1}^{-1} \cdot \lambda_{2}^{-1} \cdot t .
$$

Moreover, the action of $\mathbb{G}_{m} \times \mathbb{G}_{m}$ on $\widetilde{G}$ respects the group structure on $\widetilde{G}$. Therefore it induces a $\mathbb{G}_{m} \times \mathbb{G}_{m}$-action on the stack Bun $\widetilde{G}$, which covers the above $\mathbb{G}_{m} \times \mathbb{G}_{m}$-action on $\mathbb{A}^{1}$.

Lemma 2.5.4. The map $\widetilde{\mathrm{p}}: \mathrm{Bun}_{\widetilde{G}} \rightarrow \mathbb{A}^{1} \times \operatorname{Bun}_{G} \times \operatorname{Bun}_{G}$ is equivariant with respect to the above $\mathbb{G}_{m} \times \mathbb{G}_{m}$-action on $\operatorname{Bun}_{\widetilde{G}}$ and the $\mathbb{G}_{m} \times \mathbb{G}_{m}$-action on $\mathbb{A}^{1} \times \operatorname{Bun}_{G} \times \operatorname{Bun}_{G}$ via the $\mathbb{A}^{1}$-factor.

Proof. This follows from the $\mathbb{G}_{m} \times \mathbb{G}_{m}$-equivariance of the map

$$
\widetilde{p}: \widetilde{G} \rightarrow \mathbb{A}^{1} \times G \times G
$$

(see Sect. 2.3.6), and the fact that since the $\mathbb{G}_{m} \times \mathbb{G}_{m}$-action on $G \times G$ is inner, the induced action on $\operatorname{Bun}_{G} \times \operatorname{Bun}_{G}$ is canonically isomorphic to the trivial one.

Remark 2.5.5. The action on Bun $\widetilde{G}$ of the subgroup

$$
\left\{\left(\lambda_{1}, \lambda_{2}\right) \in \mathbb{G}_{m} \times \mathbb{G}_{m} \mid \lambda_{1} \cdot \lambda_{2}=1\right\} \subset \mathbb{G}_{m} \times \mathbb{G}_{m}
$$

is canonically trivial because its action on $\widetilde{G}$ is inner. Moreover, the action of the subgroup (2.15) on the triple $\left(\operatorname{Bun}_{\widetilde{G}}, \mathbb{A}^{1} \times \operatorname{Bun}_{G} \times \operatorname{Bun}_{G}, \widetilde{p}\right)$ is canonically trivial.

2.5.6. Let $\left(\operatorname{Bun}_{\widetilde{G}}\right)_{t}$ denote the fiber if $\operatorname{Bun}_{\widetilde{G}}$ over $t \in \mathbb{A}^{1}$. Let

$$
\widetilde{\mathrm{p}}_{t}:\left(\operatorname{Bun}_{\widetilde{G}}\right)_{t} \rightarrow \operatorname{Bun}_{G} \times \operatorname{Bun}_{G}
$$

denote the corresponding map.

By construction, $\left(\operatorname{Bun}_{\widetilde{G}}\right)_{1}$ identifies with $\operatorname{Bun}_{\widetilde{G}_{1}}=\operatorname{Bun}_{G}$, and the morphism

$$
\widetilde{\mathrm{p}}_{1}:\left(\operatorname{Bun}_{\widetilde{G}}\right)_{1}: \rightarrow \operatorname{Bun}_{G} \times \operatorname{Bun}_{G},
$$

identifies with the diagonal

$$
\Delta_{\mathrm{Bun}_{G}}: \operatorname{Bun}_{G} \rightarrow \operatorname{Bun}_{G} \times \operatorname{Bun}_{G} .
$$

Similarly, $\left(\operatorname{Bun}_{\widetilde{G}}\right)_{0}$ identifies with $\operatorname{Bun}_{\widetilde{G}_{0}} \simeq \operatorname{Bun}_{P \underset{M}{\times P^{-}}}$. We now claim:

Lemma 2.5.7. The natural map $\operatorname{Bun}_{P \times_{M} P^{-}} \rightarrow \operatorname{Bun}_{P} \underset{\operatorname{Bun}_{M}}{\times \operatorname{Bun}_{P^{-}}}$is an isomorphism.

The above lemma is a particular case of the following one.

Lemma 2.5.8. Let

$$
H_{1} \stackrel{f_{1}}{\longrightarrow} H \stackrel{f_{2}}{\longleftarrow} H_{2}
$$

be a diagram of algebraic groups.

(i) If $f_{1}\left(H_{1}\right) \cdot f_{2}\left(H_{2}\right)=H$ then the natural map $\operatorname{Bun}_{H_{1} \times H_{2}} \rightarrow \operatorname{Bun}_{H_{1}} \underset{\operatorname{Bun}_{H}}{\times \operatorname{Bun}_{H_{2}}}$ is an isomorphism.

(ii) If $f_{1}\left(H_{1}\right) \cdot f_{2}\left(H_{2}\right)$ is open in $H$ then the above map is an open embedding.

Proof. It suffices to show that the morphism of stacks

$$
\mathrm{pt} /\left(H_{1} \underset{H}{\times} H_{2}\right) \rightarrow \mathrm{pt} / H_{1} \underset{\mathrm{pt} / H}{\times} \mathrm{pt} / H_{2}
$$

is an isomorphism if (i) holds and an open embedding if (ii) holds. To this end, consider the action of $H_{1} \times H_{2}$ on $H$ defined by

$$
\left(h_{1}, h_{2}\right) * h:=f_{1}\left(h_{1}\right) \cdot h \cdot f_{2}\left(h_{2}\right)^{-1}, \quad h_{i} \in H_{i}, h \in H
$$


and note that the morphism (2.16) can be obtained from the embedding $f_{1}\left(H_{1}\right) \cdot f_{2}\left(H_{2}\right) \hookrightarrow H$ by passing to the quotient with respect to the action of $H_{1} \times H_{2}$.

\subsection{The interpolating kernel.}

2.6.1. Let $\operatorname{Bun}_{\widetilde{G}}^{\mu}$ denote the open substack of $\operatorname{Bun}_{\widetilde{G}}$ obtained by removing from the special fiber

$$
\operatorname{Bun}_{\widetilde{G}_{0}} \simeq \operatorname{Bun}_{P} \underset{\operatorname{Bun}_{M}}{\times} \operatorname{Bun}_{P^{-}}
$$

the union of the connected components $\operatorname{Bun}_{P}^{\mu^{\prime}} \underset{\operatorname{Bun}_{M}^{\mu^{\prime}}}{\times} \operatorname{Bun}_{P^{-}}^{\mu^{\prime}}$ with $\mu^{\prime} \neq \mu$. By a slight abuse of notation, the restriction of the map (2.14) to $\operatorname{Bun}_{\widetilde{G}}^{\mu}$ will be denoted by the same symbol

$$
\widetilde{\mathrm{p}}: \operatorname{Bun}_{\widetilde{G}}^{\mu} \rightarrow \mathbb{A}^{1} \times \operatorname{Bun}_{G} \times \operatorname{Bun}_{G} .
$$

By Lemma 2.5.7,

$$
\left(\operatorname{Bun}_{\widetilde{G}}^{\mu}\right)_{0} \simeq \operatorname{Bun}_{P}^{\mu} \underset{\operatorname{Bun}_{M}^{\mu}}{\times} \operatorname{Bun}_{P^{-}}^{\mu} .
$$

On the other hand, the map

$$
\left(\operatorname{Bun}_{\widetilde{G}}^{\mu}\right)_{1} \rightarrow\left(\operatorname{Bun}_{\widetilde{G}}\right)_{1} \simeq \operatorname{Bun}_{G}
$$

is an isomorphism.

2.6.2. By Proposition 2.5.2(ii), the morphism $\widetilde{\mathrm{p}}$ in (2.17) is quasi-compact, so the functor $\widetilde{p}_{\boldsymbol{\Delta}}$ is well-defined.

Remark 2.6.3. By Proposition 2.5.2(ii), $\widetilde{\mathrm{p}}$ is representable, and in fact schematic. As was already mentioned in Sect. 2.1.1 this implies that the morphism $\widetilde{p}_{\mathbf{\Delta}} \rightarrow \widetilde{p}_{*}$ is, in fact, an isomorphism.

$$
\begin{array}{r}
\text { We define } \mathcal{Q} \in \mathrm{D}-\bmod \left(\mathbb{A}^{1} \times \operatorname{Bun}_{G} \times \operatorname{Bun}_{G}\right) \text { by } \\
\mathcal{Q}:=\widetilde{\mathrm{p}}_{\mathbf{\Lambda}}\left(\omega_{\mathrm{Bun}_{\tilde{G}}^{\mu}}\right) .
\end{array}
$$

Let us check that $Q$ satisfies properties (i)-(ii) from Sect. 2.2.4.

2.6.4. By (2.18) and (2.19) and base change, the objects $Q_{0}$ and $Q_{1}$ from Sect. 2.2.2 identify with the !-restrictions of $Q$ under the maps

$$
\iota_{0}, \iota_{1}: \operatorname{Bun}_{G} \times \operatorname{Bun}_{G} \rightarrow \mathbb{A}^{1} \times \operatorname{Bun}_{G} \times \operatorname{Bun}_{G}
$$

corresponding to $0 \in \mathbb{A}^{1}$ and $1 \in \mathbb{A}^{1}$, respectively.

This establishes property (i).

2.6.5. By Lemma 2.5.4 and base change, $Q$ is naturally $\mathbb{G}_{m} \times \mathbb{G}_{m}$-equivariant, i.e., it is naturally a pullback of an object of

$$
\operatorname{D}-\bmod \left(\left(\mathbb{A}^{1} /\left(\mathbb{G}_{m} \times \mathbb{G}_{m}\right)\right) \times \operatorname{Bun}_{G} \times \operatorname{Bun}_{G}\right),
$$

where the $\mathbb{G}_{m} \times \mathbb{G}_{m}$-action on $\mathbb{A}^{1}$ is

$$
\left(\lambda_{1}, \lambda_{2}\right) \cdot t=\lambda_{1}^{-1} \cdot \lambda_{2}^{-1} \cdot t .
$$

In particular, $Q$ is $\mathbb{G}_{m}$-monodromic with respect to the action of $\mathbb{G}_{m}$ on $\mathbb{A}^{1}$ by dilations.

This establishes property (ii). 


\section{VERIFICATION OF ADJUNCTION}

We have the natural transformation (1.4)

$$
\mathrm{CT}_{*}^{\mu,-} \circ \operatorname{Eis}_{*}^{\mu} \rightarrow \operatorname{Id}_{\mathrm{D}-\bmod \left(\operatorname{Bun}_{M}^{\mu}\right)}
$$

and the natural transfrmation (2.1)

$$
\mathrm{Id}_{\mathrm{D}-\bmod \left(\mathrm{Bun}_{G}\right)} \rightarrow \mathrm{Eis}_{*}^{\mu} \circ \mathrm{CT}_{*}^{\mu,-},
$$

where the latter is defined using the map $Q_{1} \rightarrow Q_{0}$ of (2.8).

It remains to show that these two natural transformations satisfy the adjunction properties. That is, we have to show that the composition

$$
\mathrm{CT}_{*}^{\mu,-} \rightarrow \mathrm{CT}_{*}^{\mu,-} \circ \mathrm{Eis}_{*}^{\mu} \circ \mathrm{CT}_{*}^{\mu,-} \rightarrow \mathrm{CT}_{*}^{\mu,-}
$$

is isomorphic to the identity endomorphism of $\mathrm{CT}_{*}^{\mu,-}$, and

$$
\mathrm{Eis}_{*}^{\mu} \rightarrow \operatorname{Eis}_{*}^{\mu} \circ \mathrm{CT}_{*}^{\mu,-} \circ \mathrm{Eis}_{*}^{\mu} \rightarrow \operatorname{Eis}_{*}^{\mu}
$$

is isomorphi 10 to the identity endomorphism of $\operatorname{Eis}_{*}^{\mu}$.

We will do so for the composition (3.1). The case of (3.2) is similar and will be left to the reader.

The computation of the composition (3.1) repeats verbatim the corresponding computation in [DrGa3, Sect. 5]. We include it for the sake of completeness.

\subsection{The diagram describing the composed functor.}

3.1.1. We will use the notation

$$
\Phi:=\mathrm{CT}_{*}^{\mu,-} \circ \operatorname{Eis}_{*}^{\mu} \circ \mathrm{CT}_{*}^{\mu,-}=\left(\left(\mathrm{q}^{-}\right)_{\mathbf{\Delta}} \circ\left(\mathrm{p}^{-}\right)^{!}\right) \circ\left(\left(\mathrm{p}^{+}\right)_{\mathbf{\Delta}} \circ\left(\mathrm{q}^{+}\right)^{!}\right) \circ\left(\left(\mathrm{q}^{-}\right) \mathbf{\Delta} \circ\left(\mathrm{p}^{-}\right)^{!}\right) .
$$

By base change, $\Phi$ is given by pull-push along the following diagram:

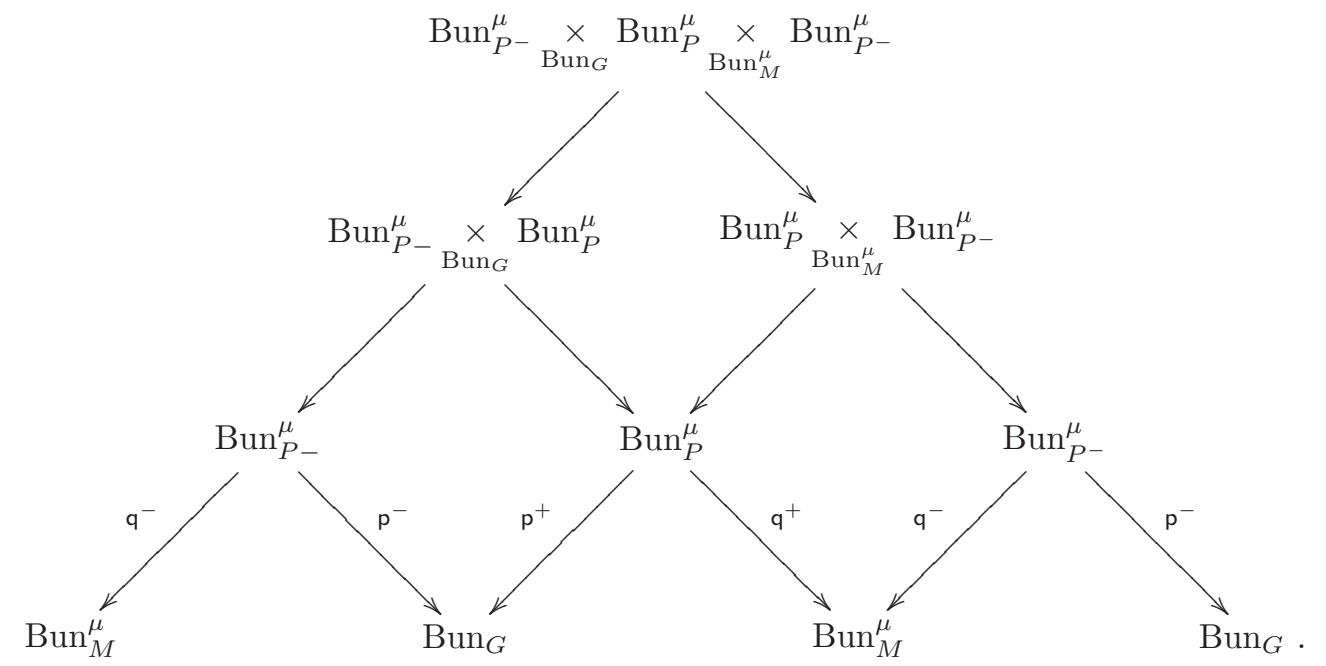

\footnotetext{
${ }^{10}$ In the future we will skip the words "isomorphic to" in similar situations. (This is a slight abuse of language since we work with the DG categories of D-modules rather than with their homotopy categories.)
} 
3.1.2. Recall the stack Bun $\underset{\widetilde{G}}{\mu}$, see Sect. 2.6.1.

Consider the stack

$$
\operatorname{Bun}_{P^{-}, \widetilde{G}}^{\mu}:=\operatorname{Bun}_{P^{-}}^{\mu} \underset{\operatorname{Bun}_{G}}{\times} \operatorname{Bun}_{\widetilde{G}}^{\mu},
$$

where the fiber product is formed using the composition

$$
\operatorname{Bun}_{\widetilde{G}}^{\mu} \stackrel{\widetilde{p}}{\longrightarrow} \mathbb{A}^{1} \times \operatorname{Bun}_{G} \times \operatorname{Bun}_{G} \rightarrow \operatorname{Bun}_{G} \times \operatorname{Bun}_{G} \stackrel{\operatorname{pr}_{1}}{\longrightarrow} \operatorname{Bun}_{G} .
$$

Let

$$
r: \operatorname{Bun}_{P^{-}, \widetilde{G}}^{\mu} \rightarrow \mathbb{A}^{1} \times \operatorname{Bun}_{M}^{\mu} \times \operatorname{Bun}_{G}
$$

denote the composition

$$
\operatorname{Bun}_{P^{-}, \widetilde{G}}^{\mu}=\operatorname{Bun}_{P^{-}}^{\mu} \underset{\operatorname{Bun}_{G}}{\times} \operatorname{Bun}_{\widetilde{G}}^{\mu} \rightarrow \mathbb{A}^{1} \times \operatorname{Bun}_{P^{-}}^{\mu} \times \operatorname{Bun}_{G} \stackrel{\operatorname{id}_{\mathbb{A}^{1}} \times \mathrm{q}^{-} \times \operatorname{id}_{\mathrm{Bun}_{G}}}{\longrightarrow} \mathbb{A}^{1} \times \operatorname{Bun}_{M}^{\mu} \times \operatorname{Bun}_{G},
$$

where the first arrow is obtained by base change from

$$
\widetilde{\mathrm{p}}: \operatorname{Bun}_{\widetilde{G}}^{\mu} \rightarrow \mathbb{A}^{1} \times \operatorname{Bun}_{G} \times \operatorname{Bun}_{G}
$$

Let $\left(\operatorname{Bun}_{P^{-}, \widetilde{G}}^{\mu}\right)_{t}$ denote the fiber of $\operatorname{Bun}_{P^{-}, \widetilde{G}}^{\mu}$ over $t \in \mathbb{A}^{1}$. Let $r_{t}$ denote the corresponding $\operatorname{map}\left(\operatorname{Bun}_{P^{-}, \widetilde{G}}^{\mu}\right)_{t} \rightarrow \operatorname{Bun}_{M}^{\mu} \times \operatorname{Bun}_{G}$.

3.1.3. The isomorphism (2.18) defines an isomorphism

$$
\left(\operatorname{Bun}_{P^{-}, \widetilde{G}^{\prime}}^{\mu}\right)_{0} \simeq \operatorname{Bun}_{P^{-}}^{\mu} \underset{\operatorname{Bun}_{G}}{\times} \operatorname{Bun}_{P}^{\mu} \underset{\operatorname{Bun}_{M}^{\mu}}{\times} \operatorname{Bun}_{P^{-}}^{\mu}
$$

such that the compositions

$$
\operatorname{Bun}_{P^{-}}^{\mu} \underset{\operatorname{Bun}_{G}}{\times} \operatorname{Bun}_{P}^{\mu} \underset{\operatorname{Bun}_{M}^{\mu}}{\times} \operatorname{Bun}_{P^{-}}^{\mu} \rightarrow \operatorname{Bun}_{P^{-}}^{\mu} \underset{\operatorname{Bun}_{G}}{\times} \operatorname{Bun}_{P}^{\mu} \rightarrow \operatorname{Bun}_{P^{-}}^{\mu} \rightarrow \operatorname{Bun}_{M}^{\mu}
$$

and

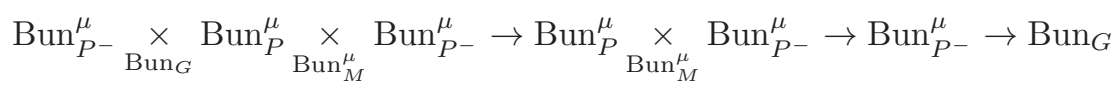

from diagram (3.4) are equal, respectively, to the compositions

$$
\left(\operatorname{Bun}_{P^{-}, \widetilde{G}}^{\mu}\right)_{0} \stackrel{\mathrm{r}}{\longrightarrow} \operatorname{Bun}_{M}^{\mu} \times \operatorname{Bun}_{G} \stackrel{\mathrm{pr}_{1}}{\longrightarrow} \operatorname{Bun}_{M}^{\mu}
$$

and

$$
\left(\operatorname{Bun}_{P^{-}, \widetilde{G}}^{\mu}\right)_{0} \stackrel{r}{\longrightarrow} \operatorname{Bun}_{M}^{\mu} \times \operatorname{Bun}_{G} \stackrel{\mathrm{pr}_{2}}{\longrightarrow} \operatorname{Bun}_{G} .
$$

Hence, the functor $\Phi$ is given by pull-push along the diagram

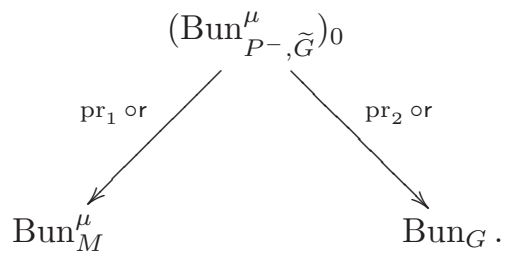

\subsection{The natural transformations at the level of kernels.}


3.2.1. Denote

$$
\mathcal{S}:=\mathbf{r}_{\mathbf{\Delta}}\left(\omega_{\mathrm{Bun}_{P^{-}, \tilde{G}}^{\mu}}\right) \in \mathrm{D}-\bmod \left(\mathbb{A}^{1} \times \operatorname{Bun}_{M}^{\mu} \times \operatorname{Bun}_{G}\right) .
$$

As in Sect. 2.6.5 one shows that

$$
\mathcal{S} \in \mathrm{D}-\bmod \left(\mathbb{A}^{1} \times \operatorname{Bun}_{M}^{\mu} \times \operatorname{Bun}_{G}\right)^{\mathbb{G}_{m}-\text { mon }} .
$$

Set also

and

$$
\mathcal{S}_{0}:=\left(r_{0}\right)_{\mathbf{\Lambda}}\left(\omega_{\left(\operatorname{Bun}_{P-, \widetilde{G}}^{\mu}\right)_{0}}\right) \in \mathrm{D}-\bmod \left(\operatorname{Bun}_{M}^{\mu} \times \operatorname{Bun}_{G}\right)
$$

$$
\mathcal{S}_{1}:=\left(\mathrm{r}_{1}\right)_{\mathbf{\Lambda}}\left(\omega_{\left(\operatorname{Bun}_{P^{-}, \widetilde{G}^{\prime}}^{\mu}\right)_{1}}\right) \in \mathrm{D}-\bmod \left(\operatorname{Bun}_{M}^{\mu} \times \operatorname{Bun}_{G}\right) .
$$

3.2.2. By Sects. 2.1.5 and 3.1.3 the functor $\Phi$ identifies with $F_{S_{0}}$. Let us now describe the natural transformations

$$
\Phi \rightarrow\left(\mathrm{p}^{-}\right) \mathbf{\Delta} \circ\left(\mathrm{q}^{-}\right)^{!} \text {and }\left(\mathrm{p}^{-}\right) \mathbf{\Delta} \circ\left(\mathrm{q}^{-}\right)^{!} \rightarrow \Phi
$$

at the level of kernels.

Set

We have

$$
\mathcal{T}:=\left(\mathrm{q}^{-} \times \mathrm{p}^{-}\right) \mathbf{\Lambda}\left(\omega_{\mathrm{Bun}_{P^{-}}^{\mu}}\right)
$$

$$
\left(\mathrm{p}^{-}\right)_{\mathbf{\Delta}} \circ\left(\mathrm{q}^{-}\right)^{!} \simeq \mathrm{F}_{\mathcal{T}}
$$

3.2.3. Recall the open embedding

$$
\mathrm{j}: \operatorname{Bun}_{M}^{\mu} \hookrightarrow \operatorname{Bun}_{P^{-}}^{\mu} \underset{\operatorname{Bun}_{G}}{\times} \operatorname{Bun}_{P}^{\mu} .
$$

Let $\mathrm{j}_{P^{-}}$denote the open embedding

$$
\operatorname{Bun}_{P^{-}}^{\mu} \hookrightarrow \operatorname{Bun}_{P^{-}}^{\mu} \underset{\operatorname{Bun}_{G}}{\times} \operatorname{Bun}_{P}^{\mu} \underset{\operatorname{Bun}_{M}^{\mu}}{\times} \operatorname{Bun}_{P^{-}}^{\mu} \simeq\left(\operatorname{Bun}_{P^{-}, \widetilde{G}^{\prime}}^{\mu}\right)_{0},
$$

obatined by base change.

We have

$$
\left(\mathrm{q}^{-} \times \mathrm{p}^{-}\right)=\mathrm{r}_{0} \circ \mathrm{j}_{P^{-}}, \quad \operatorname{Bun}_{P^{-}}^{\mu} \rightarrow \operatorname{Bun}_{M}^{\mu} \times \operatorname{Bun}_{G} .
$$

3.2.4. By construction, the natural transformation $\Phi \rightarrow\left(\mathrm{p}^{-}\right) \mathbf{\Delta} \circ\left(\mathrm{q}^{-}\right)^{!}$comes from the map

$$
\mathcal{S}_{0} \rightarrow \mathcal{T}
$$

equal to

$$
\left(r_{0}\right)_{\mathbf{\Delta}}\left(\omega_{\left(\operatorname{Bun}_{P^{-}, \widetilde{G}^{\prime}}^{\mu}\right)_{0}}\right) \rightarrow\left(r_{0}\right)_{\mathbf{\Delta}} \circ\left(j_{P^{-}}\right) \mathbf{\Delta}\left(\omega_{\mathrm{Bun}_{P^{-}}^{\mu}}\right) \simeq\left(\mathrm{q}^{-} \times \mathrm{p}^{-}\right) \mathbf{\Delta}\left(\omega_{\mathrm{Bun}_{P^{-}}^{\mu}}\right),
$$

where the first arrow comes from

$$
\omega_{\left(\operatorname{Bun}_{P^{-}, \widetilde{G}^{\prime}}^{\mu}\right)_{0}} \rightarrow\left(\mathrm{j}_{P^{-}}\right)_{*} \circ\left(\mathrm{j}_{P^{-}}\right)^{*}\left(\omega_{\left(\operatorname{Bun}_{P^{-}, \widetilde{G}}^{\mu}\right)_{0}}\right) \simeq\left(\mathrm{j}_{P^{-}}\right)_{*}\left(\omega_{\mathrm{Bun}_{P^{-}}^{\mu}}\right) \simeq\left(\mathrm{j}_{P^{-}}\right)_{\mathbf{\Delta}}\left(\omega_{\mathrm{Bun}_{P^{-}}^{\mu}}\right) .
$$

3.2.5. The identification $\left(\operatorname{Bun}_{\widetilde{G}}^{\mu}\right)_{1} \simeq \operatorname{Bun}_{G}$ of 2.19 gives rise to an identification

$$
\left(\operatorname{Bun}_{P^{-}, \widetilde{G}}^{\mu}\right)_{1} \simeq \operatorname{Bun}_{P^{-}}^{\mu},
$$

so that

$$
r_{1}=\left(q^{-} \times p^{-}\right)
$$

Hence, we obtain a tautological identification

$$
\mathcal{T} \simeq \mathcal{S}_{1} .
$$


3.2.6. Now, the map $\mathrm{Sp}_{\mathcal{S}}$ of (2.9) defines a canonical map

$$
\mathcal{S}_{1} \rightarrow \mathcal{S}_{0}
$$

By the functoriality of the construction of the natural transformation Sp (see, e.g., DrGa3. Sect. 4.1.5]), the natural transformation $\left(\mathrm{p}^{-}\right) \mathbf{\Delta} \circ\left(\mathrm{q}^{-}\right)^{!} \rightarrow \Phi$ comes from the map

$$
\mathcal{T} \rightarrow \mathcal{S}_{1} \rightarrow \mathcal{S}_{0},
$$

equal to the composition of (3.9) and (3.10).

3.2.7. We obtain that in order to prove that the composition (3.1) is the identity map, it suffices to show that the composed map

$$
\mathcal{T} \rightarrow \mathcal{S}_{1} \rightarrow \mathcal{S}_{0} \rightarrow \mathcal{T}
$$

is the identity map on $\mathcal{T}$.

\subsection{Passing to an open substack.}

3.3.1. Recall the open embedding

$$
\mathrm{j}_{P^{-}}: \operatorname{Bun}_{P^{-}}^{\mu} \hookrightarrow\left(\operatorname{Bun}_{P^{-}, \widetilde{G}}^{\mu}\right)_{0},
$$

introduced in Sect. 3.2 .3

Let

$$
\operatorname{Bun}_{P^{-,}, \widetilde{G}}^{\mu} \subset \operatorname{Bun}_{P^{-}, \widetilde{G}}^{\mu}
$$

be the open substack obtained by removing the closed substack

$$
\left(\left(\operatorname{Bun}_{P^{-}, \widetilde{G}}^{\mu}\right)_{0}-\mathrm{j}_{P^{-}}\left(\operatorname{Bun}_{P^{-}}^{\mu}\right)\right) \subset\left(\operatorname{Bun}_{P^{-}, \widetilde{G}}^{\mu}\right)_{0} \subset \operatorname{Bun}_{P^{-}, \widetilde{G}}^{\mu} .
$$

Let $\left({\stackrel{\circ}{\operatorname{Bun}^{-}}}_{P^{-}, \widetilde{G}}^{\mu}\right)_{t}$ denote the fiber of $\stackrel{\circ}{\mathrm{Bun}}_{P^{-}, \widetilde{G}}^{\mu}$ over $t \in \mathbb{A}^{1}$.

Note that the isomorphism $\operatorname{Bun}_{P^{-}}^{\mu} \rightarrow\left(\operatorname{Bun}_{P^{-}, \widetilde{G}}^{\mu}\right)_{1}$ of (3.8) defines an isomorphism

$$
\operatorname{Bun}_{P^{-}}^{\mu} \rightarrow\left(\stackrel{\circ}{B u n}_{P^{-}, \widetilde{G}}^{\mu}\right)_{1} .
$$

By definition, the open embedding $\mathrm{j}_{P^{-}}: \operatorname{Bun}_{P^{-}}^{\mu} \hookrightarrow\left(\operatorname{Bun}_{P^{-}, \widetilde{G}^{\prime}}^{\mu}\right)_{0}$ defines an isomorphism

$$
\operatorname{Bun}_{P^{-}}^{\mu} \rightarrow\left(\stackrel{\circ}{\operatorname{Bun}_{P^{-}, \widetilde{G}}^{\mu}}\right)_{0} .
$$

3.3.2. Let

$$
\stackrel{\circ}{\mathrm{r}}:{\stackrel{\circ}{\operatorname{Bun}^{-,}, \widetilde{G}}}_{P^{-}}^{\mu} \mathbb{A}^{1} \times \operatorname{Bun}_{M}^{\mu} \times \operatorname{Bun}_{G} \text { and } \stackrel{\circ}{\mathrm{r}}{ }_{t}:{\stackrel{\circ}{\operatorname{Bun}^{-}, \widetilde{G}}}_{P^{-}}^{\mu} \rightarrow \operatorname{Bun}_{M}^{\mu} \times \operatorname{Bun}_{G}
$$

denote the corresponding maps.

Set

and also

$$
\stackrel{\circ}{\mathcal{S}}:=\stackrel{\circ}{\mathrm{\Lambda}}\left(\underset{\operatorname{Bun}_{P^{-}, \widetilde{G}}^{\mu}}{\omega}\right),
$$

$$
\stackrel{\circ}{\mathcal{S}}_{0}:=\left(\stackrel{\circ}{\mathrm{r}}_{0}\right)_{\mathbf{\Delta}}\left(\omega_{\left(\operatorname{Bun}_{P-, \widetilde{G}}^{\mu}\right)_{0}}^{\circ}\right) \text { and } \stackrel{\circ}{\mathcal{S}}_{1}:=\left(\stackrel{\circ}{\mathrm{r}}_{1}\right)_{\mathbf{\Delta}}\left(\omega_{\left(\operatorname{Bun}_{P-, \widetilde{G}}^{\mu}\right)_{1}}^{\circ}\right) .
$$


The open embedding $\operatorname{Bun}_{P^{-}, \widetilde{G}}^{\mu} \hookrightarrow \operatorname{Bun}_{P^{-}, \widetilde{G}}^{\mu}$ gives rise to the maps

$$
\mathcal{S} \rightarrow \stackrel{\circ}{\mathcal{S}}, \quad \mathcal{S}_{0} \rightarrow \stackrel{\circ}{\mathcal{S}}_{0}, \quad \stackrel{\circ}{\mathcal{S}}_{1} \rightarrow \stackrel{\circ}{\mathcal{S}}_{1}
$$

As in Sect. 3.2, we have the natural transformations

$$
\mathcal{T} \rightarrow \stackrel{\circ}{\mathcal{S}}_{1} \rightarrow \stackrel{\circ}{\mathcal{S}}_{0} \rightarrow \mathcal{T}
$$

Moreover, the diagram

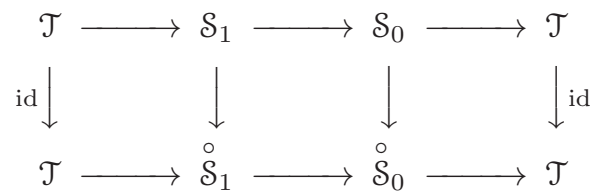

commutes.

Hence, we obtain that in order to show that the composed map (3.12) is the identity map, it suffices to show that the composed map [3.15) is the identity map.

\subsection{Digression: description of fiber products.}

3.4.1. Let $Z$ be a quasi-compact scheme equipped with a $\mathbb{G}_{m}$-action. Consider the fiber product $Z_{Z}^{-} \underset{Z}{Z}$, formed using the composition

$$
\widetilde{\widetilde{p}} \rightarrow \mathbb{A}^{1} \times Z \times Z \rightarrow Z \times Z \stackrel{\mathrm{pr}_{1}}{\rightarrow} Z
$$

In [DrGa3, Sect. 2.6] we define a canonical morphism

$$
\mathbb{A}^{1} \times Z^{-} \rightarrow Z^{-} \times \underset{Z}{Z}
$$

of schemes over $\mathbb{A}^{1}$.

3.4.2. If $Z$ is affine (the case of interest for us) the definition from DrGa3] can be reformulated as follows. Recall that if $Z$ is affine then $Z^{-}$is a closed subscheme of $Z$ and $\widetilde{Z}$ is a closed subscheme of $\mathbb{A}^{1} \times Z \times Z$, so $Z^{-} \underset{Z}{\times} \widetilde{Z}$ is a closed subscheme of $\mathbb{A}^{1} \times Z \times Z$.

Now define the map

$$
\mathbb{A}^{1} \times Z^{-} \rightarrow Z^{-} \underset{Z}{\times} \widetilde{Z} \subset \mathbb{A}^{1} \times Z \times Z
$$

by

$$
(t, z) \mapsto\left(t, t^{-1} \cdot z, z\right) \in \mathbb{A}^{1} \times Z \times Z
$$

It is easy to see that the image of the map (3.17) indeed belongs to $Z^{-} \underset{Z}{\times} \widetilde{Z}$. Indeed, it suffices to consider the case $t \neq 0$, when this is obvious.

3.4.3. By [DrGa3, Proposition 2.6.3 and Remark 2.6.4], for any $Z$ the moprhism (3.16) is an open embedding, and if $Z$ is affine it is an isomorphism.

The latter is very easy to check if $Z$ is affine and smooth: in this case the map (3.16) is a morphism between smooth schemes over $\mathbb{A}^{1}$, and it suffices to check that it induces an isomorphism between the fibers over any $k$-point of $\mathbb{A}^{1}$. 
3.4.4. Consider now the situation of Sect. 2.4.1, i.e., $Z=G$ and the $\mathbb{G}_{m}$-action on $G$ is the adjoint action corresponding to a co-character $\gamma: \mathbb{G}_{m} \rightarrow M$ such that $\gamma\left(\mathbb{G}_{m}\right)$ is contained in the center of $M$ and $\gamma$ is dominant and regular with respect to $P$.

Then the map (3.16) is a homomorphism

$$
\mathbb{A}^{1} \times P^{-} \rightarrow P^{-} \underset{G}{\times} \widetilde{G}
$$

of group-schemes over $\mathbb{A}^{1}$. Its composition with the embedding $P^{-} \underset{G}{\times} \widetilde{G} \hookrightarrow \mathbb{A}^{1} \times G \times G$ is given by

$$
(t, g) \mapsto\left(t, \gamma(t)^{-1} \cdot g \cdot \gamma(t), g\right) .
$$

3.4.5. The homorphism (3.18) induces a maps of moduli of bundles

$$
\mathbb{A}^{1} \times \operatorname{Bun}_{P^{-}} \rightarrow \operatorname{Bun}_{P^{-}} \underset{\operatorname{Bun}_{G}}{\times} \operatorname{Bun}_{\widetilde{G}} .
$$

Lemma 3.4.6. The map (3.19) is an open embedding.

This lemma is a counterpart of DrGa3, Proposition 2.6.3].

Proof. Note that Lemma 2.3 .9 remains valid for stacks. So it suffices to show that the map (3.19) induces an open embedding of fibers over any $t \in \mathbb{A}^{1}$. This is clear if $t \neq 0$, so it remains to consider the case of $t=0$.

The morphsim in question is the map

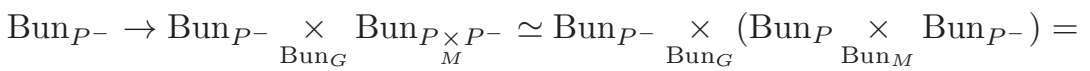

$$
\begin{aligned}
& =\left(\operatorname{Bun}_{P^{-}} \underset{\operatorname{Bun}_{G}}{\times} \operatorname{Bun}_{P}\right) \underset{\operatorname{Bun}_{M}}{\times} \operatorname{Bun}_{P^{-}}
\end{aligned}
$$

which equals the map

$$
\operatorname{Bun}_{P^{-}} \simeq \operatorname{Bun}_{M} \underset{\operatorname{Bun}_{M}}{\times} \operatorname{Bun}_{P^{-}} \stackrel{\text { jxid }}{\longrightarrow}\left(\operatorname{Bun}_{P^{-}} \underset{\operatorname{Bun}_{G}}{\times} \operatorname{Bun}_{P}\right) \underset{\operatorname{Bun}_{M}}{\times} \operatorname{Bun}_{P^{-}} .
$$

It is an open embedding because so is the morphism $\mathrm{j}: \operatorname{Bun}_{M} \rightarrow \operatorname{Bun}_{P^{-}} \underset{\operatorname{Bun}_{G}}{\times} \operatorname{Bun}_{P}$.

Remark 3.4.7. One can also prove Lemma 3.4.6 using a variant of Lemma 2.5.8(ii). In this variant $H_{1}, H_{2}$, and $H$ are flat group-schemes over some scheme $S$ (e.g., $S=\mathbb{A}^{1}$ ) and instead of openness of $f_{1}\left(H_{1}\right) \cdot f_{2}\left(H_{2}\right)$ one requires flatness of the morphism $H_{1} \underset{S}{\times} H_{2} \rightarrow H$ given by $\left(h_{1}, h_{2}\right) \mapsto f_{1}\left(h_{1}\right) \cdot f_{2}\left(h_{2}\right)$.

\subsection{The key argument.}

3.5.1. By construction, the open embedding (3.19) defines an isomorphism

$$
\mathbb{A}^{1} \times \operatorname{Bun}_{P^{-}}^{\mu} \rightarrow{\stackrel{\circ}{\operatorname{Bun}^{-}}}_{P^{-}, \widetilde{G}}^{\mu}
$$

with the following properties:

- The map

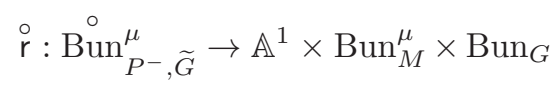

identifies with the map

$$
\operatorname{id}_{\mathbb{A}^{1}} \times\left(q^{-} \times p^{-}\right) .
$$


- The isomorphism $\operatorname{Bun}_{P^{-}}^{\mu} \rightarrow\left({\stackrel{\circ}{\operatorname{Bun}_{P^{-}, \widetilde{G}}^{\mu}}}_{)_{1}}\right.$ of (13.13) corresponds to the identity map

$$
\operatorname{Bun}_{P^{-}}^{\mu} \simeq\left(\mathbb{A}^{1} \times \operatorname{Bun}_{P^{-}}^{\mu}\right) \underset{\mathbb{A}^{1}}{\times}\{1\} \simeq \operatorname{Bun}_{P^{-}}^{\mu} .
$$

- The isomorphism $\operatorname{Bun}_{P^{-}}^{\mu} \rightarrow\left(\stackrel{\circ}{\operatorname{Bun}_{P^{-}, \widetilde{G}}^{\mu}}\right)_{0}$ of (3.14) corresponds to the identity map

$$
\operatorname{Bun}_{P^{-}}^{\mu} \simeq\left(\mathbb{A}^{1} \times \operatorname{Bun}_{P^{-}}^{\mu}\right) \underset{\mathbb{A}^{1}}{\times}\{0\} \simeq \operatorname{Bun}_{P^{-}}^{\mu} .
$$

3.5.2. Hence, we obtain that the composition (3.15) identifies with

$$
\mathcal{T} \simeq \iota_{1}^{\prime}\left(\omega_{\mathbb{A}^{1}} \otimes \mathcal{T}\right) \rightarrow \iota_{0}^{!}\left(\omega_{\mathbb{A}^{1}} \otimes \mathcal{T}\right) \simeq \mathcal{T},
$$

where

$$
\iota_{1}^{!}\left(\omega_{\mathbb{A}^{1}} \otimes \mathcal{T}\right) \rightarrow \iota_{0}^{!}\left(\omega_{\mathbb{A}^{1}} \otimes \mathcal{T}\right)
$$

is the specialization map (2.9) for the object

$$
\omega_{\mathbb{A}^{1}} \otimes \mathcal{T} \in \mathrm{D}-\bmod \left(\mathbb{A}^{1} \times z^{0} \times z\right) .
$$

However, the latter is readily seen to be the identity map.

\section{An alternative PRoOF}

In this section we will give an alternative proof of Theorem 1.2.5 by directly deducing it from Braden's theorem $([\mathrm{Br}]$ ). It has the advantage of being more elementary than the proof of Theorem 1.2.5 given in Sect. 2, if one accepts Braden's theorem as a black box.

However, the two proofs are closely related, because Braden's theorem itself can be proved by an argument similar to one used in the proof of Theorem 1.2 .5 from Sect. 2.

\subsection{Contraction principle.}

4.1.1. Let $y$ be a stack equipped with an action of $\mathbb{G}_{m}$. We let

$$
\mathrm{D}-\bmod (y)^{\mathbb{G}_{m}, \operatorname{mon}} \subset \mathrm{D}-\bmod (y)
$$

denote the corresponding monodromic subcategory, i.e., the full subcategory generated by the essential image of the pullback functor $D-\bmod \left(y / \mathbb{G}_{m}\right) \rightarrow D-\bmod (y)$.

Note that if the $\mathbb{G}_{m}$-action is trivial then $\mathrm{D}-\bmod (y)^{\mathbb{G}_{m}, \operatorname{mon}}=\mathrm{D}-\bmod (y)$ (because the morphism $y \rightarrow y / \mathbb{G}_{m}$ admits a section).

4.1.2. Let $y$ be an algebraic stack equipped with an action of $\mathbb{A}^{1}$, where $\mathbb{A}^{1}$ is viewed as a monoid with respect to multiplication. (A concrete example of this situation will be considered in Sect. 4.1.6 below).

Define the stack $y^{0}$ by

$$
\operatorname{Maps}\left(S, y^{0}\right):=\operatorname{Maps}^{\mathbb{A}^{1}}(S, y), \quad S \in \operatorname{Sch}^{\text {aff }} .
$$

The groupoid Maps $\left(S, y^{0}\right)$ admits also the following description (which was used in DrGa2, Sect. C.5] as a definition): objects of $\operatorname{Maps}\left(S, y^{0}\right)$ are pairs $(y, \alpha)$, where $y \in \operatorname{Maps}(S, y)$ and $\alpha$ is an isomorphism $0 \cdot y \stackrel{\sim}{\longrightarrow} y$ such that the two isomorphisms

$$
0 \cdot(0 \cdot y)=(0 \cdot 0) \cdot y=0 \cdot y \stackrel{\alpha}{\longrightarrow} y \text { and } 0 \cdot(0 \cdot y) \stackrel{0 \cdot \alpha}{\longrightarrow} 0 \cdot y \stackrel{\alpha}{\longrightarrow} y
$$

$0 \cdot(0 \cdot y) \rightrightarrows y$ are equal to each other. Morphisms in $\operatorname{Maps}\left(S, y^{0}\right)$ are defined as morphisms between the $y$ 's that intertwine the data of $\alpha$. 
From the latter description, it is easy to see that $y^{0}$ is again a (locally QCA) stack.

4.1.3. The forgetful map and the action of $0 \in \mathbb{A}^{1}$ define a retraction

$$
y^{0} \stackrel{\imath}{\longrightarrow} y \stackrel{q}{\longrightarrow} y^{0} .
$$

Under these circumstances we will say that the we are given a contraction of $y$ onto $y^{0} \stackrel{\imath}{\longrightarrow} y$.

Since $\mathrm{q} \circ \imath=\mathrm{id}_{\mathrm{yo}}$, it follows that $\imath$ is quasi-compact and representable.

4.1.4. From now on we will assume that the morphism $q$ is quasi-compact. In this case the functor

is defined.

$$
\mathrm{q}_{\mathbf{\Delta}}: \mathrm{D}-\bmod (\mathrm{y}) \rightarrow \mathrm{D}-\bmod \left(\mathrm{y}^{0}\right)
$$

We have the following assertion:

Proposition 4.1.5.

(1) The partially defined left adjoint $\imath^{*}: \mathrm{D}-\bmod (\mathrm{y}) \rightarrow \mathrm{D}-\bmod \left(y^{0}\right)$ of $\imath_{*} \simeq \imath_{\mathbf{\Delta}}$ is defined on $\mathrm{D}-\bmod (\mathrm{y})^{\mathbb{G}_{m}}$, mon , and we have a canonical isomorhism

$$
\left.\left.i^{*}\right|_{\mathrm{D}-\bmod (y)^{\mathbb{G}}, \operatorname{mon}} \simeq q_{\mathbf{\Delta}}\right|_{\mathrm{D}-\bmod (y)^{\mathbb{G}_{m}, \operatorname{mon}}} .
$$

More precisely, for each $\mathcal{F} \in \mathrm{D}-\bmod (y)^{\mathbb{G}_{m}}$, mon the natural map

$$
q_{\mathbf{\Delta}}(\mathcal{F}) \rightarrow q_{\mathbf{\Delta}} \circ i_{*} \circ i^{*}(\mathcal{F}) \simeq q_{\mathbf{\Delta}} \circ i_{\mathbf{\Delta}} \circ i^{*}(\mathcal{F})=(q \circ i)_{\mathbf{\Delta}} \circ i^{*}(\mathcal{F})=i^{*}(\mathcal{F})
$$

is an isomorphism.

(2) The partially defined left adjoint $q !: \mathrm{D}-\bmod (\mathrm{y}) \rightarrow \mathrm{D}-\bmod \left(\mathrm{y}^{0}\right)$ of $q^{!}$is defined on $\mathrm{D}-\bmod (\mathrm{y})^{\mathbb{G}_{m}}$, mon , and we have a canonical isomorhism

$$
\left.\left.q !\right|_{\mathrm{D}-\bmod (y)^{\mathbb{G}_{m}, \operatorname{mon}}} \simeq i^{!}\right|_{\mathrm{D}-\bmod (y)^{\mathbb{G}_{m}, \operatorname{mon}}} .
$$

More precisely, for each $\mathcal{F} \in \mathrm{D}-\bmod (y)^{\mathbb{G}_{m}}$, mon the natural map

$$
i^{!}(\mathcal{F}) \rightarrow i^{!} \circ q^{!} \circ q_{!}(\mathcal{F})=(q \circ i)^{!} \circ q_{!}(\mathcal{F})=q_{!}(\mathcal{F})
$$

is an isomorphism.

For the proof see [DrGa2, Theorem C.5.3].

\subsubsection{A contraction of $\operatorname{Bun}_{P}$ onto $\operatorname{Bun}_{M}$.}

Let us take $y=\operatorname{Bun}_{P}$. Let $\gamma: \mathbb{G}_{m} \rightarrow M$ be a co-character as in (2.13). The resulting adjoint action of $\mathbb{G}_{m}$ on $P$ extends to an action of $\mathbb{A}^{1}$ on $P$ such that $0 \in \mathbb{A}^{1}$ acts as

$$
P \rightarrow M=P \cap P^{-} \hookrightarrow P .
$$

The action of $\mathbb{A}^{1}$ on $P$ induces on action of $\mathbb{A}^{1}$ on $\operatorname{Bun}_{P}$.

It is easy to check that the resulting diagram (4.1) identifies with

$$
\operatorname{Bun}_{M} \stackrel{\iota}{\longrightarrow} \operatorname{Bun}_{P} \stackrel{\mathrm{q}}{\longrightarrow} \operatorname{Bun}_{M},
$$

where the maps $\iota$ and q come from the above homomorphisms $M \hookrightarrow P$ and $P \rightarrow M$.

Note that the $\mathbb{G}_{m}$-action on $\operatorname{Bun}_{P}$, corresponding to the above $\mathbb{A}^{1}$-action, is canonically isomorphic to the trivial on 11 (because the $\mathbb{G}_{m}$-action on $P$ comes from a homomorphism $\mathbb{G}_{m} \rightarrow P$ and the conjugation action of $P$ on itself).

\footnotetext{
${ }^{11}$ This trivialization is not compatible with the projection $\mathrm{q}: \mathrm{Bun}_{P} \rightarrow \operatorname{Bun}_{M}$, so the $\mathbb{G}_{m}$-action on the fibers of the morphism $\operatorname{Bun}_{P} \rightarrow \operatorname{Bun}_{M}$ is non-trivial!
} 
Therefore, the inclusion

$$
\mathrm{D}-\bmod \left(\operatorname{Bun}_{P}\right)^{\mathbb{G}_{m}, \operatorname{mon}} \subset \mathrm{D}-\bmod \left(\operatorname{Bun}_{P}\right)
$$

is an equality. So by Proposition 4.1.5, the functors

$$
\mathrm{q}_{!}: \mathrm{D}-\bmod \left(\operatorname{Bun}_{P}\right) \rightarrow \mathrm{D}-\bmod \left(\operatorname{Bun}_{M}\right) \text { and } \iota^{*}: \mathrm{D}-\bmod \left(\operatorname{Bun}_{P}\right) \rightarrow \mathrm{D}-\bmod \left(\operatorname{Bun}_{M}\right)
$$

left adjoint to $\mathrm{q}^{!}$and $\iota_{*} \simeq \iota_{\mathbf{\Delta}}$ are defined and are canonically isomorhic to $\iota^{!}$and $\mathrm{q}_{\mathbf{\Delta}}$, respectively.

Note also that in this example, $\mathrm{q}_{\mathbf{\Delta}} \simeq \mathrm{q}_{*}$, because the morphism $\mathrm{q}$ is safe.

The same is, of course, true for $P$ replaced by $P^{-}$, when we replace the above action of $\mathbb{G}_{m}$ by its inverse.

4.2. Hyperbolic restrictions and Braden's theorem. In this subsection we will recall the statement of Braden's theorem in the set-up and notations of [DrGa3, Sect. 3.1].

4.2.1. The material in this subsection uses the notion of pro-completion $\operatorname{Pro}(\mathbf{C})$ of a DG category $\mathbf{C}$; we refer the reader to $[\mathrm{DrGa3}$, Appendix A], where the corresponding definitions are given.

Let $f: y_{1} \rightarrow y_{2}$ be a quasi-compact and safe morphism between stacks. Consider the functors

$$
f^{!}: \mathrm{D}-\bmod \left(y_{2}\right) \rightarrow \mathrm{D}-\bmod \left(y_{1}\right) \text { and } f_{*} \simeq f_{\mathbf{\Delta}}: \mathrm{D}-\bmod \left(y_{1}\right) \rightarrow \mathrm{D}-\bmod \left(y_{2}\right),
$$

We will regard their partially defined left adjoints as functors

$$
f_{!}: \operatorname{D}-\bmod \left(y_{1}\right) \rightarrow \operatorname{Pro}\left(\mathrm{D}-\bmod \left(y_{2}\right)\right) \text { and } f^{*}: \operatorname{D}-\bmod \left(y_{2}\right) \rightarrow \operatorname{Pro}\left(\mathrm{D}-\bmod \left(y_{1}\right)\right) .
$$

4.2.2. Let $Z, Z^{0}, Z^{ \pm}, p^{ \pm}, i^{ \pm}$be as in Sect. 2.3.1

Consider the corresponding commutative (but not necessarily Cartesian) diagram

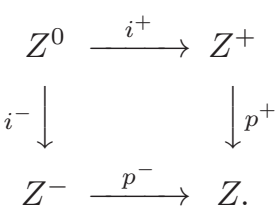

We enlarge it to the diagram

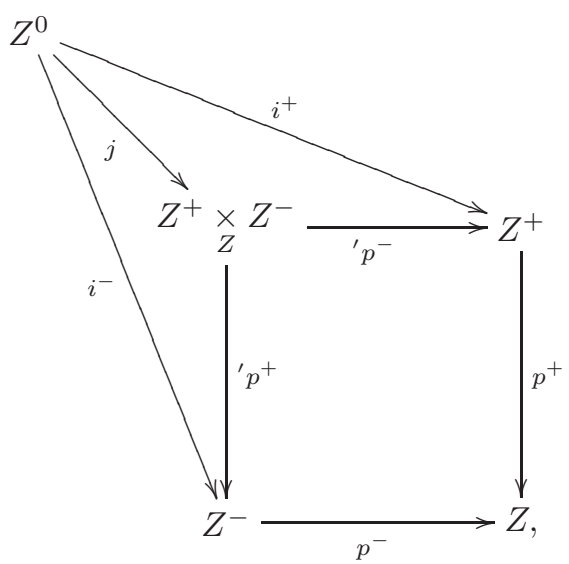

in which the square is Cartesian, and where (according to [DrGa3, Proposition 1.9.4]) the map

$$
j: Z^{0} \rightarrow Z_{Z}^{+} \underset{Z}{\times} Z^{-}
$$


is an open embedding.

We consider the categories

$$
\operatorname{D-mod}(Z)^{\mathbb{G}_{m}-\operatorname{mon}}, \operatorname{D}-\bmod \left(Z^{+}\right)^{\mathbb{G}_{m}-\operatorname{mon}}, \operatorname{D-mod}\left(Z^{-}\right)^{\mathbb{G}_{m}-\operatorname{mon}}
$$

and

Consider the functors

$$
\operatorname{D}-\bmod \left(Z^{0}\right)^{\mathbb{G}_{m}-\operatorname{mon}}=\operatorname{D}-\bmod \left(Z^{0}\right)
$$

$\left(p^{+}\right)^{!}: \mathrm{D}-\bmod (Z)^{\mathbb{G}_{m}-\operatorname{mon}} \rightarrow \mathrm{D}-\bmod \left(Z^{+}\right)^{\mathbb{G}_{m}-\operatorname{mon}}$ and $\left(i^{-}\right)^{!}: \mathrm{D}-\bmod \left(Z^{-}\right)^{\mathbb{G}_{m}-\operatorname{mon}} \rightarrow \mathrm{D}-\bmod \left(Z^{0}\right)$.

Consider also the functors

and

$$
\left(p^{-}\right)^{*}: \operatorname{D}-\bmod (Z)^{\mathbb{G}_{m}-\operatorname{mon}} \rightarrow \operatorname{Pro}\left(\mathrm{D}-\bmod \left(Z^{-}\right)^{\mathbb{G}_{m}-\operatorname{mon}}\right)
$$

$\left(i^{+}\right)^{*}: \operatorname{D}-\bmod \left(Z^{+}\right)^{\mathbb{G}_{m}-\operatorname{mon}} \rightarrow \operatorname{Pro}\left(\operatorname{D}-\bmod \left(Z^{0}\right)\right)$,

left adjoint in the sense of [DrGa3, Sect. A.3] to

$$
\left(p^{-}\right)_{*}: \mathrm{D}-\bmod \left(Z^{-}\right)^{\mathbb{G}_{m}-\operatorname{mon}} \rightarrow \mathrm{D}-\bmod (Z)^{\mathbb{G}_{m}-\operatorname{mon}}
$$

and

respectively.

$$
\left(i^{+}\right)_{*}: \mathrm{D}-\bmod \left(Z^{0}\right) \rightarrow \mathrm{D}-\bmod \left(Z^{+}\right)^{\mathbb{G}_{m}-\operatorname{mon}}
$$

4.2.3. Consider the composed functors

$$
\left(i^{+}\right)^{*} \circ\left(p^{+}\right)^{!} \text {and }\left(i^{-}\right)^{!} \circ\left(p^{-}\right)^{*}, \quad \operatorname{D}-\bmod (Z)^{\mathbb{G}_{m}-\operatorname{mon}} \rightarrow \operatorname{Pro}\left(\mathrm{D}-\bmod \left(Z^{0}\right)\right) .
$$

They are called the functors of hyperbolic restriction.

We note that there is a canonical natural transformation

$$
\left(i^{+}\right)^{*} \circ\left(p^{+}\right)^{!} \rightarrow\left(i^{-}\right)^{!} \circ\left(p^{-}\right)^{*} .
$$

Namely, the natural transformation (4.4) is obtained via the $\left(\left(i^{+}\right)^{*},\left(i^{+}\right)_{*}\right)$-adjunction from the natural transformation

$$
\left(p^{+}\right)^{!} \rightarrow\left(i^{+}\right)_{*} \circ\left(i^{-}\right)^{!} \circ\left(p^{-}\right)^{*},
$$

defined in terms of (4.3) as follows:

$$
\begin{aligned}
\left(p^{+}\right)^{!} \rightarrow\left(p^{+}\right)^{!} \circ\left(p^{-}\right)_{*} \circ\left(p^{-}\right)^{*} \simeq\left({ }^{\prime} p^{-}\right)_{*} \circ\left({ }^{\prime} p^{+}\right)^{!} \circ\left(p^{-}\right)^{*} \rightarrow & \\
& \rightarrow\left({ }^{\prime} p^{-}\right)_{*} \circ j_{*} \circ j^{!} \circ\left({ }^{\prime} p^{+}\right)^{!} \circ\left(p^{-}\right)^{*} \simeq\left(i^{+}\right)_{*} \circ\left(i^{-}\right)^{!} \circ\left(p^{-}\right)^{*},
\end{aligned}
$$

where the map

$$
\mathrm{Id} \rightarrow j_{*} \circ j^{!}
$$

comes from the $\left(j^{1}, j_{*}\right)$-adjunction using the fact that $j$ is an open embedding, and where $\left(p^{+}\right)^{!} \circ\left(p^{-}\right)_{*} \simeq\left({ }^{\prime} p^{-}\right)_{*} \circ\left({ }^{\prime} p^{+}\right)^{!}$is the base change isomorphism.

4.2.4. Braden's theorem of $[\mathrm{Br}$ ( as stated in $[\mathrm{DrGa3}$, Theorem 3.1.6]) reads:

Theorem 4.2.5. The functors

$$
\left(i^{+}\right)^{*} \circ\left(p^{+}\right)^{!} \text {and }\left(i^{-}\right)^{!} \circ\left(p^{-}\right)^{*}, \quad \operatorname{D}-\bmod (Z)^{\mathbb{G}_{m}-\operatorname{mon}} \rightarrow \operatorname{Pro}\left(\operatorname{D}-\bmod \left(Z^{0}\right)\right)
$$

take values in $\mathrm{D}-\bmod \left(Z^{0}\right) \subset \operatorname{Pro}\left(\mathrm{D}-\bmod \left(Z^{0}\right)\right)$ and the map (4.4) is an isomophism.

\subsection{The setting for stacks.}


4.3.1. Assume now that we are given a commuttaive diagram of algebraic stacks:

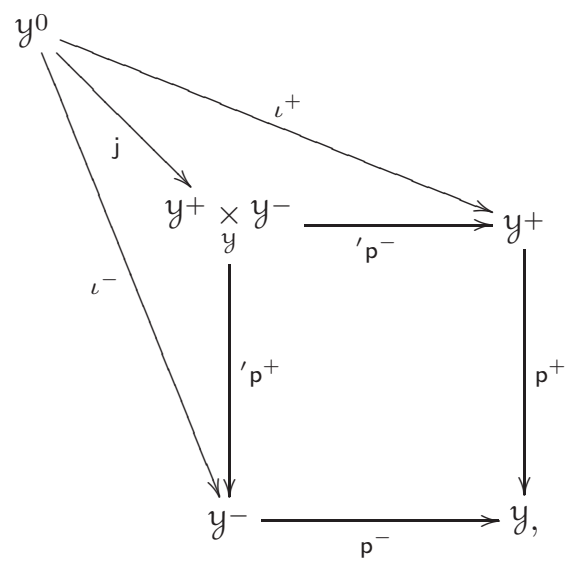

where the square is Cartesian, and the map $j: y^{0} \rightarrow y^{+} \underset{y}{x} y^{-}$is an open embedding.

We will assume that all morphisms in (4.5) are quasi-compact and safe.

Consider the functors

$$
\left(\iota^{-}\right)^{*} \circ\left(\mathrm{p}^{-}\right)^{!} \text {and }\left(\iota^{+}\right)^{!} \circ\left(\mathrm{p}^{+}\right)^{*}: \mathrm{D}-\bmod (\mathrm{y}) \rightarrow \operatorname{Pro}\left(\mathrm{D}-\bmod \left(\mathrm{y}^{0}\right)\right) .
$$

As in the case of (4.4), we obtain a natural transformation

$$
\left(\iota^{-}\right)^{*} \circ\left(\mathrm{p}^{-}\right)^{!} \rightarrow\left(\iota^{+}\right)^{!} \circ\left(\mathrm{p}^{+}\right)^{*} .
$$

4.3.2. Definition of hyperbolicity for stacks. We will say that the diagram (4.5) is hyperbolic if the functors $\left(\iota^{-}\right)^{*} \circ\left(\mathrm{p}^{-}\right)^{!}$and $\left(\iota^{+}\right)^{!} \circ\left(\mathrm{p}^{+}\right)^{*}$ take values in

$$
\mathrm{D}-\bmod \left(\mathrm{y}^{0}\right) \subset \operatorname{Pro}\left(\mathrm{D}-\bmod \left(\mathrm{y}^{0}\right)\right),
$$

and the map (4.6) is an isomorphism.

4.3.3. Checking hyperbolicity. Let $Z$ be again as in Sect. 2.3.1. Let us be given a commutative cube

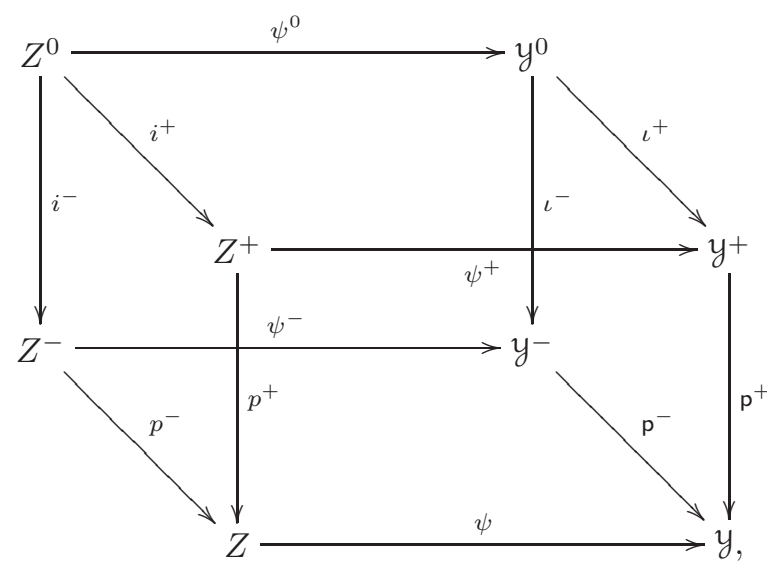

where the maps

$$
\psi: Z \rightarrow y, \psi^{+}: Z^{+} \rightarrow y^{+}, \psi^{-}: Z^{-} \rightarrow y^{-}, \psi^{0}: Z^{0} \rightarrow y^{0}
$$


are smooth, and $\psi^{0}$ surective. Assume also that the morphism $\psi: Z \rightarrow y$ can be given a $\mathbb{G}_{m}$-equivariant structure (with respect to the trivial action of $\mathbb{G}_{m}$ on $y$ ).

We claim:

Theorem 4.3.4. Under the above circumstances the diagram (4.5) is hyperbolic.

The proof is given in Appendix $\mathrm{A}$.

\subsection{Hyperbolocity and adjunction.}

4.4.1. Assume that in the situation of diagram (4.5), the morphisms $\iota^{-}$and $\iota^{+}$in (4.5) admit left inverses, denoted $\mathrm{q}^{-}$and $\mathrm{q}^{+}$, respectively. Assume also that the morphisms $\mathrm{q}^{ \pm}$are quasicompact.

The diagram

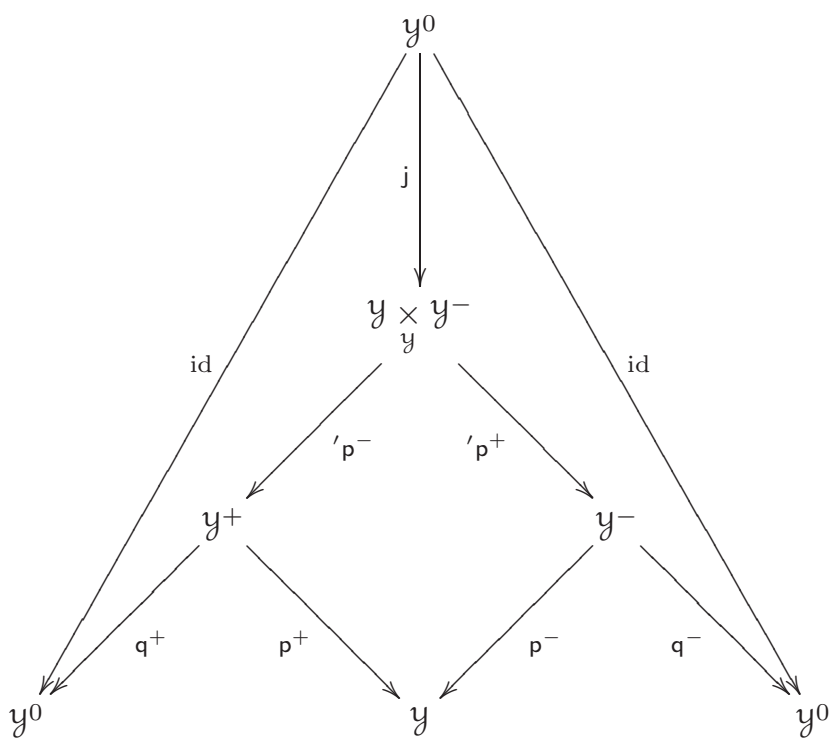

gives rise to a natural transformation

$$
\left(\mathrm{q}^{-}\right)_{\mathbf{\Delta}} \circ\left(\mathrm{p}^{-}\right)^{!} \circ\left(\mathrm{p}^{+}\right)_{*} \circ\left(\mathrm{q}^{+}\right)^{!} \simeq\left(\mathrm{q}^{-}\right)_{\mathbf{\Delta}} \circ\left(\mathrm{p}^{-}\right)^{!} \circ\left(\mathrm{p}^{+}\right)_{\mathbf{\Delta}} \circ\left(\mathrm{q}^{+}\right)^{!} \rightarrow \operatorname{Id}_{\mathrm{D}-\bmod \left(y^{\circ}\right)},
$$

which by adjunction gives rise to a natural transformation

$$
\left(\mathrm{q}^{-}\right) \mathbf{\Delta} \circ\left(\mathrm{p}^{-}\right)^{!} \rightarrow\left(\mathrm{q}^{+}\right) ! \circ\left(\mathrm{p}^{+}\right)^{*}, \quad \mathrm{D}-\bmod (\mathrm{y}) \rightarrow \operatorname{Pro}\left(\mathrm{D}-\bmod \left(\mathrm{y}^{0}\right)\right) .
$$

4.4.2. Assume now that the natural transformation

$$
\left(\mathrm{q}^{-}\right)_{\mathbf{\Delta}} \circ\left(\iota^{-}\right)_{*} \simeq\left(\mathrm{q}^{-}\right)_{\mathbf{\Delta}} \circ\left(\iota^{-}\right)_{\mathbf{\Delta}} \stackrel{\sim}{\rightarrow} \operatorname{Id}_{\mathrm{D}-\bmod \left(y^{0}\right)}
$$

identifies the functor $\left(\mathrm{q}^{-}\right)_{\mathbf{\Delta}}$ with the left adjoint $\left(\iota^{-}\right)^{*}$ of $\left(\iota^{-}\right)_{*}$.

Assume also that the natural transformation

$$
\left(\iota^{+}\right)^{!} \circ\left(\mathrm{q}^{+}\right)^{!} \stackrel{\sim}{\rightarrow} \operatorname{Id}_{\mathrm{D}-\bmod \left(y^{0}\right)}
$$

identifies the functor $\left(\iota^{+}\right)^{!}$with the left adjoint $\left(\mathrm{q}^{+}\right)$! of $\left(\mathrm{q}^{+}\right)^{\text {! }}$.

In particular, both of the above left adjoints take values in

$$
\operatorname{D}-\bmod \left(y^{0}\right) \subset \operatorname{Pro}\left(\operatorname{D}-\bmod \left(y^{0}\right)\right) \text {. }
$$


(For example, such behavior of the morphisms $\left(\mathrm{q}^{-}, \imath^{-}\right)$and $\left(\mathrm{q}^{+}, \imath^{+}\right)$occurs in the situation described in Sect. 4.1.2)

In this case diagram chase shows that the following diagram of natural transformations commutes:

From here we obtain:

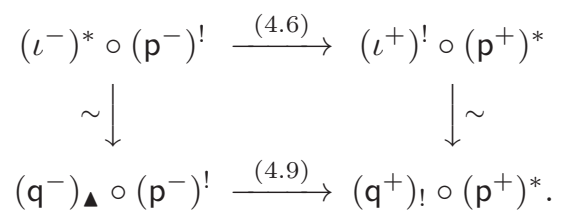

Lemma 4.4.3. The diagram (4.5) is hyperbolic if and only if the natural transformation

$$
\left(\left(\mathrm{q}^{-}\right)_{\mathbf{\Delta}} \circ\left(\mathrm{p}^{-}\right)^{!}\right) \circ\left(\left(\mathrm{p}^{+}\right)_{*} \circ\left(\mathrm{q}^{+}\right)^{!}\right) \rightarrow \operatorname{Id}_{\mathrm{D}-\bmod \left(y^{\circ}\right)}
$$

of (4.8) defines the co-unit of an adjunction, making the functor $\left(\mathrm{q}^{-}\right) \mathbf{\Delta} \circ\left(\mathrm{p}^{-}\right)^{!}$into a left adjoint of $\left(\mathrm{p}^{+}\right)_{*} \circ\left(\mathrm{q}^{+}\right)^{!}$.

4.4.4. Note that Lemma 4.4.3, combined with Proposition 4.1.5, imply that the assertion of Theorem 1.2.5 (with the co-unit specified in Sect. 1.3.1) is equivalent to the following:

Corollary 4.4.5. The diagram

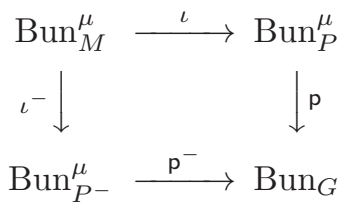

is hyperbolic.

\subsection{Proof of Theorem 1.2.5 reduction to the quasi-compact case.}

4.5.1. We need to show that for $\mathcal{F}_{G} \in \mathrm{D}-\bmod \left(\operatorname{Bun}_{G}\right)$ and $\mathcal{F}_{M} \in \mathrm{D}-\bmod \left(\operatorname{Bun}_{M}^{\mu}\right)$, the map

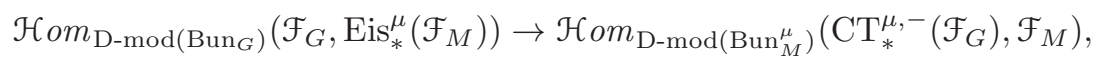

induced by (1.4), is an isomorphism.

In this subsection we will show that it is sufficient to prove that (4.10) is an isomorphism for any $\mathcal{F}_{M}$ of the form $\left(\jmath_{M}\right)_{*}\left(\mathcal{F}_{M}^{\prime}\right)$, where

$$
U_{M} \stackrel{\jmath M}{\longrightarrow} \operatorname{Bun}_{M}^{\mu}
$$

is an open quasi-compact substack and $\mathscr{F}_{M}^{\prime} \in \mathrm{D}-\bmod \left(U_{M}\right)$.

4.5.2. Let open-qc ${ }_{G}$ denote the poset of open quasi-compact substacks of $\operatorname{Bun}_{G}$, and let open-qc ${ }_{M}$ be the corresponding poset for $\operatorname{Bun}_{M}^{\mu}$.

For every

$$
\left(U_{M} \stackrel{J_{M}}{\longrightarrow} \operatorname{Bun}_{M}^{\mu}\right) \in \text { open-qc }_{M}
$$

we have a commutative diagram

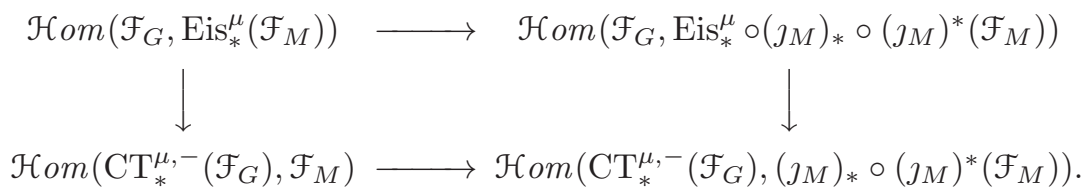


Hence, it suffices to show that the maps

$$
\begin{aligned}
\mathcal{H}_{\text {om }_{\mathrm{D}-\bmod \left(\operatorname{Bun}_{M}\right)}}\left(\mathrm{CT}_{*}^{\mu,-}\left(\mathcal{F}_{G}\right), \mathcal{F}_{M}\right) \rightarrow \\
\rightarrow \underset{U_{M} \in \mathrm{open}-\mathrm{qc}_{M}}{\lim } \mathcal{H} \operatorname{lom}_{\mathrm{D}-\bmod \left(\operatorname{Bun}_{M}\right)}\left(\mathrm{CT}_{*}^{\mu,-}\left(\mathcal{F}_{G}\right),\left(\jmath_{M}\right)_{*} \circ\left(\jmath_{M}\right)^{*}\left(\mathcal{F}_{M}\right)\right)
\end{aligned}
$$

and

$$
\begin{aligned}
& \mathcal{H} m_{\mathrm{D}-\bmod \left(\operatorname{Bun}_{G}\right)}\left(\mathcal{F}_{G}, \operatorname{Eis}_{*}^{\mu}\left(\mathcal{F}_{M}\right)\right) \rightarrow \\
& \rightarrow \underset{U_{M} \in \text { open-qc }_{M}}{\lim } \mathcal{H} \text { om }_{\mathrm{D}-\bmod \left(\operatorname{Bun}_{G}\right)}\left(\mathcal{F}_{G}, \operatorname{Eis}_{*}^{\mu} \circ\left(\jmath_{M}\right)_{*} \circ\left(\jmath_{M}\right)^{*}\left(\mathcal{F}_{M}\right)\right)
\end{aligned}
$$

are isomorphisms.

4.5.3. The fact that (4.11) is an isomorphism is evident from the definition of $D-\bmod \left(\operatorname{Bun}_{M}\right)$ as

$$
\underset{U_{M} \in \text { open-qc }_{M}}{\lim } \operatorname{D-mod}\left(U_{M}\right)
$$

since

$$
\begin{aligned}
& \mathcal{H} \text { om }_{\mathrm{D}-\bmod \left(\operatorname{Bun}_{M}\right)}\left(\mathrm{CT}_{*}^{\mu,-}\left(\mathcal{F}_{G}\right),\left(\jmath_{M}\right)_{*} \circ\left(\jmath_{M}\right)^{*}\left(\mathcal{F}_{M}\right)\right) \simeq \\
& \simeq \mathcal{H} \text { om }_{\mathrm{D}-\bmod \left(U_{M}\right)}\left(\left(\jmath_{M}\right)^{*} \circ \mathrm{CT}_{*}^{\mu,-}\left(\mathcal{F}_{G}\right),\left(\jmath_{M}\right)^{*}\left(\mathcal{F}_{M}\right)\right) \text {. }
\end{aligned}
$$

Similarly, the map

$$
\begin{aligned}
& \mathcal{H} \operatorname{om}_{\mathrm{D}-\bmod \left(\operatorname{Bun}_{G}\right)}\left(\mathcal{F}_{G}, \operatorname{Eis}_{*}^{\mu}\left(\mathcal{F}_{M}\right)\right) \rightarrow
\end{aligned}
$$

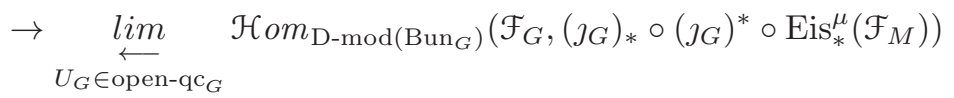

is an isomorphism.

4.5.4. Note now that we have a commutative diagram

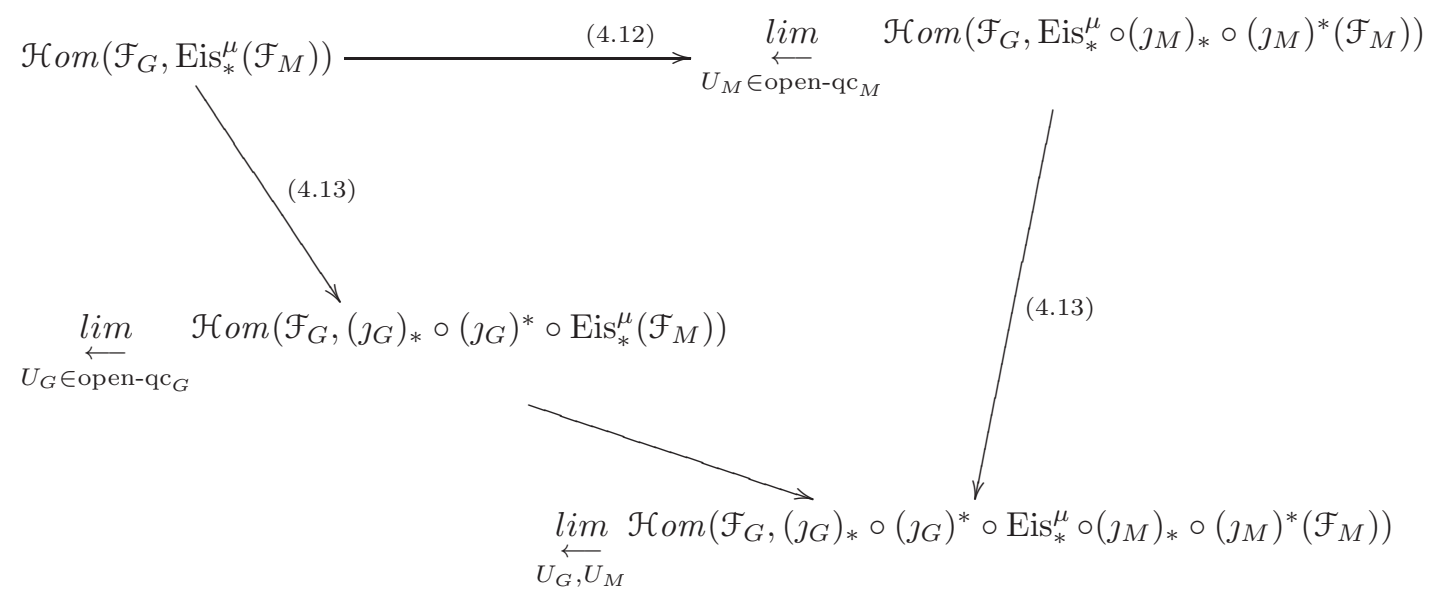


It remains to show that the map

$$
\begin{aligned}
\lim _{U_{G} \in \text { open-qc }_{G}} \mathcal{H} \operatorname{Hom}\left(\mathcal{F}_{G},\left(\jmath_{G}\right)_{*} \circ\left(\jmath_{G}\right)^{*} \circ \operatorname{Eis}_{*}^{\mu}\left(\mathcal{F}_{M}\right)\right) \rightarrow & \\
& \rightarrow \underset{U_{G}, U_{M}}{\lim } \mathcal{H} \mathcal{H o m}\left(\mathcal{F}_{G},\left(\jmath_{G}\right)_{*} \circ\left(\jmath_{G}\right)^{*} \circ \operatorname{Eis}_{*}^{\mu} \circ\left(\jmath_{M}\right)_{*} \circ\left(\jmath_{M}\right)^{*}\left(\mathcal{F}_{M}\right)\right)
\end{aligned}
$$

is an isomorphism.

However, we claim that for any fixed $U_{G}$, the corresponding map

$$
\begin{aligned}
\mathcal{H} o m\left(\mathcal{F}_{G},\left(\jmath_{G}\right)_{*} \circ\left(\jmath_{G}\right)^{*} \circ \operatorname{Eis}_{*}^{\mu}\left(\mathcal{F}_{M}\right)\right) \rightarrow & \rightarrow \underset{U_{M} \in \text { open-qc }_{M}}{\stackrel{\lim }{H}} \mathcal{H o m}\left(\mathcal{F}_{G},\left(\jmath_{G}\right)_{*} \circ\left(\jmath_{G}\right)^{*} \circ \operatorname{Eis}_{*}^{\mu} \circ\left(\jmath_{M}\right)_{*} \circ\left(\jmath_{M}\right)^{*}\left(\mathcal{F}_{M}\right)\right)
\end{aligned}
$$

is already an isomorphism. In fact, the map

$$
\left(\jmath_{G}\right)^{*} \circ \operatorname{Eis}_{*}^{\mu}\left(\mathcal{F}_{M}\right) \rightarrow\left(\jmath_{G}\right)^{*} \circ \operatorname{Eis}_{*}^{\mu} \circ\left(\jmath_{M}\right)_{*} \circ\left(\jmath_{M}\right)^{*}\left(\mathcal{F}_{M}\right)
$$

is an isomorphism, whenever $U_{M}$ is such that

$$
\mathrm{q}\left(\mathrm{p}^{-1}\left(U_{G}\right) \subset U_{M},\right.
$$

and such $U_{M}$ are cofinal in open-qc ${ }_{M}$.

\subsection{Proof of Theorem 1.2.5 reduction to the question of hyperbolicity.}

4.6.1. According to Sect. 4.5, it suffices to show that for an open quasi-compact substack

$$
U_{M} \stackrel{J_{M}}{\longrightarrow} \operatorname{Bun}_{M}^{\mu},
$$

the natural transformation

$$
\jmath_{M}^{*} \circ \mathrm{CT}_{*}^{\mu,-} \circ \operatorname{Eis}_{*}^{\mu} \circ\left(\jmath_{M}\right)_{*} \stackrel{1.4}{\longrightarrow} \jmath_{M}^{*} \circ\left(\jmath_{M}\right)_{*} \rightarrow \operatorname{Id}_{\mathrm{D}-\bmod \left(U_{M}\right)}
$$

defines the co-unit of an adjunction.

4.6.2. Let $U_{G} \subset \operatorname{Bun}_{G}$ be a quasi-compact open substack such that

$$
\mathrm{p}\left(\mathrm{q}^{-1}\left(U_{M}\right)\right) \subset U_{G} \text { and } \mathrm{p}^{-}\left(\left(\mathrm{q}^{-}\right)^{-1}\left(U_{M}\right)\right) \subset U_{G} .
$$

Consider the corresponding diagram

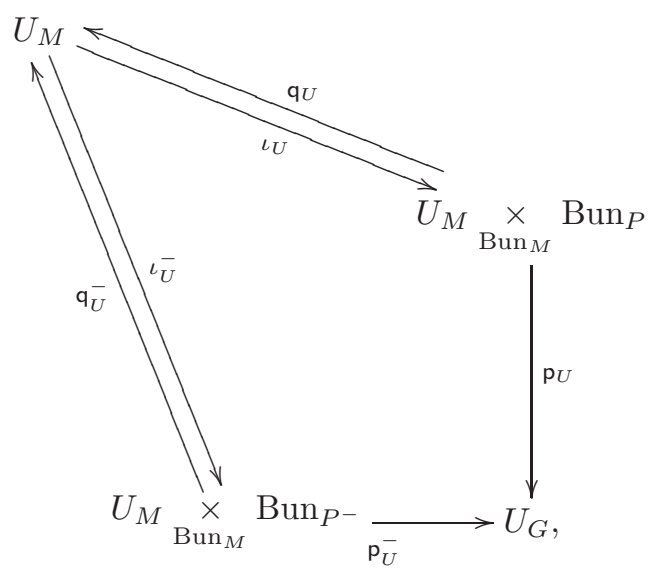


It suffices to show that the functor

$$
\left(\mathrm{q}_{U}\right) ! \circ \mathrm{p}_{U}^{*}: \mathrm{D}-\bmod \left(U_{G}\right) \rightarrow \operatorname{Pro}\left(\mathrm{D}-\bmod \left(U_{M}\right)\right)
$$

takes values in $\mathrm{D}-\bmod \left(U_{M}\right)$, and that the natural transformation

$$
\left(\mathrm{q}_{U}^{-}\right)_{*} \circ\left(\mathrm{p}_{U}^{-}\right)^{!} \rightarrow\left(\mathrm{q}_{U}\right) ! \circ\left(\mathrm{p}_{U}\right)^{*}
$$

of (4.9) is an isomorphism.

4.6.3. The contraction of $\operatorname{Bun}_{P}$ (resp., Bun $P^{-}$) onto $\operatorname{Bun}_{M}$ (see Sect. 4.1.6) and Proposition 4.1.5 imply that the morphisms

$$
\mathbf{q}_{U}: U_{M} \underset{\operatorname{Bun}_{M}}{\times} \operatorname{Bun}_{P} \rightleftarrows U_{M}: \iota_{U}
$$

and

$$
\mathbf{q}_{U}^{-}: U_{M} \underset{\operatorname{Bun}_{M}}{\times} \operatorname{Bun}_{P^{-}} \rightleftarrows U_{M}: \iota_{U}^{-}
$$

satisfy the assumptions of Sect. 4.4.2

Hence, applying Lemma 4.4.3 we obtain that it is enough to show that the diagram

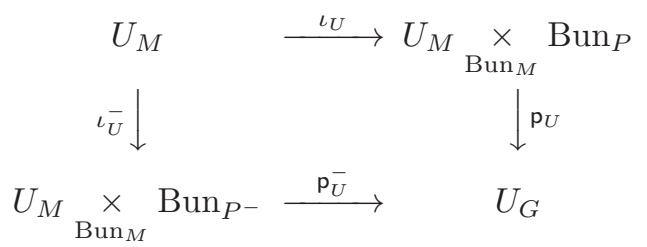

is hyperbolic in the sense of Sect. 4.3.2.

\subsection{Proof of Theorem 1.2.5 verification of hyperbolicity.}

4.7.1. Fix any point $x \in X$. For $H=G, P, P^{-}$or $M$, let $\operatorname{Bun}_{H}^{n \cdot x}$ be the stack classifying $G$-bundles with a structure of level $n$ at $x$. It is known that for $n$ large enough, the open substacks

$$
\begin{aligned}
& U_{G} \underset{\operatorname{Bun}_{G}}{\times} \operatorname{Bun}_{G}^{n \cdot x} \subset \operatorname{Bun}_{G}^{n \cdot x} \\
& U_{M} \underset{\operatorname{Bun}_{M}}{\times} \operatorname{Bun}_{M}^{n \cdot x} \subset \operatorname{Bun}_{M}^{n \cdot x}, \\
& U_{M} \underset{\operatorname{Bun}_{M}}{\times} \operatorname{Bun}_{P}^{n \cdot x} \simeq\left(U_{M} \underset{\operatorname{Bun}_{M}}{\times} \operatorname{Bun}_{P}\right) \underset{\operatorname{Bun}_{P}}{\times} \operatorname{Bun}_{P}^{n \cdot x}
\end{aligned}
$$

and

$$
U_{M} \underset{\operatorname{Bun}_{M}}{\times} \operatorname{Bun}_{P^{-}}^{n \cdot x} \simeq\left(U_{M} \underset{\operatorname{Bun}_{M}}{\times} \operatorname{Bun}_{P^{-}}\right) \underset{\operatorname{Bun}_{P^{-}}}{\times} \operatorname{Bun}_{P^{-}}^{n \cdot x}
$$

are actually quasi-compact separated schemes.

By making $n$ even larger, we can assume that the maps in the diagram

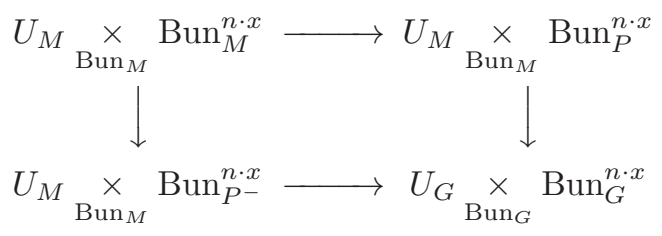

are locally closed embeddings.

Combining, we obtain the following diagram: 


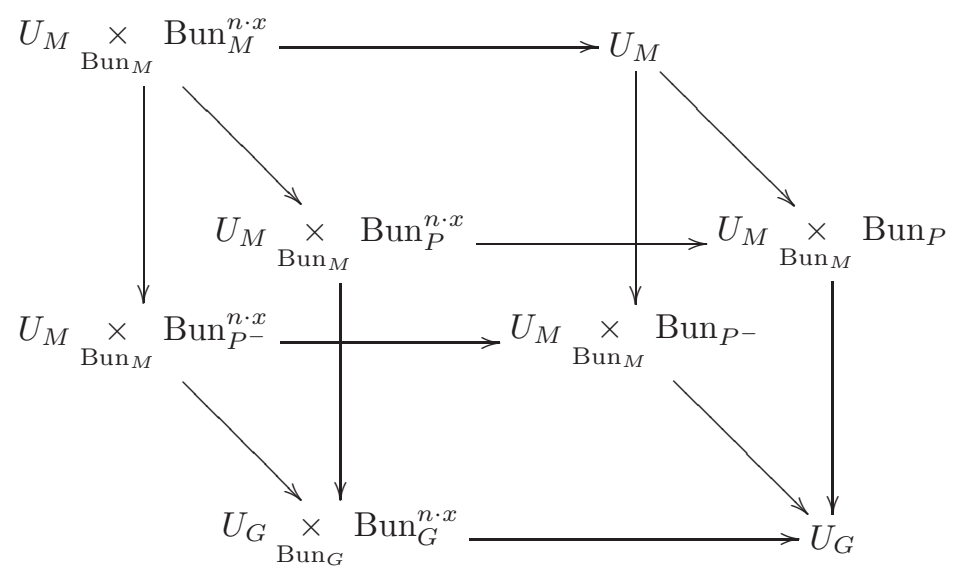

4.7.2. Let $\gamma: \mathbb{G}_{m} \rightarrow M$ be a co-character as in (2.13), and consider the corresponding homomorphism

$$
\mathbb{G}_{m} \rightarrow H .
$$

The adjoint action of $\mathbb{G}_{m}$ on $H$ defines a $\mathbb{G}_{m}$-action on each Bun ${ }_{H}^{n \cdot x}$ so that the forgetful maps

$$
\operatorname{Bun}_{H}^{n \cdot x} \rightarrow \operatorname{Bun}_{H}
$$

are $\mathbb{G}_{m}$-equivariant. In particular, the scheme

$$
Z:=U_{G} \underset{\operatorname{Bun}_{G}}{\times} \operatorname{Bun}_{G}^{n \cdot x}
$$

acquires a $\mathbb{G}_{m}$-action, which is locally linear, i.e., $Z$ can be covered by affine subschemes that are preserved by the $\mathbb{G}_{m}$-action.

Consider the corresponding diagram of (4.2):

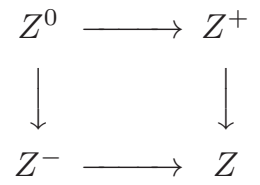

for $Z$ as above.

By Theorem 4.3.4 in order to show that the diagram (4.15) is hyperbolic, it is enough to show that the digram (4.16) admits a map to the diagram (4.18), which is an open embedding on a Zariski neighborhood of $U_{M} \underset{\operatorname{Bun}_{M}}{\times} \operatorname{Bun}_{M}^{n \cdot x}$. (Indeed, in this case the diagram (4.16) will identify with the diagram (4.2) for an open $\mathbb{G}_{m}$-invariant subscheme of $Z$.)

4.7.3. We construct the corresponding maps

$$
\begin{aligned}
& U_{M} \underset{\operatorname{Bun}_{M}}{\times} \operatorname{Bun}_{M}^{n \cdot x} \rightarrow Z^{0}, \\
& U_{M} \underset{\operatorname{Bun}_{M}}{\times} \operatorname{Bun}_{P}^{n \cdot x} \rightarrow Z^{+},
\end{aligned}
$$

and

$$
U_{M} \underset{\operatorname{Bun}_{M}}{\times} \operatorname{Bun}_{P^{-}}^{n \cdot x} \rightarrow Z^{0}
$$

as follows. 
The map (4.19) is evident, since the adjoint action of $\mathbb{G}_{m}$ on $M$ is trivial.

The map (4.20) (resp., (4.21) ) is given by the $\mathbb{A}^{1}$-action on $\operatorname{Bun}_{P}^{n \cdot x}$ (resp., Bun $P^{n-x}$ ) that contracts it onto $\operatorname{Bun}_{M}^{n \cdot x}$ as in Sect. 4.1.6.

4.7.4. The map (4.19) (resp., (4.20), (4.21) is a locally closed embedding, because its composition with the map $Z^{0} \rightarrow Z$ (resp., $Z^{+} \rightarrow Z, Z^{-} \rightarrow Z$ ) is.

It remains to show that the maps (4.19), 4.20) and 4.21) are open embeddings on a Zariski neighborhood of $U_{M} \underset{\operatorname{Bun}_{M}}{\times} \operatorname{Bun}_{M}^{n \cdot x}$.

Since the left-hand sides are smooth, it is enough to show that maps in question give rise to isomorphisms of tangent spaces at every $k$-point of $U_{M} \underset{\operatorname{Bun}_{M}}{\times} \operatorname{Bun}_{M}^{n \cdot x}$.

Let $z$ be a $k$-point of $Z$, where $Z$ is as in Sect. 4.2. The $\mathbb{G}_{m}$-action on $Z$ induces a linear action of $\mathbb{G}_{m}$ on the tangent space $T_{z}(Z)$. It is easy to see that the subspaces

$$
T_{z}\left(Z^{0}\right), T_{z}\left(Z^{+}\right) \text {and } T_{z}\left(Z^{-}\right)
$$

identify, respectively, with the subspace of zero, non-negative and non-positive characters of $\mathbb{G}_{m}$ on $T_{z}(Z)$.

Let $Z:=U_{G} \underset{\operatorname{Bun}_{G}}{\times} \operatorname{Bun}_{G}^{n \cdot x}$, and let $z$ be a geometric point of $U_{M} \underset{\operatorname{Bun}_{M}}{\times} \operatorname{Bun}_{M}^{n \cdot x}$, corresponding to an $M$-bundle $\mathcal{F}_{M}$.

The tangent space $T_{z}\left(U_{G} \underset{\operatorname{Bun}_{G}}{\times} \operatorname{Bun}_{G}^{n \cdot x}\right)$ identifies with $H^{1}\left(X, \mathfrak{g}_{\mathcal{F}_{M}}(-n \cdot x)\right)$, while the tangent spaces

$$
T_{z}\left(U_{M} \underset{\operatorname{Bun}_{M}}{\times} \operatorname{Bun}_{M}^{n \cdot x}\right), T_{z}\left(U_{M} \underset{\operatorname{Bun}_{M}}{\times} \operatorname{Bun}_{P}^{n \cdot x}\right) \text { and } T_{z}\left(U_{M} \underset{\operatorname{Bun}_{M}}{\times} \operatorname{Bun}_{P^{-}}^{n \cdot x}\right)
$$

identify with

$$
H^{1}\left(X, \mathfrak{m}_{\mathcal{F}_{M}}(-n \cdot x)\right), H^{1}\left(X, \mathfrak{p}_{\mathcal{F}_{M}}(-n \cdot x)\right) \text { and } H^{1}\left(X, \mathfrak{p}_{\mathcal{F}_{M}}(-n \cdot x)\right),
$$

respectvely.

This makes the required assertion manifest.

\section{Appendix A. Hyperbolicity For stacks: proof of Theorem 4.3.4}

With no restriction of generality, we can assume that $y$ is quasi-compact (and hence QCA). Indeed, otherwise, replace it by the image of the map $\psi$.

A.1. Step 1. In this subsection we will show that the functors

$$
\left(\iota^{-}\right)^{*} \circ\left(\mathrm{p}^{-}\right)^{!} \text {and }\left(\iota^{+}\right)^{*} \circ\left(\mathrm{p}^{+}\right)^{!}
$$

take values in $D-\bmod \left(y^{0}\right) \subset \operatorname{Pro}\left(D-\bmod \left(y^{0}\right)\right)$. By symmetry, it is sufficient to treat the case of the former functor. 
A.1.1. Note that for $\mathcal{F} \in \mathrm{D}-\bmod (y)$, the object

$$
\left(\psi^{0}\right)^{*} \circ\left(\iota^{-}\right)^{*} \circ\left(\mathrm{p}^{-}\right)^{!}(\mathcal{F}),
$$

is, isomorphic to $\left(i^{-}\right)^{*} \circ\left(\psi^{-}\right)^{*} \circ\left(\mathrm{p}^{-}\right)^{!}(\mathcal{F})$ and the latter is, up to a cohomological shift, isomorphic to $\left(i^{-}\right)^{*} \circ\left(p^{-}\right)^{!} \circ \psi^{*}(\mathcal{F})$, since the morphisms $\psi$ and $\psi^{-}$are both smooth.

Since $\psi^{*}(\mathcal{F}) \in \mathrm{D}-\bmod (Z)^{\mathbb{G}_{m}-\operatorname{mon}}$, from Theorem 4.2.5, we obtain that

$$
\left(i^{-}\right)^{*} \circ\left(p^{-}\right)^{!} \circ \psi^{*}(\mathcal{F}) \in \mathrm{D}-\bmod \left(Z^{0}\right) .
$$

Hence, we obtain that

$$
\left(\psi^{0}\right)^{*} \circ\left(\iota^{-}\right)^{*} \circ\left(\mathrm{p}^{-}\right)^{!}(\mathcal{F}) \in \mathrm{D}-\bmod \left(Z^{0}\right) .
$$

Now, the fact that $\left(\iota^{-}\right)^{*} \circ\left(\mathrm{p}^{-}\right)^{!}(\mathcal{F})$ belongs to $\mathrm{D}-\bmod \left(\mathrm{y}^{0}\right)$ follows from the next general assertion:

Lemma A.1.2. Let $z \stackrel{g}{\longrightarrow} y_{1} \stackrel{f}{\longrightarrow} y_{2}$ be maps between quasi-compact stacks with $g$ smooth and surjective. Then for $\mathcal{F} \in \mathrm{D}-\bmod \left(y_{2}\right)$, the object $f^{*}(\mathcal{F})$ belongs to $\mathrm{D}-\bmod \left(y_{1}\right)$ if and only if $g^{*} \circ f^{*}(\mathcal{F})$ belongs to $\mathrm{D}-\bmod (\mathcal{Z})$.

A.1.3. Proof of Lemma A.1.2. The "only" if direction is obvious. For the "if" direction, with no restriction of generality, we can assume that $z$ is a quasi-compact scheme.

Let $z^{\bullet}$ denote the Čech nerve of the map $g$, and let $g^{\bullet}: z^{\bullet} \rightarrow y_{1}$ denote the corresponding maps. By assumption $\left(g^{\bullet}\right)^{*} \circ f^{*}(\mathcal{F})$ is a well-defined an object of $\operatorname{Tot}\left(\operatorname{D}-\bmod \left(z^{\bullet}\right)\right)$. Since $g$ is smooth and surjective, the functor

$$
\left(g^{\bullet}\right)^{*}: \mathrm{D}-\bmod \left(y_{1}\right) \rightarrow \operatorname{Tot}\left(\mathrm{D}-\bmod \left(z^{\bullet}\right)\right)
$$

is an equivalence. Let $\mathcal{F}^{\prime} \in \mathrm{D}$ - $\bmod \left(y_{1}\right)$ denote the object corresponding to $\left(g^{\bullet}\right)^{*} \circ f^{*}(\mathcal{F})$ under the equivalence (A.2). We claim that $\mathcal{F}^{\prime} \simeq f^{*}(\mathcal{F})$.

It suffices to show that for $\mathcal{F}_{1} \in \mathrm{D}$-mod$\left(y_{1}\right)$, we have a canonical ismorphism

$$
\mathcal{H}^{\circ o m_{\mathrm{D}-\bmod \left(y_{1}\right)}}\left(\mathcal{F}^{\prime}, \mathcal{F}_{1}\right) \simeq \mathcal{H}^{\circ} m_{\mathrm{D}-\bmod \left(y_{2}\right)}\left(\mathcal{F}, f_{*}\left(\mathcal{F}_{1}\right)\right) .
$$

We have:

$$
\mathcal{H}_{\text {oom }}{\operatorname{D-mod}\left(y_{1}\right)}\left(\mathcal{F}^{\prime}, \mathcal{F}_{1}\right) \simeq \operatorname{Tot}\left(\mathcal{H}_{o m} \operatorname{D-mod}(z \bullet)\left(\left(g^{\bullet}\right)^{*} \circ f^{*}(\mathcal{F}),\left(g^{\bullet}\right)^{*}\left(\mathcal{F}_{1}\right)\right)\right),
$$

which can be rewritten by adjunction as

$$
\begin{aligned}
& \operatorname{Tot}\left(\mathcal{H}_{0} m_{\mathrm{D}-\bmod \left(y_{2}\right)}\left(\mathcal{F},\left(f \circ g^{\bullet}\right)_{*} \circ\left(g^{\bullet}\right)^{*}\left(\mathcal{F}_{1}\right)\right)\right) \simeq \\
& \simeq \mathcal{H}_{0} m_{\mathrm{D}-\bmod \left(y_{2}\right)}\left(\mathcal{F}, \operatorname{Tot}\left(\left(f \circ g^{\bullet}\right)_{*} \circ\left(g^{\bullet}\right)^{*}\left(\mathcal{F}_{1}\right)\right)\right) \text {. }
\end{aligned}
$$

Hence, it suffices to show that the canonical map

$$
f_{*}\left(\mathcal{F}_{1}\right) \rightarrow \operatorname{Tot}\left(\left(f \circ g^{\bullet}\right)_{*} \circ\left(g^{\bullet}\right)^{*}\left(\mathcal{F}_{1}\right)\right)
$$

is an isomorphism. However, this is given by DrGa1, Lemma 7.5.2].

A.2. Step 2. In this subsection we will how that the functors

$$
\left(\iota^{-}\right)^{!} \circ\left(\mathrm{p}^{-}\right)^{*} \text { and }\left(\iota^{+}\right)^{!} \circ\left(\mathrm{p}^{+}\right)^{*}
$$

take values in $\mathrm{D}-\bmod \left(y^{0}\right) \subset \operatorname{Pro}\left(\mathrm{D}-\bmod \left(y^{0}\right)\right)$. Again, by symmetry, it is sufficient to do this for $\left(\iota^{-}\right) ! \circ\left(\mathrm{p}^{-}\right)^{*}$. 
A.2.1. Note that the functor $\left(\iota^{-}\right) !$admits a (possibly non-continuous) right adjoint, denoted $\left(\left(\iota^{-}\right)^{!}\right)^{R}$. Hence, our assertion is that the functor $\left(\mathrm{p}_{-}\right)_{*} \circ\left(\left(\iota^{-}\right)^{!}\right)^{R}$ admits a left adjoint, which takes values in $D-\bmod \left(y^{0}\right)$, rather than in $\operatorname{Pro}\left(D-\bmod \left(y^{0}\right)\right)$.

Since $y$ was assumed QCA, the category $D-\bmod (y)$ is compactly generated. Hence, it is enough to show that the value of $\left(\iota^{-}\right)^{!} \circ\left(\mathrm{p}^{-}\right)^{*}$ on $\mathcal{F} \in \mathrm{D}-\bmod (y)^{c}$ belongs to $\mathrm{D}-\bmod \left(y^{0}\right)$.

A.2.2. Recall from [GR, Sect. 4.2.4] that the category $\operatorname{Pro}\left(\mathrm{D}-\bmod \left(y^{0}\right)\right)$ inherits a t-structure from $D-\bmod \left(y^{0}\right)$. We claim:

Lemma A.2.3. For $\mathcal{F} \in \mathrm{D}-\bmod (\mathrm{y})^{c}$, the object $\left(\iota^{-}\right)^{!} \circ\left(\mathrm{p}^{-}\right)^{*}(\mathcal{F}) \in \operatorname{Pro}\left(\mathrm{D}-\bmod \left(\mathrm{y}^{0}\right)\right)$ is cohomologically bounded, i.e., belongs to $\operatorname{Pro}\left(\mathrm{D}-\bmod \left(\mathrm{y}^{0}\right)\right)^{\geq-n, \leq n}$ for some $n$.

Proof. The fact that $\left(\iota^{-}\right) ! \circ\left(\mathrm{p}^{-}\right)^{*}(\mathcal{F})$ is bounded above follows by combining the following three facts: (i) $\mathcal{F}$ is bounded above; (ii) the functor $\left(\iota^{-}\right)^{!}$has a bounded cohomological amplitude; (iii) the functor $\mathrm{p}_{*}^{-}$, right adjoint to $\left(\mathrm{p}^{-}\right)^{*}$, is left t-exact.

Let us show that $\left(\iota^{-}\right)^{!} \circ\left(\mathrm{p}^{-}\right)^{*}(\mathcal{F})$ is bounded below. Consider the object $\mathbb{D} y(\mathcal{F}) \in \mathrm{D}-\bmod (y)^{c}$, where $\mathbb{D} y$ denotes the Verdier duality functor on $y$ (see [DrGa1, Sect. 7.3.4 and Corollary 8.4.2]).

The functor $\left(\mathrm{p}^{-}\right)^{!}$has a bounded cohomological amplitude, hence $\left(\mathrm{p}^{-}\right)^{!} \circ \mathbb{D} y(\mathcal{F})$ belongs to $\mathrm{D}-\bmod \left(y^{-}\right)^{\leq m}$ for some $m$. Hence, we can write $\left(\mathrm{p}^{-}\right)^{!} \circ \mathbb{D} y(\mathcal{F}) \in \mathrm{D}-\bmod \left(y^{-}\right)$as a filtered colimit

$$
\underset{i}{\lim } \mathcal{F}_{i}^{-},
$$

where $\mathcal{F}_{i}^{-} \in \mathrm{D}-\bmod \left(\mathrm{y}^{-}\right) \leq m$. In this case

$$
\left(\mathrm{p}^{-}\right)^{*}(\mathcal{F}) \simeq \underset{i}{ } \underset{i}{\lim } " \mathbb{D}_{y-}\left(\mathcal{F}_{i}^{-}\right)
$$

However, the functor $\mathbb{D}_{y}$ - has a bounded cohomological amplitude, so all $\mathbb{D}_{y-}\left(\mathcal{F}_{i}^{-}\right)$belong to $\mathrm{D}-\bmod \left(y^{-}\right)^{\geq-l}$ for some $l$. Now,

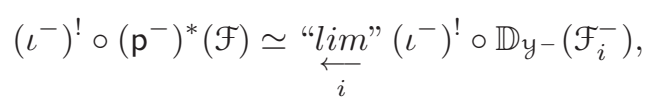

and the boundedness below follows from the fact that the functor $\left(\iota^{-}\right) !$has a bounded cohomological amplitude.

A.2.4. Let us now observe that for any $\mathcal{F} \in \mathrm{D}-\bmod (\mathrm{y})$, the object $\left(\psi^{0}\right)^{!} \circ\left(\iota^{-}\right)^{!} \circ\left(\mathrm{p}^{-}\right)^{*}(\mathcal{F})$ belongs to $\mathrm{D}-\bmod \left(Z^{0}\right)$. Indeed, we rewrite

$$
\left(\psi^{0}\right)^{!} \circ\left(\iota^{-}\right)^{!} \circ\left(\mathrm{p}^{-}\right)^{*} \simeq\left(\iota^{0}\right)^{!} \circ\left(\psi^{-}\right)^{!} \circ\left(\mathrm{p}^{-}\right)^{*},
$$

and the latter is isomorphic, up to a cohomological shift to

$$
\left(i^{-}\right)^{!} \circ\left(\psi^{-}\right)^{*} \circ\left(\mathrm{p}^{-}\right)^{*} \simeq\left(i^{-}\right)^{!} \circ\left(p^{-}\right)^{*} \circ \psi^{*} .
$$

However, $\psi^{*}(\mathcal{F}) \in \mathrm{D}-\bmod (Z)^{\mathbb{G}_{m}-\operatorname{mon}}$, and hence

$$
\left(i^{-}\right)^{!} \circ\left(p^{-}\right)^{*} \circ \psi^{*}(\mathcal{F}) \in \mathrm{D}-\bmod \left(Z^{0}\right)
$$

by Theorem 4.2 .5 .

Since $\psi^{0}$ is smooth (and so $\left(\psi^{0}\right)^{!}$is isomorphic to $\left(\psi^{0}\right)^{*}$ up to a cohomological shift), we obtain that the object $\left(\psi^{0}\right)^{*} \circ\left(\iota^{-}\right)^{!} \circ\left(\mathrm{p}^{-}\right)^{*}(\mathcal{F})$ belongs to $\mathrm{D}-\bmod \left(Z^{0}\right)$ 
A.2.5. Now, the fact that $\left(\iota^{-}\right)^{!} \circ\left(\mathrm{p}^{-}\right)^{*}(\mathcal{F})$ belongs to $\mathrm{D}-\bmod \left(y^{0}\right)$ for $\mathcal{F} \in \mathrm{D}-\bmod (y)^{c}$ follows from Lemma A.2.3 and the next observation:

Lemma A.2.6. Let $g: \mathcal{Z} \rightarrow \mathcal{W}$ be a smooth surjective map between quasi-compact stacks. Let $\widetilde{\mathcal{F}}$ be a cohomologically bounded object of $\operatorname{Pro}(\mathrm{D}-\bmod (\mathcal{W}))$. Then $\widetilde{\mathcal{F}} \in \mathrm{D}-\bmod (\mathcal{W})$ if and only if $g^{*}(\widetilde{\mathcal{F}})$ belongs to $\mathrm{D}-\bmod (Z) \subset \operatorname{Pro}(\mathrm{D}-\bmod (Z))$.

Proof. The "only if" direction is obvious. For the "if" direction we proceed as follows.

Let $g^{\bullet}: z^{\bullet} \rightarrow \mathcal{W}$ be the Čech nerve of $g: z \rightarrow \mathcal{W}$. By assumption, $\left(g^{\bullet}\right)^{*}(\widetilde{\mathcal{F}})$ gives rise to a well-defined object of

$$
\operatorname{Tot}\left(\mathrm{D}-\bmod \left(z^{\bullet}\right)\right)
$$

Applying the inverse of the equivaence

$$
\left(g^{\bullet}\right)^{*}: \mathrm{D}-\bmod (\mathrm{y}) \rightarrow \operatorname{Tot}\left(\mathrm{D}-\bmod \left(z^{\bullet}\right)\right),
$$

from $\left(g^{\bullet}\right)^{*}(\widetilde{\mathcal{F}})$ we obtain a well-defined object of $\mathrm{D}-\bmod (\mathcal{W})$ that we denote by $\mathcal{F}$. We claim that $\widetilde{\mathcal{F}} \simeq \mathcal{F}$.

By construction, the object $\mathcal{F} \in \mathrm{D}-\bmod (y)$ is cohomologically bounded. Assume that $\widetilde{\mathcal{F}}$ belongs to $\operatorname{Pro}(\mathrm{D}-\bmod (\mathcal{W}))^{\geq-n, \leq n}$. By [GR, Sect. 4.2.4], we have a canonical equivalence

$$
\operatorname{Pro}(\mathrm{D}-\bmod (\mathcal{W}))^{\geq-n, \leq n} \simeq \operatorname{Pro}\left(\operatorname{D}-\bmod (\mathcal{W})^{\geq-n, \leq n}\right) .
$$

Hence, in order to establish an isomorphism $\mathcal{F} \simeq \widetilde{\mathcal{F}}$, it is sufficient to construct a functorial isomorphism

$$
\mathcal{H} \operatorname{om}\left(\widetilde{\mathcal{F}}, \mathcal{F}^{\prime}\right) \simeq \mathcal{H} \operatorname{om}\left(\mathcal{F}, \mathcal{F}^{\prime}\right)
$$

for $\mathcal{F}^{\prime} \in \mathrm{D}-\bmod (\mathcal{W})$ which is also cohomologically bounded.

By construction,

$$
\mathcal{H} \operatorname{om}\left(\widetilde{\mathcal{F}}, \mathcal{F}^{\prime}\right) \simeq \operatorname{Tot}\left(\mathcal{H} \text { om }\left(\left(g^{\bullet}\right)^{*}(\widetilde{\mathcal{F}}),\left(g^{\bullet}\right)^{*}\left(\mathcal{F}^{\prime}\right)\right)\right) .
$$

Now, the fact that $\widetilde{\mathcal{F}}$ is bounded above and that $\mathcal{F}^{\prime}$ is bounded below implies that

$$
\operatorname{Tot}\left(\mathcal{H} o m\left(\left(g^{\bullet}\right)^{*}(\widetilde{\mathcal{F}}),\left(g^{\bullet}\right)^{*}\left(\mathcal{F}^{\prime}\right)\right)\right) \simeq \mathcal{H} \operatorname{com}\left(\widetilde{\mathcal{F}}, \operatorname{Tot}\left(\left(g^{\bullet}\right)_{*} \circ\left(g^{\bullet}\right)^{*}\left(\mathcal{F}^{\prime}\right)\right)\right) .
$$

Now,

$$
\mathcal{F}^{\prime} \rightarrow \operatorname{Tot}\left(\left(g^{\bullet}\right)_{*} \circ\left(g^{\bullet}\right)^{*}\left(\mathcal{F}^{\prime}\right)\right)
$$

is an isomorphism since (A.4) is an equivalence.

A.3. Step 3. Having established that both sides of (4.6) belong to D-mod( $\left(y^{0}\right)$, we are finally ready to show that the map (4.6), is an isomorphism.

Since the functor

$$
\left(\psi^{0}\right)^{!}: \mathrm{D}-\bmod \left(\mathrm{y}^{0}\right) \rightarrow \mathrm{D}-\bmod \left(Z^{0}\right)
$$

is conservative, it suffices to show that the natural transformation

$$
\left(\psi^{0}\right)^{*} \circ\left(\iota^{-}\right)^{*} \circ\left(\mathrm{p}^{-}\right)^{*} \rightarrow\left(\psi^{0}\right)^{*} \circ\left(\iota^{+}\right)^{!} \circ\left(\mathrm{p}^{+}\right)^{*},
$$

induced by (4.6), is an isomorphism. 
A.3.1. Consider the map $\beta^{-}: Z^{-} \rightarrow y^{-} \underset{y}{x} Z$. Denote

$$
\mathcal{K}^{-}:=\left(\beta^{-}\right)^{*}\left(\omega_{y-\underset{y}{x} Z}\right) \in \mathrm{D}-\bmod \left(Z^{-}\right) .
$$

The object $\mathcal{K}^{-}$is isomorphic to $\omega_{Z^{-}}$up to a cohomological shift, since both $Z^{-}$and $y^{-} \underset{y}{\times} Z$ are smooth over $y^{-}$.

Note that for $\mathcal{F} \in \mathrm{D}-\bmod (y)$ there exists a canonical isomorphism

$$
\left(\psi^{-}\right)^{*} \circ\left(\mathrm{p}^{-}\right)^{!}(\mathcal{F}) \simeq \mathcal{K}^{-} \stackrel{!}{\otimes}\left(p^{-}\right)^{!} \circ \psi^{*}(\mathcal{F}) .
$$

A.3.2. Consider now the map $\beta^{0}: Z^{0} \rightarrow \underset{y^{+}}{y^{0}} \underset{Z^{+}}{ }$. Denote

$$
\mathcal{K}^{0}:=\left(\beta^{+}\right)^{*}\left(\omega_{y 0} \times Z^{+}\right) \in \mathrm{D}-\bmod \left(Z^{0}\right) .
$$

The object $\mathcal{K}^{0}$ is isomorphic to $\omega_{Z^{0}}$ up to a cohomological shift, since both $Z^{-}$and $y^{0} \underset{y+}{\times} Z^{+}$ are smooth over $y^{0}$.

Note that for $\mathcal{F}^{+} \in \mathrm{D}-\bmod \left(y^{+}\right)$there exists a canonical isomorphism

$$
\left(\psi^{0}\right)^{*} \circ\left(\iota^{+}\right)^{!}\left(\mathcal{F}^{+}\right) \simeq \mathcal{K}^{0} \stackrel{!}{\otimes}\left(i^{+}\right)^{!} \circ\left(\psi^{+}\right)^{*}\left(\mathcal{F}^{+}\right) .
$$

A.3.3. Note now that in the diagram

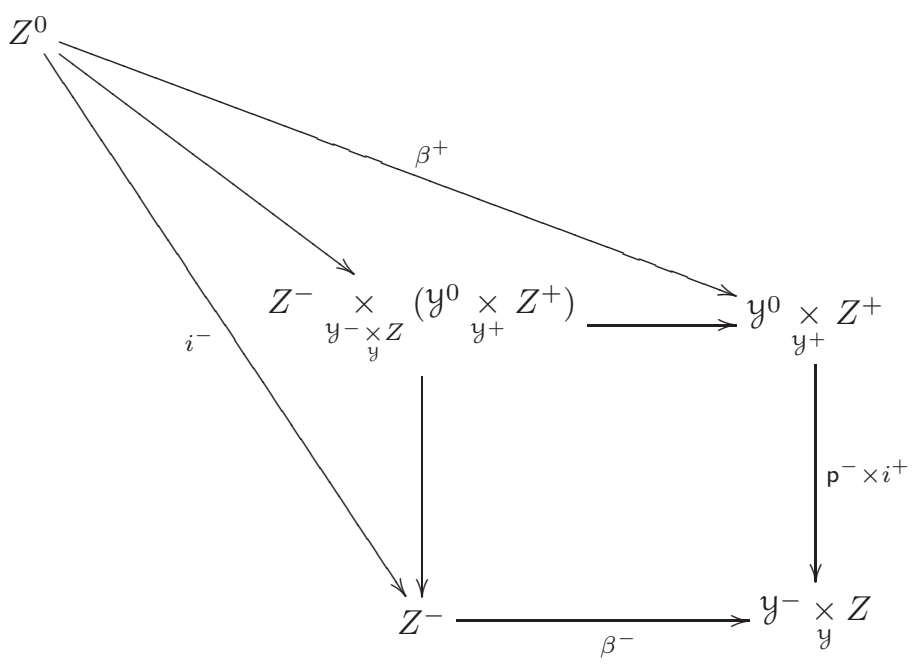

the map

$$
Z^{0} \rightarrow\left(y^{0} \underset{y^{+}}{\times} Z^{+}\right) \underset{y-\underset{y}{\times} Z^{-}}{\times} Z^{-}
$$

is an open embedding. Hence, we obtain a canonical isomorphism

$$
\mathcal{K}^{0} \rightarrow\left(i^{-}\right)^{!}\left(\mathcal{K}^{-}\right) .
$$


A.3.4. Now, diagram chase shows that for $\mathcal{F} \in \mathrm{D}-\bmod (y)$ the following diagram commutes

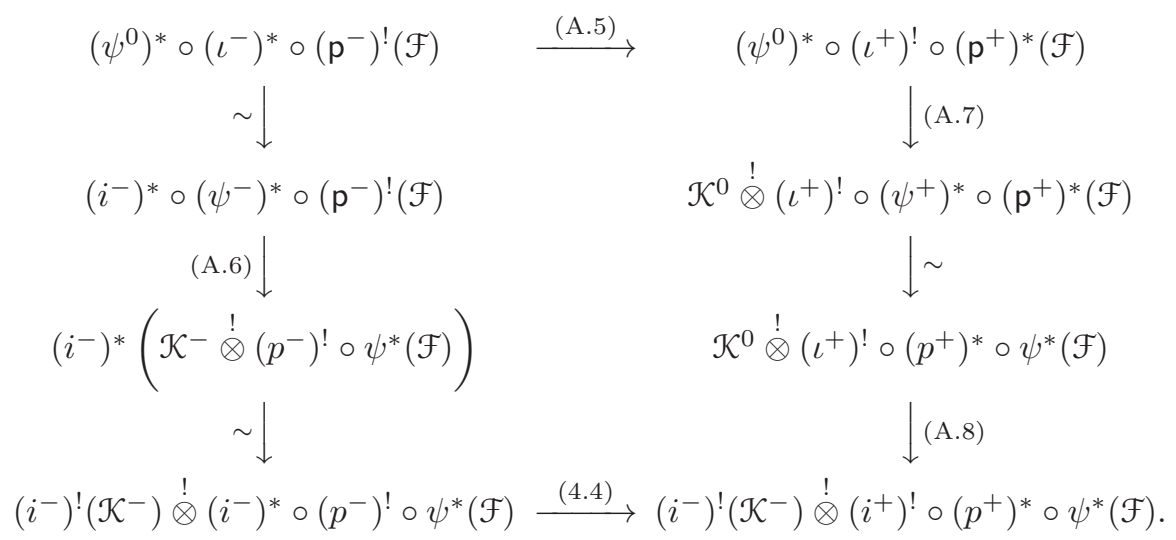

Hence, we obtain that (A.5) is an isomorphism, as desired.

Appendix B. The support of Cuspidal objects of D-mod $\left(\operatorname{Bun}_{G}\right)$

The goal of this Appendix is to prove Proposition 1.4.6, i.e., to construct an open substack $\mathcal{U} \subset \operatorname{Bun}_{G}$ having quasi-compact intersection with each connected component of $\operatorname{Bun}_{G}$ and such that the $*$-support and !-support of each object of $\mathrm{D}$-mod $\left(\operatorname{Bun}_{G}\right)_{\text {cusp }}$ are contained in $\mathcal{U}$.

\section{B.1. Definition of $\mathcal{U}$.}

B.1.1. Let $\Lambda_{G}$ denote the coweight lattice of $G$. Set $\Lambda_{G}^{\mathbb{Q}}:=\Lambda_{G} \otimes \mathbb{Q}$ and let $\Lambda_{G}^{+, \mathbb{Q}} \subset \Lambda_{G}^{\mathbb{Q}}$ be the dominant cone.

We will use the Harder-Narasimhan-Shatz stratification of Bun ${ }_{G}$ (see DrGa2, Sect. 7.4]).

Its strata are labeled by elements $\lambda \in \Lambda_{G}^{+, \mathbb{Q}}$ and denoted by $\operatorname{Bun}_{G}^{(\lambda)}$. The set of those $\lambda$ for which $\operatorname{Bun}_{G}^{(\lambda)} \neq \emptyset$ is discrete in $\Lambda_{G} \otimes \mathbb{R}$.

B.1.2. Set

$$
\mathcal{U}:=\bigcup_{\lambda \in \Sigma} \operatorname{Bun}_{G}^{(\lambda)},
$$

where $\Sigma$ is the set of all $\lambda \in \Lambda_{G}^{+, \mathbb{Q}}$ such that the image of $\lambda$ in $\Lambda_{G_{\text {adj }}}^{\mathbb{Q}}$ is $\leq(2 g-2) \rho_{G}$. Here $g$ is the genus of the curve $X, \rho_{G} \in \Lambda_{G_{\text {adj }}}^{\mathbb{Q}}$ is the half-sum of positive coroots, and the ordering $\leq$ is the usual one (i.e., $\lambda_{1} \leq \lambda_{2}$ if $\lambda_{2}-\lambda_{1}$ is a linear combination of simple coroots with non-negative coefficients).

Lemma B.1.3. The union of strata $U$ is an open substack of $\mathrm{Bun}_{G}$. Its intersection with each connected component of $\mathrm{Bun}_{G}$ is quasi-compact.

Proof. Use the standard properties of the Harder-Narasimhan-Shatz stratification (see DrGa2, Lemma 7.4.9]) and the following obvious fact: for any $\lambda_{0} \in \Lambda_{G}^{\mathbb{Q}}$, the union of $\operatorname{Bun}_{G}^{(\lambda)}$ for $\lambda \in\left(\lambda_{0}+\Lambda_{[G, G]}^{\mathbb{Q}}\right) \cap \Lambda_{G}^{+, \mathbb{Q}}$ is both open and closed.

\section{B.2. Statement of the result.}


B.2.1. Let $\jmath: \mathfrak{U} \hookrightarrow \operatorname{Bun}_{G}$ denote the embedding. In Sect. B.4 we will prove the following

Proposition B.2.2. For any $\mathcal{F} \in \mathrm{D}-\bmod \left(\operatorname{Bun}_{G}\right)_{\text {cusp }}$, the canonical maps

$$
\jmath ! \circ \jmath^{*}(\mathcal{F}) \rightarrow \mathcal{F} \rightarrow \jmath_{*} \circ \jmath^{*}(\mathcal{F})
$$

are isomorphisms.

The statement about $\jmath ! \circ \jmath^{*}(\mathcal{F})$ will be understood as follows: the partially defined 12 functor $\jmath$ ! is defined on $\jmath^{*}(\mathcal{F})$ and the map $\jmath ! \circ \jmath^{*}(\mathcal{F}) \rightarrow \mathcal{F}$ is an isomorphism.

Remark B.2.3. If $G$ is a torus then $\Sigma=\Lambda_{G}^{+, \mathbb{Q}}=\Lambda_{G}^{\mathbb{Q}}, \mathcal{U}=\operatorname{Bun}_{G}$, and D-mod $\left(\operatorname{Bun}_{G}\right)_{\text {cusp }}=$ $\mathrm{D}-\bmod \left(\operatorname{Bun}_{G}\right)$. So the proposition holds tautologically.

Remark B.2.4. If $g=0$ and $G$ is not a torus then $\Sigma=\emptyset$ and therefore $\mathcal{U}=\emptyset$. In this case the proposition says that $\mathrm{D}-\bmod \left(\operatorname{Bun}_{G}\right)_{\text {cusp }}=0$.

Remark B.2.5. Let $g=0$ and $\mathcal{F} \in \mathrm{D}-\bmod \left(\operatorname{Bun}_{G}\right)$. If the map $\mathcal{F} \rightarrow \jmath_{*} \circ \jmath^{*}(\mathcal{F})$ is an isomorphism then so is the map $\jmath ! \circ \jmath^{*}(\mathcal{F}) \rightarrow \mathcal{F}$. This follows from the fact that the open substack $\mathcal{U} \subset \operatorname{Bun}_{G}$ equals either $\emptyset$ or $\operatorname{Bun}_{G}$ (see Remarks B.2.3 B.2.4).

\section{B.3. The key lemma.}

B.3.1. Recall that for every parabolic $P \subset G$ we denote by

$$
\mathrm{p}: \mathrm{Bun}_{P} \rightarrow \operatorname{Bun}_{G} \text { and } \mathrm{q}: \mathrm{Bun}_{P} \rightarrow \operatorname{Bun}_{M}
$$

the corresponding morphisms (as usual, $M$ is the Levi quotient of $P$ ).

Lemma B.3.2. Let $\Sigma$ be as in Sect. B.1. Let $\lambda \in \Lambda_{G}^{+, \mathbb{Q}}, \lambda \notin \Sigma$. Then there exists a proper parabolic $P \subset G$ and a locally closed substack $V \subset \operatorname{Bun}_{M}$ such that:

(1) The fiber of $\mathrm{q}: \operatorname{Bun}_{P} \rightarrow \operatorname{Bun}_{M}$ over any geometric point of $V$ has only one isomorphism class of geometric points;

(2) $\mathrm{q}^{-1}(V) \subset \mathrm{p}^{-1}\left(\operatorname{Bun}_{G}^{(\lambda)}\right)$;

(3) The morphism $\mathrm{p}_{\mathrm{q}^{-1}(V)}: \mathrm{q}^{-1}(V) \rightarrow \mathrm{Bun}_{G}^{(\lambda)}$ is an isomorphism if $g>0$ and is surjective if $g=0$.

Remark B.3.3. Let $N(P)$ denote the unipotent radical of $P$. For any $\mathcal{P} \in \operatorname{Bun}_{M}$ let $N(P)_{\mathcal{P}}$ denote the group-scheme $N(P)_{\mathcal{P}}$ over $X$ (here we regard $M$ as acting on $N(P)$ via the embedding $M \hookrightarrow P)$. Then the fiber of q over $\mathcal{P} \in \operatorname{Bun}_{M}$ is the stack of $N(P)_{\mathcal{P}}$-torsors on $X$. Condition (1) in Lemma B.3.2 says that if $\mathcal{P} \in V$ then all such torsors are trivial.

Proof. Let us first assume that $g=0$, i.e., $X=\mathbb{P}^{1}$. We can also assume that $G$ is not a torus (otherwise $\Sigma=\Lambda_{G}^{+, \mathbb{Q}}$ and there is nothing to prove). Take $P=B$ to be a Borel subgroup (so $M=T$ is the Cartan). Define $V \subset \operatorname{Bun}_{T}$ to be the substack of $T$-bundles of degree $\lambda$. Property (1) follows from the fact that $H^{1}\left(\mathbb{P}^{1}, \mathcal{O}(n)\right)=0$ for $n \geq 0$. Property (3) holds because any $G$ bundle on $\mathbb{P}^{1}$ admits a reduction to a maximal torus of $G$ (see [BN] and references therein). Property (2) is clear.

Let us now assume that $X$ has genus $g>0$. The simple roots of $G$ will be denoted by $\check{\alpha}_{i}$. Define $P$ and $M$ by the following condition: the simple roots of $M$ are those $\check{\alpha}_{i}$ 's for which $\left\langle\lambda, \check{\alpha}_{i}\right\rangle \leq 2 g-2$. We have $P \neq G$ (indeed, let $\bar{\lambda} \in \Lambda_{G_{\text {adj }}}^{+, \mathbb{Q}}$ be the image of $\lambda$, then the equality $P=G$ would imply that $(2 g-2) \rho_{G}-\bar{\lambda}$ is dominant and therefore $\bar{\lambda} \leq(2 g-2) \rho_{G}$, contrary to the assumption that $\lambda \notin \Sigma)$.

\footnotetext{
${ }^{12}$ In fact, the embedding $\jmath: U \hookrightarrow \operatorname{Bun}_{G}$ has the remarkable property that the functor $\jmath !$ is defined on all of D-mod(U), see DrGa2 Theorem 9.1.2]. We will not use this fact.
} 
Note that $\Lambda_{G}^{+, \mathbb{Q}} \subset \Lambda_{M}^{+, \mathbb{Q}}$, so we can consider the Harder-Narasimhan-Shatz stratum $\operatorname{Bun}_{M}^{(\lambda)}$. Set $V:=\operatorname{Bun}_{M}^{(\lambda)}$. Then $V$ has the required properties. Properties (2)-(3) follow from the definition of the Harder-Narasimhan-Shatz strata (see [DrGa2, Theorem 7.4.3]) and the assumption $2 g-2 \geq 0$. Remark B.3.3 shows that to prove property (1) it suffices to check the equality

$$
H^{1}\left(X, \mathfrak{n}_{\mathcal{P}}\right)=0 \text { for all } \mathcal{P} \in \operatorname{Bun}_{M}^{(\lambda)},
$$

where $\mathfrak{n}:=\operatorname{Lie}(N)$. This equality holds by DrGa2, Proposition 10.1.3] and the characteristic 0 assumption on $k$.

B.3.4. In the situation of Lemma B.3.2 consider the corresponding morphism

$$
\mathrm{q}: \mathrm{q}^{-1}(V) \rightarrow V
$$

\section{Lemma B.3.5.}

(i) Each fiber of the morphism $\mathbf{q}: \mathrm{q}^{-1}(V) \rightarrow V$ is a classifying space of a unipotent group.

(ii) The functors

$$
\mathrm{q}_{*}: \mathrm{D}-\bmod \left(\mathrm{q}^{-1}(V)\right) \rightarrow \mathrm{D}-\bmod (V) \text { and } \mathrm{q} !: \mathrm{D}-\bmod \left(\mathrm{q}^{-1}(V)\right) \rightarrow \mathrm{D}-\bmod (V)
$$

are equivalences (that differ by a cohomological shift), with the inverses provided by

$$
\mathrm{q}^{*}: \mathrm{D}-\bmod (V) \rightarrow \mathrm{D}-\bmod \left(\mathrm{q}^{-1}(V)\right) \text { and } \mathrm{q}^{!}: \mathrm{D}-\bmod (V) \rightarrow \mathrm{D}-\bmod \left(\mathrm{q}^{-1}(V)\right),
$$

respectively.

Proof. Statement (i) follows from the interpretation of the fibers of q given in Remark B.3.3. Statement (ii) follows from (i) because q is smooth.

\section{B.4. Proof of Proposition B.2.2.}

B.4.1. Let $\Sigma$ be as in Sect. B.1 Let $\lambda \in \Lambda_{G}^{+, \mathbb{Q}}, \lambda \notin \Sigma$. Let $\imath_{\lambda}: \operatorname{Bun}_{G}^{(\lambda)} \hookrightarrow \operatorname{Bun}_{G}$ be the corresponding locally closed embedding. We need to show that for any cuspidal $\mathcal{F} \in$ $\mathrm{D}-\bmod \left(\operatorname{Bun}_{G}\right)$ one has

$$
i_{\lambda}^{!}(\mathcal{F})=0, \quad \imath_{\lambda}^{*}(\mathcal{F})=0 .
$$

Let $P$ and $V$ be as in Lemma B.3.2. Consider the corresponding morphisms

$$
\left.\mathrm{p}\right|_{\mathbf{q}^{-1}(V)}: \mathrm{q}^{-1}(V) \rightarrow \mathrm{Bun}_{G}^{(\lambda)} \text { and } \mathbf{q}_{\mathbf{q}^{-1}(V)}: \mathrm{q}^{-1}(V) \rightarrow V .
$$

B.4.2. Let us first prove that $\imath_{\lambda}^{!}(\mathcal{F})=0$. By cuspidality, $\mathbf{q}_{*}\left(\mathrm{p}^{!}(\mathcal{F})\right)=0$, so

$$
\left(\left.\mathrm{q}\right|_{\mathbf{q}^{-1}(V)}\right)_{*} \circ\left(\left.\mathrm{p}\right|_{\mathbf{q}^{-1}(V)}\right)^{!} \circ \imath_{\lambda}^{!}(\mathcal{F})=0 .
$$

By Lemma B.3.5(ii), this means that $\left(\left.\mathrm{p}\right|_{\mathrm{q}^{-1}(V)}\right) ! \circ !(\mathcal{F})=0$. Since $\left.\mathrm{p}\right|_{\mathrm{q}^{-1}(V)}: \mathrm{q}^{-1}(V) \rightarrow \operatorname{Bun}_{G}^{(\lambda)}$ is surjective, the functor $\left(\left.\mathrm{p}\right|_{\mathrm{q}^{-1}(V)}\right) !$ is conservative by [DrGa1, Lemma 5.1.6]. So $\imath_{\lambda}^{!}(\mathcal{F})=0$. 
B.4.3. Let us now prove that $\imath_{\lambda}^{*}(\mathcal{F})=0$. By Remark B.2.5. we can assume that $g>0$, so the map $\left.\right|_{\mathrm{q}^{-1}(V)}: \mathrm{q}^{-1}(V) \rightarrow \operatorname{Bun}_{G}^{(\lambda)}$ is an isomorphism (see Lemma B.3.2).

We have $\mathrm{CT}_{!}^{\lambda}(\mathcal{F})=0$, where $\mathrm{CT}_{!}^{\lambda}$ is the constant term functor with respect to $P$. So the adjunction from Theorem 1.2 .3 implies that $\operatorname{Hom}\left(\mathcal{F}, \operatorname{Eis}_{*}^{\lambda}(\mathcal{E})\right)=0$ for all $\mathcal{E} \in \operatorname{D-mod}\left(\operatorname{Bun}_{M}^{\lambda}\right)$.

Taking $\mathcal{E}$ to be the $*$-pushforward of an object $\mathcal{E}^{\prime} \in \mathrm{D}$-mod$(V)$ we have

$$
\operatorname{Eis}_{*}(\mathcal{E}) \simeq\left(\imath_{\lambda}\right)_{*} \circ\left(\left.\mathbf{p}\right|_{\mathbf{q}^{-1}(V)}\right)_{*} \circ\left(\left.\mathbf{q}\right|_{\mathbf{q}^{-1}(V)}\right)^{!}\left(\mathcal{E}^{\prime}\right)
$$

and hence

$$
\operatorname{Hom}\left(\mathcal{F},\left(\imath_{\lambda}\right)_{*} \circ\left(\left.\mathbf{p}\right|_{\mathbf{q}^{-1}(V)}\right)_{*} \circ\left(\left.\mathbf{q}\right|_{\mathbf{q}^{-1}(V)}\right)^{!}\left(\mathcal{E}^{\prime}\right)\right)=0 .
$$

Since $\left(\left.p\right|_{\mathbf{q}^{-1}(V)}\right)_{*}$ and $\left(\left.\mathbf{q}\right|_{\mathbf{q}^{-1}(V)}\right) !$ are equivalences, we obtain

$$
\operatorname{Hom}\left(\mathcal{F},\left(\imath_{\lambda}\right)_{*}\left(\mathcal{F}^{\prime}\right)\right)=0, \quad \forall \mathcal{F}^{\prime} \in \mathrm{D}-\bmod \left(\operatorname{Bun}_{G}^{(\lambda)}\right),
$$

i.e., $\imath_{\lambda}^{*}(\mathcal{F})=0$.

\section{Appendix C. Proof of Proposition 2.4.4}

In this Appendix (just as in Proposition 2.4.4 itself) the characteristic of the ground field $k$ can be arbitrary.

\section{C.1. Reduction of Proposition 2.4.4 to Lemma C.1.3.}

C.1.1. We have to prove that the quotient $\left(\mathbb{A}^{1} \times G \times G\right) / \widetilde{G}$ is a quasi-affine scheme. We know that this quotient exists as an algebraic space (because $\widetilde{G}$ is flat over $\mathbb{A}^{1}$ ). This space is separated because $\widetilde{G}$ is closed in $\mathbb{A}^{1} \times G \times G$.

C.1.2. Recall that a separated quasi-finite morphism is quasi-affine (see [LM, Theorem A.2] or [ Kn, Ch. II, Theorem 6.15]). So it remains to construct a quasi-finite morphism from $\left(\mathbb{A}^{1} \times G \times G\right) / \widetilde{G}$ to an affine scheme $Y$.

Thus it remains to prove the following lemma:

Lemma C.1.3. There exists an affine scheme $Y$ equipped with an action of $G \times G$ and a morphism $f: \mathbb{A}^{1} \rightarrow Y$ such that for any $t \in \mathbb{A}^{1}(k)$ the stabilizer of $f(t)$ contains $\widetilde{G}_{t}$ as a subgroup of finite index.

\section{C.2. Plan of the proof of Lemma C.1.3.}

C.2.1. For each dominant weight $\check{\lambda}$ of $G$ we will construct a finite-dimensional $(G \times G)$-module $R_{\check{\lambda}}$, equipped with a polynomial map $f_{\check{\lambda}}: \mathbb{A}^{1} \rightarrow R_{\check{\lambda}}$.

Let $\operatorname{Stab}_{t}^{\check{\lambda}} \subset G \times G$ denote the stabilizer of $f_{\check{\lambda}}(t)$. We will show that

$$
\left(\bigcap_{\check{\lambda}} \operatorname{Stab}_{t}^{\check{\lambda}}\right)_{\text {red }}=\widetilde{G}_{t} \text {. }
$$


C.2.2. This will immediately imply Lemma C.1.3 indeed, one can take

$$
Y=\prod_{\check{\lambda}} R_{\check{\lambda}}, \quad f=\left(f_{\check{\lambda}}\right),
$$

where $\check{\lambda}$ runs through the set of all dominant weights of $G$ (or if you prefer, a sufficiently large finite collection of weights).

Recall that $\widetilde{G}_{t}$ is reduced by Proposition 2.3.8(i), so (C.1) is equivalent to the inclusions

$$
\left(\bigcap_{\check{\lambda}} \operatorname{Stab}_{t}^{\check{\lambda}}\right)_{\text {red }} \subset \widetilde{G}_{t} \subset \bigcap_{\check{\lambda}} \operatorname{Stab}_{t}^{\check{\lambda}} .
$$

Thus it remains to construct $R_{\check{\lambda}}$ and $f_{\check{\lambda}}$, and to prove (C.2).

\section{C.3. Construction of $R_{\check{\lambda}}$ and $f_{\check{\lambda}}(t)$.}

C.3.1. Definition of $R_{\check{\lambda}}$. Let $V_{\check{\lambda}}$ denote the irreducible $G$-module with highest weight $\check{\lambda}$. Set $R_{\check{\lambda}}:=\operatorname{End}_{k}\left(V_{\check{\lambda}}\right)$. We have a homomorphism of monoids

$$
\rho_{\check{\lambda}}: G \rightarrow \operatorname{End}_{k}\left(V_{\check{\lambda}}\right) .
$$

The action of $G \times G$ on $R_{\check{\lambda}}:=\operatorname{End}_{k}\left(V_{\check{\lambda}}\right)$ is defined as follows: to $\left(g_{1}, g_{2}\right) \in G \times G$ we associate the operator

$$
\operatorname{End}_{k}\left(V_{\check{\lambda}}\right) \rightarrow \operatorname{End}_{k}\left(V_{\check{\lambda}}\right), \quad A \mapsto \rho_{\check{\lambda}}\left(g_{1}\right) \cdot A \cdot \rho_{\check{\lambda}}\left(g_{2}\right)^{-1} .
$$

C.3.2. Definition of $f_{\check{\lambda}}$. Recall that to define $\widetilde{G}$ we fixed in Sect. 2.13 a co-character

$$
\gamma: \mathbb{G}_{m} \rightarrow M
$$

mapping to the center of $M$, which is dominant and regular with respect to $P$. Let us also fix a maximal torus $T \subset G$ contained in $M$ and a Borel $B \subset G$ such that $T \subset B \subset P$.

Now, for $t \in \mathbb{G}_{m}$ we set

$$
f_{\check{\lambda}}(t):=\check{\lambda}(\gamma(t)) \cdot \rho_{\check{\lambda}}\left(\gamma(t)^{-1}\right) .
$$

Thus we get a homomorphism of algebraic groups $\mathbb{G}_{m} \rightarrow \operatorname{Aut}_{k}\left(V_{\check{\lambda}}\right)$. It extends to a homomorphism of algebraic monoids

$$
f_{\check{\lambda}}: \mathbb{A}^{1} \rightarrow \operatorname{End}_{k}\left(V_{\check{\lambda}}\right)
$$

because $\gamma$ is dominant and all the weights of $T$ in $V_{\check{\lambda}}$ are $\leq \check{\lambda}$.

C.3.3. The operator $f_{\check{\lambda}}(0)$. Set $S_{\check{\lambda}}:=f_{\check{\lambda}}(0)$. Then $S_{\check{\lambda}}$ is a $T$-equivariant idempotent operator $V_{\check{\lambda}} \rightarrow V_{\check{\lambda}}$. The subspaces

$$
V_{\check{\lambda}}^{\prime}:=\operatorname{Im}\left(S_{\check{\lambda}}\right), \quad V_{\check{\lambda}}^{\prime \prime}:=\operatorname{Ker}\left(S_{\check{\lambda}}\right)
$$

can be described in terms of the decomposition

$$
V_{\check{\lambda}}=\bigoplus_{\check{\mu}} V_{\check{\lambda}, \check{\mu}}
$$

with respect to the weights of $T$. Namely,

$$
V_{\check{\lambda}}^{\prime}=\bigoplus_{\breve{\mu} \in A} V_{\check{\lambda}, \check{\mu}}, \quad V_{\check{\lambda}}^{\prime \prime}=\bigoplus_{\breve{\mu} \notin A} V_{\check{\lambda}, \check{\mu}}
$$

where $A$ is the set of weights $\check{\mu}$ such that $\check{\lambda}-\check{\mu}$ is in the root lattice of $M$ (which is a sublattice in that of $G$ ).

\section{C.4. The stabilizers $\operatorname{Stab}_{t}^{\check{\lambda}}$.}


C.4.1. Let $\operatorname{Stab}_{t}^{\check{\lambda}} \subset G \times G$ denote the stabilizer of $f_{\check{\lambda}}(t) \in \operatorname{End}_{k}\left(V_{\check{\lambda}}\right)$ with respect to the action of $G \times G$ defined in Sect. C.3.1. Explicitly,

$$
\operatorname{Stab}_{t}^{\check{\lambda}}=\left\{\left(g_{1}, g_{2}\right) \in G \times G \mid \rho_{\check{\lambda}}\left(g_{1}\right) \cdot f_{\check{\lambda}}(t)=f_{\check{\lambda}}(t) \cdot \rho_{\check{\lambda}}\left(g_{2}\right)\right\} .
$$

Our goal is to prove the inclusions (C.2).

C.4.2. The case $t \neq 0$. Let $H_{\check{\lambda}} \subset G$ denote the kernel of $\rho_{\check{\lambda}}: G \rightarrow \operatorname{Aut}_{k}\left(V_{\check{\lambda}}\right)$.

Lemma C.4.3. If $t \neq 0$ then $\operatorname{Stab}_{t}^{\check{\lambda}}=\widetilde{G}_{t} \cdot\left(H_{\check{\lambda}} \times H_{\check{\lambda}}\right)$ and

$$
\bigcap_{\check{\lambda}} \operatorname{Stab}_{t}^{\check{\lambda}}=\widetilde{G}_{t} \cdot\left(\bigcap_{\check{\lambda}}\left(H_{\check{\lambda}} \times H_{\check{\lambda}}\right)\right) .
$$

Proof. Recall that $\widetilde{G}_{t}=\left\{\left(g_{1}, g_{2}\right) \in G \times G \mid g_{2}=\gamma(t) \cdot g_{1} \cdot \gamma(t)^{-1}\right\}$. On the other hand, by (C.3) and (C.4), we have

$$
\operatorname{Stab}_{t}^{\check{\lambda}}=\left\{\left(g_{1}, g_{2}\right) \in G \times G \mid \rho_{\check{\lambda}}\left(g_{2}\right)=\rho_{\check{\lambda}}(\gamma(t)) \cdot \rho_{\check{\lambda}}\left(g_{1}\right) \cdot \rho_{\check{\lambda}}\left(\gamma(t)^{-1}\right)\right\} .
$$

The lemma follows.

The inclusions (C.2) for $t \neq 0$ follow from the above lemma and the next one.

Lemma C.4.4. The group $\left(\bigcap_{\check{\lambda}} H_{\check{\lambda}}\right)_{\text {red }}$ is trivial.

Proof. $\left(\bigcap_{\check{\lambda}} H_{\check{\lambda}}\right)_{\text {red }}$ is a reduced normal subgroup of $G$, which has trivial intersection with $T$. The lemma follows.

Remark C.4.5. If $k$ has characteristic 2 and $G=P G L(2)$ then the group scheme $\bigcap_{\check{\lambda}} H_{\check{\lambda}}$ has order 4. In fact, it equals the kernel of the homomorphism $g: P G L(2) \rightarrow S L(2)$ such that the composition $S L(2) \rightarrow P G L(2) \stackrel{g}{\longrightarrow} S L(2)$ is the Frobenius endomorphism of $S L(2)$.

C.4.6. The case $t=0$. Now let us study $\operatorname{Stab}_{0}^{\check{\lambda}}$ (i.e., the stabilizer $\operatorname{Stab}_{t}^{\check{\lambda}}$ for $t=0$ ). By (C.4), we have

$$
\operatorname{Stab}_{0}^{\check{\lambda}}=\left\{\left(g_{1}, g_{2}\right) \in G \times G \mid \rho_{\check{\lambda}}\left(g_{1}\right) \cdot S_{\check{\lambda}}=S_{\check{\lambda}} \cdot \rho_{\check{\lambda}}\left(g_{2}\right)\right\},
$$

where $S_{\check{\lambda}}:=f_{\check{\lambda}}(0)$.

Note that the subspace $V_{\check{\lambda}}^{\prime} \subset V_{\check{\lambda}}$ is $M$-stable. So we have a homomorphism $M \rightarrow \operatorname{Aut}_{k}\left(V_{\breve{\lambda}}^{\prime}\right)$ and therefore homomorphisms

$$
P \rightarrow M \rightarrow \operatorname{Aut}_{k}\left(V_{\check{\lambda}}^{\prime}\right), \quad P^{-} \rightarrow M \rightarrow \operatorname{Aut}_{k}\left(V_{\check{\lambda}}^{\prime}\right)
$$

Lemma C.4.7. The intersection $\operatorname{Stab}_{0}^{\check{\lambda}} \cap\left(P \times P^{-}\right)$is equal to the fiber product of $P$ and $P^{-}$ over $\operatorname{Aut}_{k}\left(V_{\check{\lambda}}^{\prime}\right)$.

Proof. Follows from (C.5 and the description of $S_{\check{\lambda}}$ given in Sect. C.3.3.

Recall that $\widetilde{G}_{0}=P \underset{M}{\times} P^{-}$. The above lemma implies that

$$
\left(\bigcap_{\check{\lambda}} \operatorname{Stab}_{0}^{\check{\lambda}}\right) \cap\left(P \times P^{-}\right)=P \underset{M / H}{\times} P^{-}
$$


where

$$
H:=\bigcap_{\check{\lambda}} \operatorname{Ker}\left(M \rightarrow \operatorname{Aut}_{k}\left(V_{\grave{\lambda}}^{\prime}\right)\right)
$$

Similarly to Lemma C.4.4 the group $H_{\text {red }}$ is trivial. Therefore, to prove (C.2) for $t=0$ it remains to show that

$$
\left(\bigcap_{\check{\lambda}} \operatorname{Stab}_{0}^{\check{\lambda}}\right)_{\text {red }} \subset P \times P^{-}
$$

This follows from the next lemma.

Lemma C.4.8. If $\check{\lambda}$ is strictly dominant then $\left(\mathrm{Stab}_{0}^{\check{\lambda}}\right)_{\mathrm{red}} \subset P \times P^{-}$.

Proof. Set $V_{\check{\lambda}}^{\prime \prime}:=\operatorname{Ker}\left(S_{\check{\lambda}}\right)$. It is clear that $\operatorname{Stab}_{0}^{\check{\lambda}} \subset K \times K^{-}$, where

$$
K:=\left\{g \in G \mid \rho_{\check{\lambda}}(g)\left(V_{\check{\lambda}}^{\prime}\right)=V_{\check{\lambda}}^{\prime}\right\}, \quad K^{-}:=\left\{g \in G \mid \rho_{\check{\lambda}}(g)\left(V_{\check{\lambda}}^{\prime \prime}\right)=V_{\check{\lambda}}^{\prime \prime}\right\} .
$$

So it remains to show that $K_{\text {red }}=P, K_{\text {red }}^{-}=P^{-}$. Let us prove that $K_{\text {red }}=P$ (the proof of the other equality is similar).

Clearly $P \subset K$, so $K_{\text {red }}$ is a parabolic containing $P$. Thus if $K_{\text {red }} \neq P$ then $K_{\text {red }}$ contains the subgroup $S L(2)$ corresponding to some simple root $\check{\alpha}_{i}$ of $G$ which is not a root of $M$. Then the weight $\check{\lambda}-\left\langle\check{\lambda}, \alpha_{i}\right\rangle \cdot \check{\alpha}_{i}$ should occur in the weight decomposition of $V_{\check{\lambda}}^{\prime}$ with respect to $T$. By the definition of $V_{\grave{\lambda}}^{\prime}$, this means that $\left\langle\check{\lambda}, \alpha_{i}\right\rangle=0$, which is contrary to the assumption that $\check{\lambda}$ is strictly dominant.

Thus we have proved Lemma C.1.3 and Proposition 2.4.4.

Remark C.4.9. It is not hard to show that if char $k \neq 2$ or if the group $[G, G]$ is simply connected then the equality (C.1) can be replaced by the stronger equality

$$
\bigcap_{\check{\lambda}} \operatorname{Stab}_{t}^{\check{\lambda}}=\widetilde{G}_{t}
$$

On the other hand, (C.6) does not hold if $\operatorname{char} k=2$ and $G=P G L(2)$ (this follows from Remark C.4.5.

Remark C.4.10. In Sect. C.3.1 we defined the $(G \times G)$-module $R_{\check{\lambda}}$ to be equal to $\operatorname{End}_{k}\left(V_{\check{\lambda}}\right)$, where $V_{\check{\lambda}}$ is the irreducible $G$-module with highest weight $\check{\lambda}$. The reader may prefer to define $R_{\check{\lambda}}$ by

$$
R_{\check{\lambda}}:=\operatorname{End}_{k}\left(\Delta_{\check{\lambda}}\right) \times \operatorname{End}_{k}\left(\nabla_{\check{\lambda}}\right),
$$

where $\Delta_{\check{\lambda}}\left(\operatorname{resp} . \nabla_{\check{\lambda}}\right)$ is obtained from the 1-dimensional $B$-module corresponding to $\check{\lambda}$ by applying the functor

$$
\{B \text {-modules }\} \rightarrow\{G \text {-modules }\}
$$

left adjoint (resp. right adjoint) to the restriction functor $\{G$-modules $\} \rightarrow\{B$-modules $\}$. It is not hard to show that if one defines $R_{\check{\lambda}}$ by (C.7) then the equality (C.6) holds for any reductive $G$ and any characteristic of $k$. 


\section{Appendix D. Relation to Vinberg's SEMI-Group}

In Sect. D.6 we will explain how the group-scheme $\widetilde{G}$ of Sect. 2.4 can be obtained from the Vinberg semi-group corresponding to $G$ (a.k.a. enveloping semigroup of $G$ ).

Before doing this, we give a brief review of the standard material on the Vinberg semigroup 13 , which is contained in $\mathrm{Vi}, \mathrm{Re}, \mathrm{Ri1}, \mathrm{Ri3}, \mathrm{Bo}$. For the general theory of reductive monoids, see $\mathrm{Pu}, \mathrm{Re}, \mathrm{Vi}$ and $\mathrm{Ti}, \mathrm{Ch} .5$, Sect. 27].

In this Appendix (as in Sect. 2.4) the characteristic of the ground field $k$ can be arbitrary.

\section{D.1. The group $G_{\mathrm{enh}}$.}

D.1.1. Let $G$ be a reductive group. Let $T$ denote its abstract Cartan. Let $\check{\Lambda}_{G}=\check{\Lambda}_{T}$ denote the weight lattice of $G$ (=the lattice of characters of $T$ ). It is equipped with the usual partial order relation, denoted by $\leq$.

D.1.2. Let $Z(G)$ denote the center of $G$. Consider the group

$$
G_{\text {enh }}:=(G \times T) / Z(G),
$$

where $Z(G)$ maps to $G \times T$ anti-diagonally. Note that $Z\left(G_{\text {enh }}\right)=T$.

Set $T_{\text {adj }}:=T / Z(G)$. We have a canonical homomorphism of algebraic groups

$$
G_{\mathrm{enh}}:=(G \times T) / Z(G) \rightarrow T / Z(G)=T_{\text {adj }} .
$$

\section{D.2. Vinberg's semi-group: definition.}

D.2.1. The Vinberg semi-group of $G$, denoted by $\overline{G_{\text {enh }}}$, is an affine algebraic monoid containing $G_{\text {enh }}$ as its group of invertible elements. Such a monoid is uniquely determined by the full monoidal subcategory

$$
\operatorname{Rep}\left(\overline{G_{\text {enh }}}\right) \subset \operatorname{Rep}\left(G_{\text {enh }}\right)
$$

consisting of those representations for which the action of $G_{\text {enh }}$ extends to that of $\overline{G_{\text {enh }}}$. The coordinate ring of $\overline{G_{\mathrm{enh}}}$ is reconstructed from $\operatorname{Rep}\left(\overline{G_{\mathrm{enh}}}\right)$ as the set of matrix elements of all representations from $\operatorname{Rep}\left(\overline{G_{\text {enh }}}\right)$.

The category $\operatorname{Rep}\left(\overline{G_{\text {enh }}}\right)$ is the following. Note that an object $V \in \operatorname{Rep}\left(G_{\text {enh }}\right)$ canonicaly splits as

$$
V \simeq \underset{\check{\mu} \in \check{\Lambda}_{T}}{\oplus} V_{\breve{\mu}}
$$

according to the action of $T=Z\left(G_{\text {enh }}\right)$. Let us also note that by the definition of $G_{\text {enh }}$, the central character of the action of $G \hookrightarrow G_{\text {enh }}$ on each $V_{\breve{\mu}}$ equals $\left.\check{\mu}\right|_{Z(G)}$. Now $\operatorname{Rep}\left(\overline{G_{\text {enh }}}\right)$ is defined to consist of all $V \in \operatorname{Rep}\left(G_{\mathrm{enh}}\right)$ with the following property: for every $\check{\mu}$, the weights of $V_{\check{\mu}}$, regarded as a $G$-representation, are $\leq \check{\mu}$.

Note that the set of weights of a given $G$-representation is invariantly defined, i.e., independent of the choice of the Cartan or Borel subgroups.

D.2.2. L. Renner proved that the affine scheme $\overline{G_{\text {enh }}}$ is of finite type and normal (this follows from [Re, Thm 5.4 (a)]). In the characteristic zero case this is also proved in Vi].

\footnotetext{
${ }^{13}$ It was defined and studied by E. Vinberg Vi in characteristic 0 and then by A.Rittatore in arbitrary characteristic, see Ri1, Ri2, Ri3 Ri4].
} 
D.2.3. The Tannakian formalism provides the following description of the $S$-points of $\overline{G_{\text {enh }}}$ for any $k$-scheme $S$ : an element of the monoid $\operatorname{Maps}\left(S, \overline{G_{\mathrm{enh}}}\right)$ is a collection of assigments

$$
V \in \operatorname{Rep}\left(\overline{G_{\mathrm{enh}}}\right) \quad \rightsquigarrow \quad g_{V} \in \operatorname{End}_{\mathcal{O}_{S}}\left(V \otimes \mathcal{O}_{S}\right),
$$

compatible with maps $V_{1} \rightarrow V_{2}$ in $\operatorname{Rep}\left(\overline{G_{\text {enh }}}\right)$ and such that

$$
g_{V_{1} \otimes V_{2}}=g_{V_{1}} \otimes g_{V_{2}} .
$$

\section{D.3. The homomorphism $\bar{\pi}: \overline{G_{\text {enh }}} \rightarrow \overline{T_{\text {adj }}}$.}

D.3.1. The algebraic monoid $\overline{T_{\text {adj }}}$. As before, set $T_{\text {adj }}:=T / Z(G)$. Let $I$ denote the set of vertices of the Dynkin diagram of $G$. The simple root corresponding to $i \in I$ is a homomorphism $T_{\text {adj }} \rightarrow \mathbb{G}_{m}$. These homorphisms define a canonical isomorphism

$$
T_{\mathrm{adj}} \stackrel{\sim}{\longrightarrow} \prod_{i \in I} \mathbb{G}_{m}
$$

The inverse isomorphism is given by the fundamental coweights $\mathbb{G}_{m} \rightarrow T_{\text {adj }}$.

Let $\overline{T_{\text {adj }}}$ denote the algebraic monoid $\prod_{i \in I} \mathbb{A}^{1}$. The open embedding $\mathbb{G}_{m} \hookrightarrow \mathbb{A}^{1}$ induces a canonical open embedding

$$
T_{\text {adj }} \hookrightarrow \overline{T_{\text {adj }}} .
$$

D.3.2. The homomorphism $\bar{\pi}$. Define a homomorphism of algebraic groups $\pi: G_{\text {enh }} \rightarrow T_{\text {adj }}$ to be the composition

$$
G_{\text {enh }}:=(G \times T) / Z(G) \rightarrow T / Z(G)=T_{\text {adj }} .
$$

The functor $\operatorname{Rep}\left(T_{\text {adj }}\right) \rightarrow \operatorname{Rep}\left(G_{\text {enh }}\right)$ corresponding to $\pi$ maps $\operatorname{Rep}\left(\overline{T_{\text {adj }}}\right)$ to $\operatorname{Rep}\left(\overline{G_{\text {enh }}}\right)$. So $\pi$ extends to a homomorphism of algebraic monoids

$$
\bar{\pi}: \overline{G_{\text {enh }}} \rightarrow \overline{T_{\text {adj }}} .
$$

Note that

$$
\bar{\pi}^{-1}\left(T_{\mathrm{adj}}\right)=G_{\mathrm{enh}} .
$$

This follows from the fact that for every $V \in \operatorname{Rep}\left(G_{\text {enh }}\right)$ there exists a character $\chi: T_{\text {adj }} \rightarrow \mathbb{G}_{m}$ such that $V \otimes(\chi \circ \pi) \in \operatorname{Rep}\left(\overline{G_{\text {enh }}}\right)$ (here $\chi \circ \pi$ is considered as a 1-dimensional representation of $\left.G_{\text {enh }}\right)$.

D.4. The non-degenerate locus of Vinberg's semi-group. In this subsection we recall some facts whose proofs can be found in [Vi, Sect. 8] in the characteristic zero case and in [Ri1, Ri3] in general (there are also some hints in [Bo, Sect. 1.1]).

D.4.1. Recall that $G_{\mathrm{enh}}:=(G \times T) / Z(G)$, so a weight of $G_{\mathrm{enh}}$ is a pair

$$
\left(\check{\lambda}_{1}, \check{\lambda}_{2}\right) \in \check{\Lambda}_{G} \times \check{\Lambda}_{T}
$$

such that $\check{\lambda}_{1}-\check{\lambda}_{2}$ belongs to the root lattice. For each dominant $\check{\lambda} \in \Lambda_{G}$ let $V_{\check{\lambda}, \check{\lambda}} \in \operatorname{Rep}\left(\overline{G_{\text {enh }}}\right)$ denote the irreducible representation with highest weight $(\check{\lambda}, \check{\lambda})$.

Given a point $g \in G_{\mathrm{enh}}(k)$, let $A_{g}$ denote the set of dominant weights $\check{\lambda} \in \Lambda_{G}$ such that the action of $g$ on $V_{\check{\lambda}, \check{\lambda}}$ is non-zero.

The following fact is well-known:

Lemma D.4.2. The following properties of $g \in G_{\text {enh }}$ are equivalent:

(i) $A_{g}$ is the set of all dominant weights of $G$;

(ii) $A_{g}$ is not contained in any wall of the dominant cone of $\check{\Lambda}_{G} \otimes \mathbb{Q}$. 
It is clear that the set of elements of $G_{\text {enh }}(k)$ with property (ii) corresponds to an open subscheme of $\overline{G_{\text {enh }}}$; we denote it by $\frac{\circ}{G_{\text {enh }}}$.

Definition D.4.3. The subscheme $\frac{\circ}{G_{\mathrm{enh}}} \subset \overline{G_{\mathrm{enh}}}$ is called the non-degenerate locus of $\overline{G_{\mathrm{enh}}}$.

D.4.4. An element $\left(g_{1}, g_{2}\right) \in G_{\text {enh }} \times G_{\text {enh }}$ defines a regular map $\overline{G_{\text {enh }}} \rightarrow \overline{G_{\text {enh }}}$ given by

$$
x \mapsto g_{1} \cdot x \cdot g_{2}^{-1} .
$$

Thus $G_{\text {enh }} \times G_{\text {enh }}$ acts on the variety $\overline{G_{\text {enh }}}$. The open subscheme $\overline{G_{\text {enh }}} \subset \overline{G_{\text {enh }}}$ is clearly preserved under this action.

D.4.5. It is known that the scheme $\frac{\circ}{G_{\text {enh }}}$ is smooth and moreover, the restriction of the morphism $\bar{\pi}: \overline{G_{\text {enh }}} \rightarrow \overline{T_{\text {adj }}}$ to $\overline{G_{\text {enh }}}$ is smooth.

It is also known that the action of $G \times G$ on $\overline{G_{\text {enh }}}$ is transtivite relative to $\overline{T_{\text {adj }}}$, in the sense that the action map

$$
(G \times G) \times \frac{\circ}{G_{\text {enh }}} \rightarrow \frac{\circ}{G_{\text {enh }}} \times \frac{\circ}{T_{\text {adj }}} \frac{\circ}{G_{\text {enh }}}
$$

is surjective and flat. Moreover, it is known that the morphism (D.3) is smooth.

D.4.6. Consider $(G \times G) \times \frac{\circ}{G_{\text {enh }}}$ as a group-scheme over $\frac{\circ}{G_{\text {enh }}}$. Define a closed group-subscheme $\operatorname{Stab}_{G \times G} \subset(G \times G) \times \frac{\circ}{G_{\text {enh }}}$ to be the pre-image of the diagonal $\frac{\circ}{G_{\text {enh }}} \hookrightarrow \frac{\circ}{G_{\text {enh }}} \frac{\times}{T_{\text {adj }}} \frac{\circ}{G_{\text {enh }}}$ with respect to the map (D.3). The fiber of $\operatorname{Stab}_{G \times G}$ over $x \in \overline{G_{\text {enh }}}(k)$ is the stabilizer of $x$ in $G \times G$; it will be denoted by $\operatorname{Stab}_{G \times G}(x)$.

It follows from Sect. D.4.5 that the scheme $\operatorname{Stab}_{G \times G}$ is smooth over $\frac{\circ}{G_{\text {enh }}}$.

Remark D.4.7. The torus $T=Z\left(G_{\mathrm{enh}}\right)$ acts on $\overline{G_{\mathrm{enh}}}$ by translations. It is easy to show that the resulting action of $T$ on $\frac{0}{G_{\mathrm{enh}}}$ is free. 14 It is also easy to show that the quotient $\frac{\circ}{G_{\mathrm{enh}}} / T$ depends only on $G_{\mathrm{adj}}:=G / Z(G)$.

It is well known that this quotient is projective and smooth; this is the wonderful compactification 15 of $G_{\text {adj }}:=G / Z(G)$.

It is clear that the subscheme $\operatorname{Stab}_{G \times G}(x) \subset G \times G$ corresponding to $x \in \overline{G_{\text {enh }}}(k)$ depends only on the image of $x$ in $\frac{\circ}{G_{\text {enh }}} / T$.

\footnotetext{
${ }^{14}$ Let us note that for most groups $G$ (e.g., for $\left.G=S L(3)\right)$ the set of points of $\overline{G_{\text {enh }}}$ with trivial stablizer in $T$ is larger than $\frac{\circ}{G_{\mathrm{enh}}}$.

${ }^{15}$ In characteristic 0 the wonderful compactification was defined in $\mathrm{DCP}$ (one of the equivalent definitions from $[\mathrm{DCP}$ is as follows: consider $\operatorname{diag}(\mathfrak{g}) \subset \mathfrak{g} \times \mathfrak{g}$ as a point of the Grassmannian of $\mathfrak{g} \times \mathfrak{g}$, then take the closure of its $(G \times G)$-orbit.) Without the characteristic zero assumption, the wonderful compactification was constructed in $\mathrm{Str}$ and then, in a different way, in $[\mathrm{BKu}$. An axiomatic definition of the wonderful compactification and a brief survey can be found in $[\mathrm{BP}$ Sect. 1].
} 
D.5. The section $\overline{\mathfrak{s}}: \overline{T_{\text {adj }}} \rightarrow \overline{G_{\text {enh }}}$. Now let us choose a Cartan subgroup $T_{\text {sub }} \subset G$ and a Borel $B \supset T_{\text {sub }}$. We will construct a section for the map $\bar{\pi}: \overline{G_{\text {enh }}} \rightarrow \overline{T_{\text {adj }}}$, which depends on these choices.

D.5.1. Let $\varphi: T \stackrel{\sim}{\longrightarrow} T_{\text {sub }}$ denote the isomorphism corresponding to the chosen Borel $B \supset$ $T_{\text {sub }}$. Define

$$
\mathfrak{s}: T_{\text {adj }}=T / Z(G) \rightarrow\left(T_{\text {sub }} \times T\right) / Z(G) \hookrightarrow(G \times T) / Z(G)=G_{\text {enh }}
$$

to be the homomorphism induced by the map

$$
T \rightarrow T_{\text {sub }} \times T, \quad \tau \mapsto\left(\varphi(\tau)^{-1}, \tau\right) .
$$

The composition $T_{\text {adj }} \stackrel{\mathfrak{s}}{\longrightarrow} G_{\text {enh }} \stackrel{\pi}{\longrightarrow} T_{\text {adj }}$ equals the identity.

\section{Lemma D.5.2.}

(i) The homomorphism $\mathfrak{s}: T_{\text {adj }} \rightarrow G_{\text {enh }}$ extends to a homomorphism of algebraic monoids

$$
\overline{\mathfrak{s}}: \overline{T_{\mathrm{adj}}} \rightarrow \overline{G_{\mathrm{enh}}} .
$$

(ii) $\overline{\mathfrak{s}}\left(\overline{T_{\text {adj }}}\right) \subset \overline{G_{\text {enh }}}$.

Proof. (i) It suffices to show that the functor $\operatorname{Rep}\left(G_{\text {enh }}\right) \rightarrow \operatorname{Rep}\left(T_{\text {adj }}\right)$ corresponding to the homomorphism $\mathfrak{s}: T_{\text {adj }} \rightarrow G_{\text {enh }}$ maps $\operatorname{Rep}\left(\overline{G_{\text {enh }}}\right)$ to $\operatorname{Rep}\left(\overline{T_{\text {adj }}}\right)$.

Set

$$
T_{\text {enh }}:=(T \times T) / Z(G) \stackrel{\varphi \times \text { id }}{\simeq}\left(T_{\text {sub }} \times T\right) / Z(G) \subset G_{\text {enh }} .
$$

The weights of $T_{\text {enh }}$ are pairs $\left(\check{\lambda}_{1}, \check{\lambda}_{2}\right) \in \check{\Lambda}_{T} \times \check{\Lambda}_{T}$, such that $\left.\lambda_{1}\right|_{Z(G)}=\left.\lambda_{2}\right|_{Z(G)}$.

Let $V \in \operatorname{Rep}\left(\overline{G_{\text {enh }}}\right)$. Then each weight $\left(\check{\lambda}_{1}, \check{\lambda}_{2}\right)$ of $T_{\text {enh }}$ on $V$ satisfies $\lambda_{1} \leq \check{\lambda}_{2}$ (by the definition of $\overline{G_{\text {enh }}}$ ).

By the definition of $\mathfrak{s}$, the weights of $T_{\text {adj }}$ on $V$ have the form $\check{\lambda}_{2}-\check{\lambda}_{1}$, where $\check{\lambda}_{1}$ and $\check{\lambda}_{2}$ are as above. So they are $\geq 0$. Therefore the action of $T_{\text {adj }}$ on $V$ extends to an action of $\overline{T_{\text {adj }}}$.

(ii) If $V=V_{\check{\lambda}, \check{\lambda}}$ then one of the weights of $\overline{T_{\text {adj }}}$ in $V$ equals $\check{\lambda}-\check{\lambda}=0$, so $\overline{T_{\text {adj }}}$ acts on this subspace by the identity endomorphism.

It is clear that the map $\overline{\mathfrak{s}}: \overline{T_{\text {adj }}} \rightarrow \overline{G_{\text {enh }}} \subset \overline{G_{\text {enh }}}$ defined in the lemma is a section for the projection $\bar{\pi}: \overline{G_{\text {enh }}} \rightarrow \overline{T_{\text {adj }}}$. This section depends on the choice of $T_{\text {sub }}$ and $B$.

D.5.3. Let $\operatorname{Stab}_{G \times G}(\overline{\mathfrak{s}})$ denote the $\overline{\mathfrak{s}}$-pullback of the subscheme $\operatorname{Stab}_{G \times G} \subset \bar{\circ} \frac{\circ}{G_{\text {enh }}} \times(G \times G)$ defined in Sect. D.4.6. Clearly $\operatorname{Stab}_{G \times G}(\overline{\mathfrak{s}})$ is a closed group-subscheme of $\overline{T_{\text {adj }}} \times(G \times G)$ viewed as a group-scheme over $\overline{T_{\text {adj }}}$.

Corollary D.5.4. $\operatorname{Stab}_{G \times G}(\overline{\mathfrak{s}})$ is smooth over $\overline{T_{\text {adj }}}$. The quotient

$$
\left(\overline{T_{\text {adj }}} \times(G \times G)\right) / \operatorname{Stab}_{G \times G}(\overline{\mathfrak{s}})
$$

exists and is isomorphic to $\overline{G_{\mathrm{enh}}}$ as a scheme over $\overline{T_{\mathrm{adj}}}$ equipped with an action of $G \times G$ and a section. In particular, the quotient is a quasi-affine scheme of finite type.

Proof. Follows from Sects. D.4.5 D.4.6.

D.6. Vinberg's semigroup and the "interpolating" group-scheme $\widetilde{G}$. 
D.6.1. Let $P, P^{-} \subset G$ be parabolics opposite to each other and $M:=P \cap P^{-}$. Let

$$
\gamma: \mathbb{G}_{m} \rightarrow Z(M)
$$

be a co-character dominant and regular with respect to $P$. Using these data we defined in Sect. 2.4.2 a closed group-subscheme $\widetilde{G} \subset \mathbb{A}^{1} \times G \times G$. On the other hand, in Sect. D.4.6 we defined a closed group-subscheme $\operatorname{Stab}_{G \times G} \subset(G \times G) \times \frac{\circ}{G_{\text {enh }}}=\overline{G_{\text {enh }}} \times(G \times G)$. We will show that $\widetilde{G}$ can, in fact, be obtained from $\operatorname{Stab}_{G \times G}$ by base change with respect to a certain map $\mathbb{A}^{1} \rightarrow \frac{\circ}{G_{\text {enh }}}$ (see Proposition D.6.4 below).

D.6.2. Choose a Cartan subgroup $T_{\text {sub }} \subset G$ contained in $M$; then $T_{\text {sub }} \supset Z(M)$. Choose a Borel $B \subset G$ so that $T_{\text {sub }} \subset B \subset P$. Then $T_{\text {sub }}$ identifies with $T$.

The composition

$$
\mathbb{G}_{m} \stackrel{\gamma}{\longrightarrow} Z(M) \hookrightarrow T_{\text {sub }} \stackrel{\sim}{\longrightarrow} T \rightarrow T_{\text {adj }}
$$

is a dominant co-character $\mathbb{G}_{m} \rightarrow T_{\text {adj }}$, so it extends to a homomorphism of algebraic monoids

$$
\mathbb{A}^{1} \rightarrow \overline{T_{\text {adj }}} .
$$

Composing (D.5) with the map $\overline{\mathfrak{s}}: \overline{T_{\text {adj }}} \rightarrow \frac{\circ}{G_{\text {enh }}}$ defined in Lemma D.5.2, we get a map

$$
\gamma^{\prime}: \mathbb{A}^{1} \rightarrow \overline{G_{\mathrm{enh}}}
$$

D.6.3. Let $\operatorname{Stab}_{G \times G}\left(\gamma^{\prime}\right)$ denote the $\gamma^{\prime}$-pullback of the subscheme $\operatorname{Stab}_{G \times G} \subset \frac{\circ}{G_{\text {enh }}} \times(G \times G)$ defined in Sect.D.4.6. Clearly $\operatorname{Stab}_{G \times G}\left(\gamma^{\prime}\right)$ is a closed group-subscheme of $\mathbb{A}^{1} \times(G \times G)$ viewed as a group-scheme over $\mathbb{A}^{1}$.

Proposition D.6.4. The group-subscheme $\widetilde{G} \subset \mathbb{A}^{1} \times(G \times G)$ defined in Sect. 2.4.2 is equal to $\operatorname{Stab}_{G \times G}\left(\gamma^{\prime}\right)$.

Proof. Both $\operatorname{Stab}_{G \times G}\left(\gamma^{\prime}\right)$ and $\widetilde{G}$ are closed group-subschemes of $\mathbb{A}^{1} \times(G \times G)$. Both are flat (and in fact, smooth) over $\mathbb{A}^{1}$ : for $\widetilde{G}$ this was proved in Proposition 2.3.8(i) and for $\operatorname{Stab}_{G \times G}\left(\gamma^{\prime}\right)$ this follows from smoothness of $\operatorname{Stab}_{G \times G}$ over $\frac{0}{G_{\text {enh }}}$ (see Sect. D.4.6). So it suffices to check that the two group-subschemes have the same fiber over any $t \in \mathbb{G}_{m}$.

By formula (2.11), the subscheme $\widetilde{G}_{t} \subset G \times G$ is defined by the equation

$$
\gamma(t) \cdot g_{1} \cdot \gamma(t)^{-1}=g_{2}, \quad g_{1}, g_{2} \in G .
$$

On the other hand, the fiber of $\operatorname{Stab}_{G \times G}\left(\gamma^{\prime}\right)$ over $t \in \mathbb{G}_{m}$ is the stabilizer of $\gamma^{\prime}(t) \in \overline{G_{\text {enh }}}$ with respect to the action of $(G \times G)$ on $\overline{G_{\text {enh }}}$ defined in Sect. D.4.4 so this fiber is defined by the equation

$$
g_{1} \cdot \gamma^{\prime}(t) \cdot g_{2}^{-1}=\gamma^{\prime}(t), \quad g_{1}, g_{2} \in G .
$$

By the definition of $\mathfrak{s}$ from Sect. D.5.1, $\gamma^{\prime}(t)$ differs from $\gamma(t)^{-1}$ only by an element of $Z\left(G_{\mathrm{enh}}\right)$. So (D.6) and (D.7) are equivalent .

Corollary D.6.5. The quotient $\left(\mathbb{A}^{1} \times G \times G\right) / \widetilde{G}$ exists and is a quasi-affine scheme of finite type over $\mathbb{A}^{1}$. 
Proof. By Proposition D.6.4 we can rewrite $\left(\mathbb{A}^{1} \times G \times G\right) / \widetilde{G}$ as

$$
\left(\mathbb{A}^{1} \times G \times G\right) / \operatorname{Stab}_{G \times G}\left(\gamma^{\prime}\right) .
$$

By Corollary D.5.4 the quotient (D.4) is a quasi-affine scheme of finite type over $\overline{T_{\text {adj }}}$. The quotient (D.8) is obtained from (D.4) by base change with respect to the map (D.5), so it is also quasi-affine.

\section{REFERENCES}

[AG] D. Arinkin and D. Gaitsgory, Singular support of coherent sheaves, and the geometric Langlands conjecture, Selecta Math. (N.S.) 21 (2015), no. 1, 1-199. Also: arXiv:math/1201.6343.

[BG] A. Braverman and D. Gaitsgory, Geometric Eisenstein Series, Invent. Math 150 (2002), 287-384. Also: arXiv:math/9912097

[BKa] R. Bezrukavnikov and D. Kazhdan, Geometry of second adjointness for $\mathfrak{p}$-adic groups, arXiv:math/1112.6340.

[BP] M. Brion and P. Polo, Large Schubert varieties, Represent. Theory 4 (2000), 97-126

$[\mathrm{BKu}] \mathrm{M}$. Brion and S. Kumar, Frobenius splitting methods in geometry and representation theory, Progress in Mathematics, 231. Birkhäuser, Boston, 2005.

[BN] I. Biswas and D. S. Nagaraj, Principal bundles over the projective line, J. Algebra 322 (2009), no. 10, 3478-3491.

[Bo] A. Bouthier, Dimension des fibres de Springer affines pour les groupes, Transformation Groups 20 (2015), no. 3, 615-663. Also: arXiv:math/1203.0975.

[Br] T. Braden, Hyperbolic localization of Intersection Cohomology, Transformation Groups 8 (2003), no. 3, 209-216. Also: arXiv:math/020225.

[DCP] C. De Concini and C. Procesi, Complete symmetric varieties. In: Invariant theory (Montecatini, 1982), 1-44, Lecture Notes in Math., 996, Springer, Berlin, 1983.

[Dr] V. Drinfeld, On algebraic spaces with an action of $\mathbb{G}_{m}$, arXiv:math/1308.2604, version 2.

[DrGa1] V. Drinfeld and D. Gaitsgory, On some finiteness questions for algebraic stacks, Geom. Funct. Anal. 23 (2013), no. 1, 149-294. Also: arXiv:math/1108.5351.

[DrGa2] V. Drinfeld and D. Gaitsgory, Compact generation of the category of D-modules on the stack of Gbundles on a curve, Cambridge Journal of Mathematics 3 (2015), no. 1-2, 19-125.

[DrGa3] V. Drinfeld and D. Gaitsgory, On a theorem of Braden, Transformation Groups 19 (2014), no. 2, 313-358. Also: arXiv:math/1308.3786.

[Ga] D. Gaitsgory, A "strange" functional equation for Eisenstein series and miraculous duality on the moduli stack of bundles, arXiv:math/1404.6780.

[Gi] V. Ginzburg, Induction and restriction of character sheaves, I. M. Gel'fand Seminar, 149-167, Adv. Soviet Math. 16, Part 1, Amer. Math. Soc., Providence, RI, 1993.

[GR] D. Gaitsgory and N. Rozenblyum, DG indschemes, In: Perspectives in representation theory, 139-251, Contemp. Math., 610, Amer. Math. Soc., Providence, RI, 2014. Also arXiv:math/1108.1738.

[Kn] D. Knutson, Algebraic spaces. Lecture Notes in Math. 203, Springer-Verlag, Berlin-New York, 1971.

[KKMS] G. Kempf, F. F. Knudsen, D. Mumford, and B. Saint-Donat, Toroidal embeddings. I, Lecture Notes in Math. 339, Springer-Verlag, Berlin-New York, 1973.

[LM] G. Laumon and L. Moret-Bailly, Champs algébriques, Ergebnisse der Mathematik und ihrer Grenzgebiete (3 Folge, A Series of Modern Surveys in Mathematics), 39, Springer-Verlag, Berlin, 2000.

[Lur] J. Lurie, Higher Topos Theory, Annals of Mathematics Studies, 170, Princeton University Press, Princeton, NJ, 2009.

[Pu] M. Putcha, Linear algebraic monoids, London Mathematical Society Lecture Note Series, 133. Cambridge University Press, Cambridge, 1988

[Re] L Renner, Linear algebraic monoids. Encyclopaedia of Mathematical Sciences, 134. Invariant Theory and Algebraic Transformation Groups, V. Springer-Verlag, Berlin, 2005.

[Ri1] A. Rittatore, Monoides Algébriques et plongements des groupes, Thèse de Doctorat, Institut Fourier, 1997, available at http://www-fourier.ujf-grenoble.fr/THESE/pdf/t122.pdf

[Ri2] A. Rittatore, Algebraic monoids and group embeddings, Transform. Groups 3 (1998), no.4, 375-396.

[Ri3] A. Rittatore, Very flat reductive monoids, Publ. Mat. Urug. 9 (2001), no.4, 93-121.

[Ri4] A. Rittatore, Algebraic monoids with affine unit group are affine, Transform. Groups 12 (2007), no.3, 601-605.

[Str] E. Strickland, A vanishing theorem for group compactifications, Math. Ann. 277 (1987), no. 1, $165-171$. 
[Ti] D. Timashev, Homogeneous spaces and equivariant embeddings, Encyclopaedia of Mathematical Sciences, 138. Invariant Theory and Algebraic Transformation Groups, 8. Springer, Heidelberg, 2011.

[Vi] E. B. Vinberg, On reductive algebraic semigroups, E. B. Dynkin's Seminar, 145-182, Amer. Math. Soc. Transl. Ser. 2, 169, Amer. Math. Soc., Providence, RI, 1995. 